\title{
TEST-ReteSt RePEATABILITY OF MYOCARDIAL BLOOD FLOW MEASUREMENTS USING RUBIDIUM-82 POSITRON EMISSION TOMOGRAPHY
}

\author{
by \\ Matthew Efseaff, B.A. \\ A Thesis Submitted to \\ the Faculty of Graduate and Postdoctoral Affairs \\ in Partial Fulfillment of \\ the Requirements for the Degree of \\ Master of Science \\ Ottawa-Carleton Institute for Physics \\ Department of Physics, Carleton University \\ Ottawa, Ontario, Canada \\ Submitted July, 2012
}

Copyright (C) 2012

Matthew Efseaff 
Library and Archives

Canada

Published Heritage

Branch

395 Wellington Street

Ottawa ON K1A ON4

Canada
Bibliothèque et

Archives Canada

Direction du

Patrimoine de l'édition

395 , rue Wellington

Ottawa ON K1A ON4

Canada
Your file Votre référence

ISBN: 978-0-494-93568-2

Our file Notre référence

ISBN: $978-0-494-93568-2$
NOTICE:

The author has granted a nonexclusive license allowing Library and Archives Canada to reproduce, publish, archive, preserve, conserve, communicate to the public by telecommunication or on the Internet, loan, distrbute and sell theses worldwide, for commercial or noncommercial purposes, in microform, paper, electronic and/or any other formats.

The author retains copyright ownership and moral rights in this thesis. Neither the thesis nor substantial extracts from it may be printed or otherwise reproduced without the author's permission.
AVIS:

L'auteur a accordé une licence non exclusive permettant à la Bibliothèque et Archives Canada de reproduire, publier, archiver, sauvegarder, conserver, transmettre au public par télécommunication ou par l'Internet, prêter, distribuer et vendre des thèses partout dans le monde, à des fins commerciales ou autres, sur support microforme, papier, électronique et/ou autres formats.

L'auteur conserve la propriété du droit d'auteur et des droits moraux qui protege cette thèse. $\mathrm{Ni}$ la thèse ni des extraits substantiels de celle-ci ne doivent être imprimés ou autrement reproduits sans son autorisation.
In compliance with the Canadian Privacy Act some supporting forms may have been removed from this thesis.

While these forms may be included in the document page count, their removal does not represent any loss of content from the thesis.
Conformément à la loi canadienne sur la protection de la vie privée, quelques formulaires secondaires ont été enlevés de cette thèse.

Bien que ces formulaires aient inclus dans la pagination, il n'y aura aucun contenu manquant. 


\section{Abstract}

Rubidium-82 positron emission tomography (PET) imaging has been proposed for routine myocardial blood flow (MBF) quantification. Few studies have investigated the test-retest repeatability of this method. Same-day repeatability of rest MBF imaging was optimized with a highly automated analysis program using image-derived input functions and a dual spillover correction (SOC). The effects of heterogeneous tracer infusion profiles and subject hemodynamics on test-retest repeatability were investigated at rest and during hyperemic stress. Factors affecting rest MBF repeatability included gender, suspected coronary artery disease, and dual SOC ( $p<$ 0.001 ). The best repeatability coefficient for same-day rest $\mathrm{MBF}$ was $0.20 \mathrm{~mL} / \mathrm{min} / \mathrm{g}$ using a six-minute scan-time, iterative reconstruction, dual SOC, resting rate-pressureproduct (RPP) adjustment, and a left atrium image-derived input function. The serial study repeatabilities of the optimized protocol in subjects with homogeneous RPPs and tracer infusion profiles was 0.19 and $0.53 \mathrm{~mL} / \mathrm{min} / \mathrm{g}$ at rest and stress, and 0.95 for stress / rest myocardial flow reserve (MFR). Subjects with heterogeneous tracer infusion profiles and hemodynamic conditions had significantly less repeatable MBF measurements at rest, stress, and stress/rest flow reserve $(p<0.05)$. 


\section{Acknowledgements}

I express my sincere gratitude to my supervisor, Dr. Robert deKemp, for his support and guidance during my time at the University of Ottawa Heart Institute. He provided answers when I never would have found them and encouraged me to search for, and discover, the ones I could find on my own.

Many thanks to my wife, Janet, for her patience, unquestioned support, and for listening as I tried to explain concepts I did not fully understand.

I would like to thank May Aung and Kimberly Gardner in the PET lab for their assistance with reconstruction, Judy Etele for assistance with patient data, and my co-students in the research lab who answered questions and provided much-needed breaks from research. Special thanks to Ran Klein for assistance in implementing the methods and to both Ran and Jennifer Renaud for technical assistance with the FlowQuant program. 


\section{Statement of Originality}

There were two main components to this research. In the first component, test-retest repeatability of same-day rest myocardial blood flow, I optimized the image-derived input function methods, processed the subject images, and analyzed the data. This research resulted in a multi-authored manuscript, currently accepted for publication by the Journal of Nuclear Cardiology (1). Portions of this research were presented as a poster at the 2012 Society of Nuclear Medicine Annual Meeting, published as an abstract (2), and presented orally at the 2012 Molecular Function and Imaging Symposium. For the manuscript I was the lead author, wrote the first draft of the manuscript, and completed the revision process with the Journal editor. I created the poster and the slides for the presentation. Co-authors designed the repeatability study and participated in the revisions of the manuscript and poster.

In the second component of this research, test-retest repeatability of serial myocardial blood flow at rest, stress and of flow reserve, I processed the subject images and analyzed the resulting data. Portions of this research were presented as a poster and published as an abstract at the 2012 Canadian Association of Nuclear Medicine and Society of Nuclear Medicine Eastern Great-Lakes Annual Meeting, for which I wrote the abstract and created the poster. 


\section{Table of Contents}

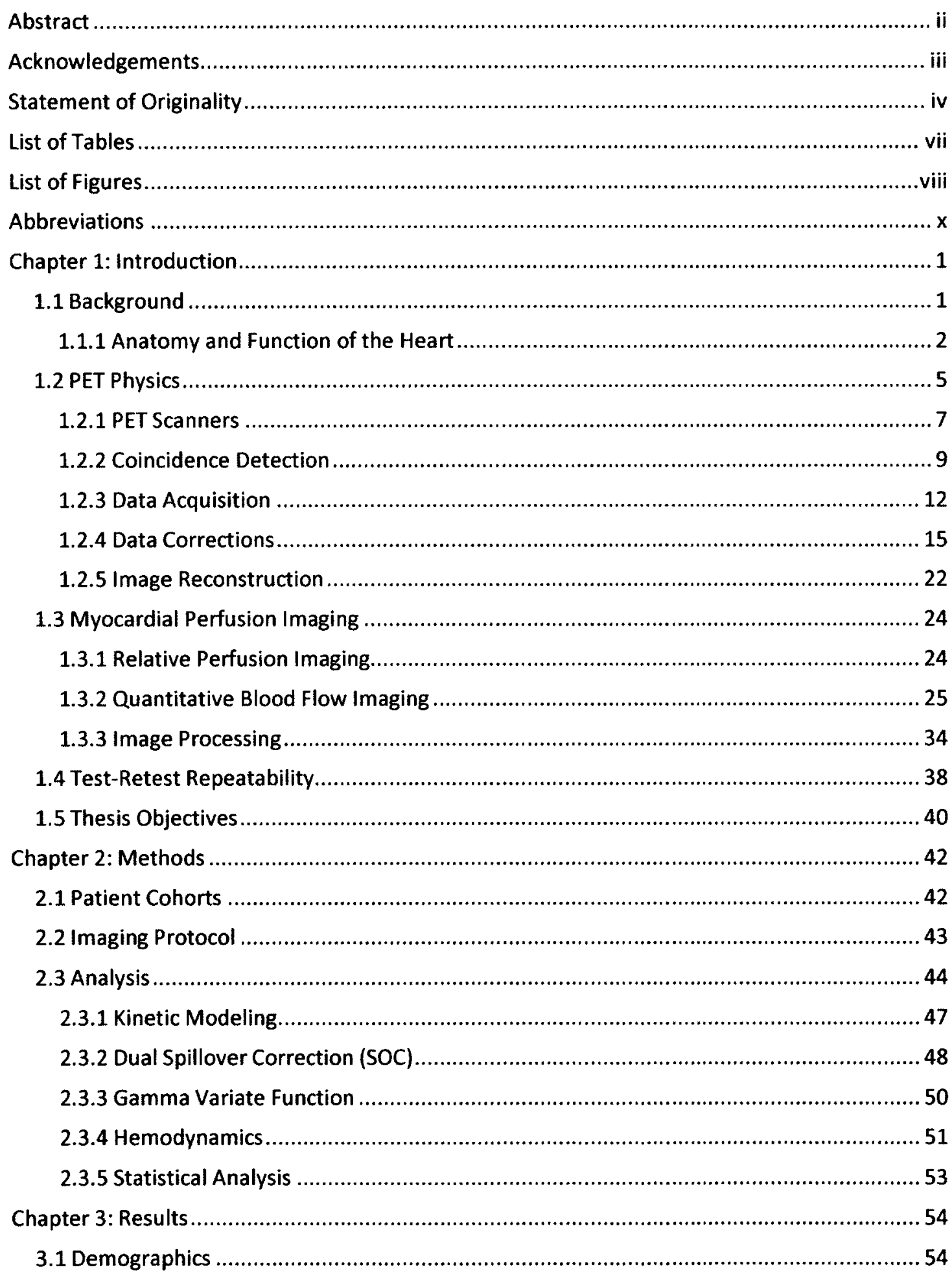




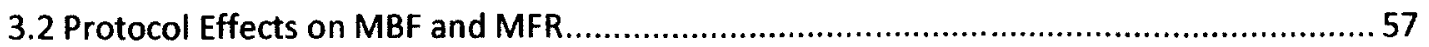

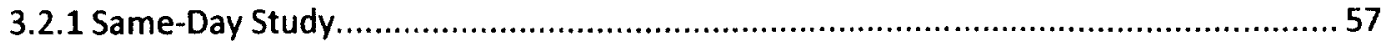

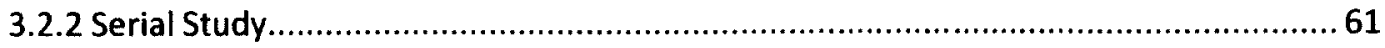

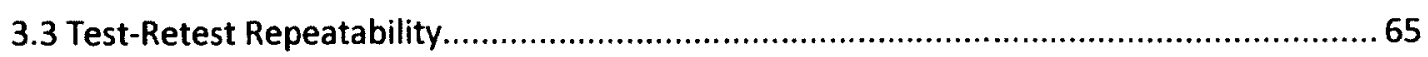

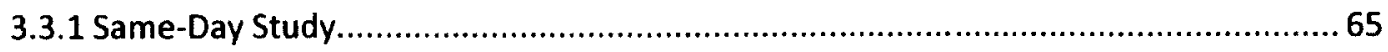

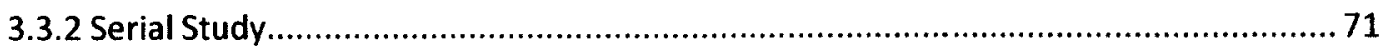

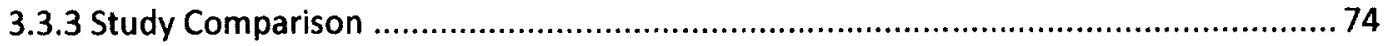

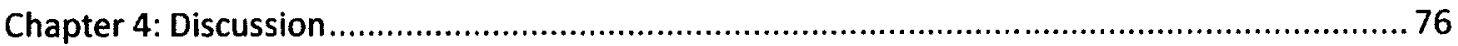

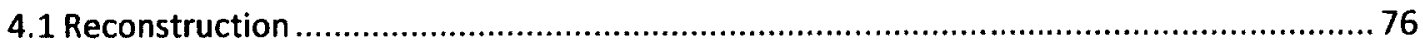

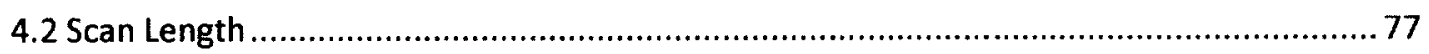

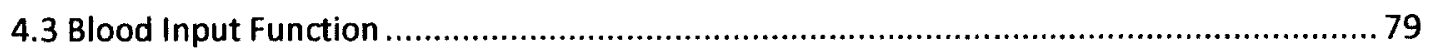

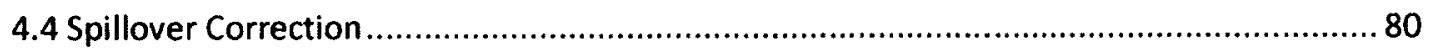

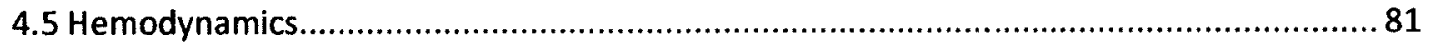

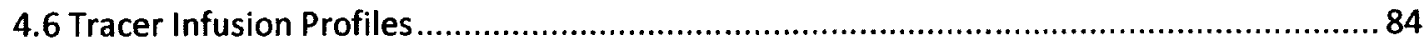

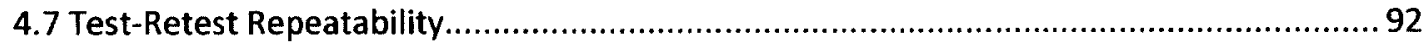

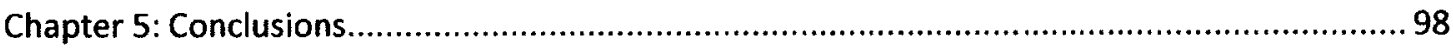

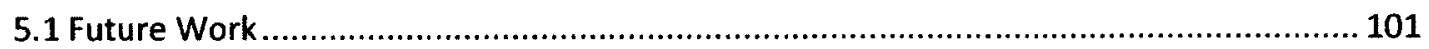

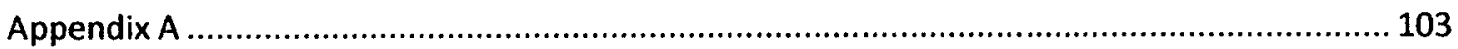

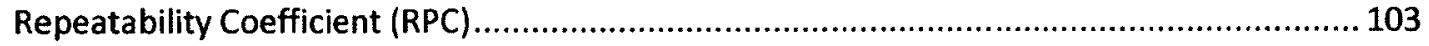

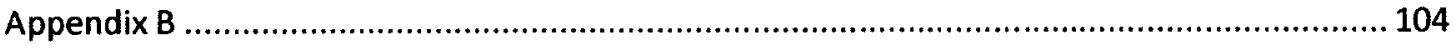

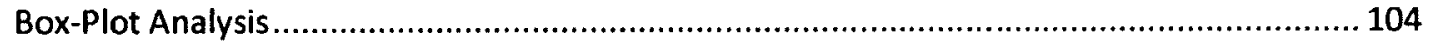

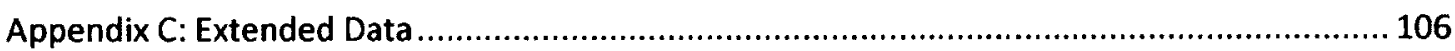

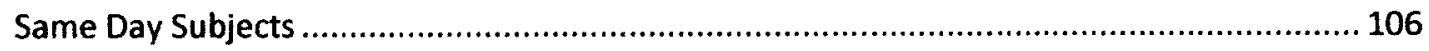

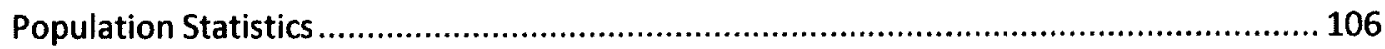

Left Ventricle ROI Arterial Input Function - Rest and Stress MBF ................................... 107

Left Atrium ROI Arterial Input Function - Rest and Stress MBF ....................................... 108

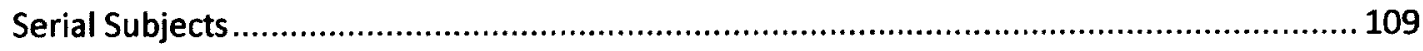

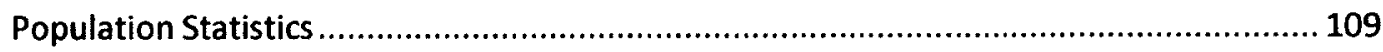

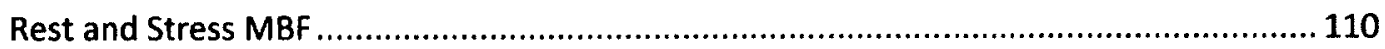

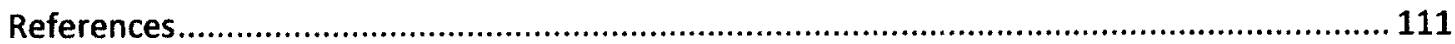




\section{List of Tables}

Table 1.1: Characteristics of commonly used PET MBF tracers...................................32

Table 3.1: Same-day study subject demographics.............................................55

Table 3.2: Serial study subject demographics.....................................................55

Table 3.3: Same-day study subject hemodynamics................................................56

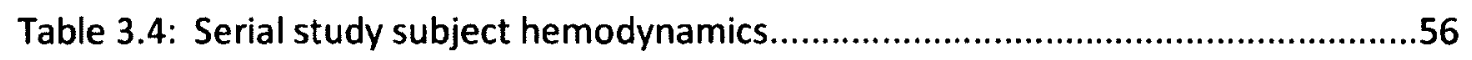

Table 3.5: Same-day rest MBF values with and without RPP-adjustment....................57

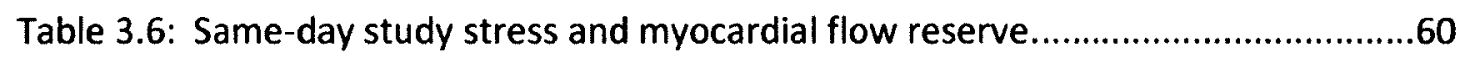

Table 3.7: Same-day study test-retest repeatability of rest MBF measurements..........66

Table 3.8: Same-day study test-retest repeatability of myocardial flow reserve............70

Table 3.9: Test-retest repeatability at rest and stress MBF, and MFR for different infusion profile and hemodynamic exclusion criteria.....................................72

Table 4.1: Test-retest repeatability of rest MBF compared to published literature........92

Table 4.2: Test-retest repeatability of stress MBF compared to published literature.....93

Table 4.3: Test-retest repeatability of myocardial flow reserve (MFR) compared to published literature. 


\section{List of Figures}

Figure 1.1: A cross-section through the four chambers of the human myocardium.........3

Figure 1.2: Radioactive positron $\left(\beta^{+}\right)$emission from an unstable nucleus.......................7

Figure 1.3: Illustration of the four coincidence event types in positron emission tomography: true (1), scattered (2), random (3), and cascade photon (4)..........10

Figure 1.4: Two-dimensional PET imaging (left) produces one sinogram per detector ring with radial $(R)$, angular $(\Theta)$, and axial $(Z)$ coordinates................................13

Figure 1.5: A time activity curve showing the amount of activity in absolute units of $\mathrm{kBq} / \mathrm{cm}^{3}$ in various regions in the heart over time .27

Figure 1.6: A generalized two-compartment tracer kinetic model showing a schematic of the dynamic rate-constant processes between spaces...............................27

Figure 1.7: A simplified illustration of $\mathrm{a}^{82} \mathrm{Sr} /{ }^{82} \mathrm{Rb}$ generator. 35

Figure 1.8: Cross-sectional planes of the myocardium and their corresponding PET images.

Figure 1.9: Three-dimensional mesh grid of the LV (left) represented in polar map

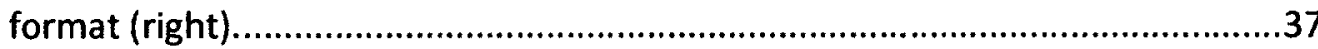

Figure 1.10: Time activity curves for the blood input function (red circles), measured myocardium (blue stars), and modeled myocardium (cyan).

Figure 1.11: Time activity curves of repeated rest scans performed 15 days apart........39

Figure 2.1: Horizontal and vertical long axis sample images showing the maximal extent of the left atrium region of interest.

Figure 2.2: A representative scan showing the tracer infusion profile parameters investigated.

Figure 3.1: Notched box-plot comparison of same-day scan $1 \mathrm{MBF}$ values between male and female subjects.

Figure 3.2: Notched box-plot comparison of infusion profile parameters extracted from the gamma variate function fit to the blood time-activity data. .63 
Figure 3.3: Hemodynamic parameter box-plot comparison of delta rate-pressureproduct at rest, stress and combined.

Figure 3.4: Correlation and Bland-Altman plots of test-retest differences in rest MBF values using the OSEM-6-SOC-RPP protocol with LV input.

Figure 3.5: Correlation and Bland-Altman plots of test-retest differences in rest MBF values using the OSEM-6-SOC protocol with LV input

Figure 3.6: Correlation and Bland-Altman plots of test-retest differences in rest MBF values using the OSEM-6-SOC-RPP protocol with LA input.

Figure 3.7: Correlation and Bland-Altman plots of test-retest differences in MFR values using the OSEM-6-LA-SOC-RPP protocol

Figure 3.8: Test-retest repeatability comparison between the same-day study and the serial study.....

Figure 4.1: Time activity curves showing similar blood curves, similar rest RPP values, and similar MBFs

Figure 4.2: Time activity curves showing similar blood curves, different rest RPP values, and different MBFs

Figure 4.3: An abnormal time activity curve (top) from a subject excluded at the 1.5 IQR level of the reduced Chi-squared goodness-of-fit parameter.

Figure 4.4: Heterogeneous time activity curves at stress from a subject excluded at the 1.5 IQR level of the mean transit-time parameter.

Figure 4.5: Resting time activity curves for the subject excluded at the 1.5 IQR exclusion level for the clearance-time parameter. .89

Figure 4.6: Time activity curves at stress for the same subject as in Fig. 4.5. .90

Figure 4.7: Distribution of rate-pressure-products box plot comparison between sameday and serial study groups.

Figure B.1: A notched box-plot comparison between two groups of data. 105 


\section{Abbreviations}

$\begin{array}{ll}\text { ATP } & \text { Adenosine TriPhosphate } \\ \text { ANOVA } & \text { ANalysis Of Variance } \\ \text { Bq } & \text { Becquerel (decays/s) } \\ \text { CAD } & \text { Coronary Artery Disease } \\ \text { CT } & \text { Computed Tomography } \\ \text { CV } & \text { Coefficient of Variation (100\% } \times \text { standard deviation / mean) } \\ \text { DV } & \text { Distribution Volume (mL/g) } \\ \text { FBP } & \text { Filtered BackProjection } \\ \text { FBV } & \text { Fractional Blood Volume (unit-less fraction) } \\ \text { FORE } & \text { FOurier REbinning } \\ \text { FOV } & \text { Field Of View } \\ \text { GV } & \text { Gamma Variate } \\ \text { IQR } & \text { InterQuartile Range } \\ \text { LA } & \text { Left Atrium } \\ \text { LAD } & \text { Left Anterior Descending } \\ \text { LCX } & \text { Left Circumflex Artery } \\ \text { LOR } & \text { Line Of Response } \\ \text { LV } & \text { Left Ventricle } \\ \text { MBF } & \text { Myocardial Blood Flow (mL/min/g) } \\ \text { MFR } & \text { Myocardial Flow Reserve (unit-less ratio: stress MBF/rest MBF) } \\ \text { MLEM } & \text { Maximum Likelihood-Expectation Maximization } \\ \text { MPI } & \text { Myocardial Perfusion Imaging } \\ \text { MTT } & \text { Mean Transit-Time (s) } \\ \text { MVD } & \text { Multi-Vessel Disease } \\ \text { OSEM } & \text { Ordered Subset Expectation Maximization } \\ \text { P } & \text { Probability, or confidence, that the null hypothesis is true } \\ \text { PET } & \text { Positron Emission Tomography } \\ \text { PMT } & \text { PhotoMultiplier Tube } \\ \text { PS } & \text { Permeability-Surface-area-product (mL/min/g) } \\ \text { PV } & \text { Partial Volume } \\ \text { RCA } & \text { Right Coronary Artery } \\ \text { ROI } & \text { Region Of Interest } \\ \text { RPC } & \text { RePeatability Coefficient } \\ \text { RPP } & \text { Rate-Pressure-Product (beats/min } \times \text { mm Hg) } \\ \text { SD } & \text { Standard Deviation } \\ \text { SOC } & \text { dual SpillOver Correction } \\ \text { Tc } & \text { Clearance Time (s) } \\ \text { TAC } & \text { Time-Activity Curve } \\ \text { UOHI } & \text { University of Ottawa Heart Institute } \\ & \end{array}$




\section{Chapter 1: Introduction}

\subsection{Background}

Positron emission tomography (PET) is one of many medical imaging modalities. It produces three-dimensional images of functional molecular processes in a subject as opposed to planar or structural anatomic imaging modalities such as $\mathrm{x}$-ray radiographs or computed tomography. This allows investigators and doctors to 'see' biological processes inside the living body, thus improving the understanding of disease processes and improve treatment for patients. Although PET imaging is non-invasive, a radioactive tracing agent must first be injected into the subject's bloodstream. The agent is taken up into a region or tissue of interest depending on the affinity of the tracer, and imaging reveals where the tracer resides by detecting the radioactive decay emissions.

Much research and labor has gone into the treatment and prevention of heart disease and heart attacks. Despite this, the Heart \& Stroke Foundation of Canada reports that heart disease is still one of the top three leading causes of death (3). The National Heart Lung and Blood Institute in the United States reports that coronary heart disease is the most common type of heart disease and is the primary cause of death in both men and women (4). Coronary artery disease (CAD) is a slowly advancing condition that progressively reduces the vital blood flow to the heart muscle (myocardium) through atherosclerosis. The costs associated with treating late-diagnosed heart disease and 
strokes exceeds twenty billion dollars annually in Canada alone (3). PET is one of the imaging modalities that has helped bring a decline in the prevalence of heart disease in recent years.

\subsubsection{Anatomy and Function of the Heart}

The human heart has two pumping stations: right atrium and ventricle, and left atrium (LA) and ventricle (LV), as shown in Figure 1.1. The right ventricle is responsible for pumping oxygen-depleted blood to the lungs for re-oxygenation while the right atrium acts as a holding tank for incoming blood while the ventricle contracts. Newly oxygenated blood from the lungs enters the LV through the LA reservoir and is pumped throughout the body. When a subject begins moderate to vigorous activity, the LV must increase its pumping action to deliver oxygen and nutrients to the areas in demand.

Three main arteries supply the LV myocardium with blood: the left anterior descending (LAD), the left circumflex (LCX), and the right coronary artery (RCA) (5). Many conditions, diseases, and situations are known to cause heart dysfunction. Principal among these is CAD, which causes atherosclerotic stenosis (vessel narrowing due to plaque accumulation) and may result in subjects experiencing ischemia and plaque rupture that lead to heart attacks. Microvascular disease, referring to disease in the small vessels leading from the main trunks throughout the LV, is also a major cause of clinical symptoms of heart disease. PET imaging with an appropriate radiotracer is able to 


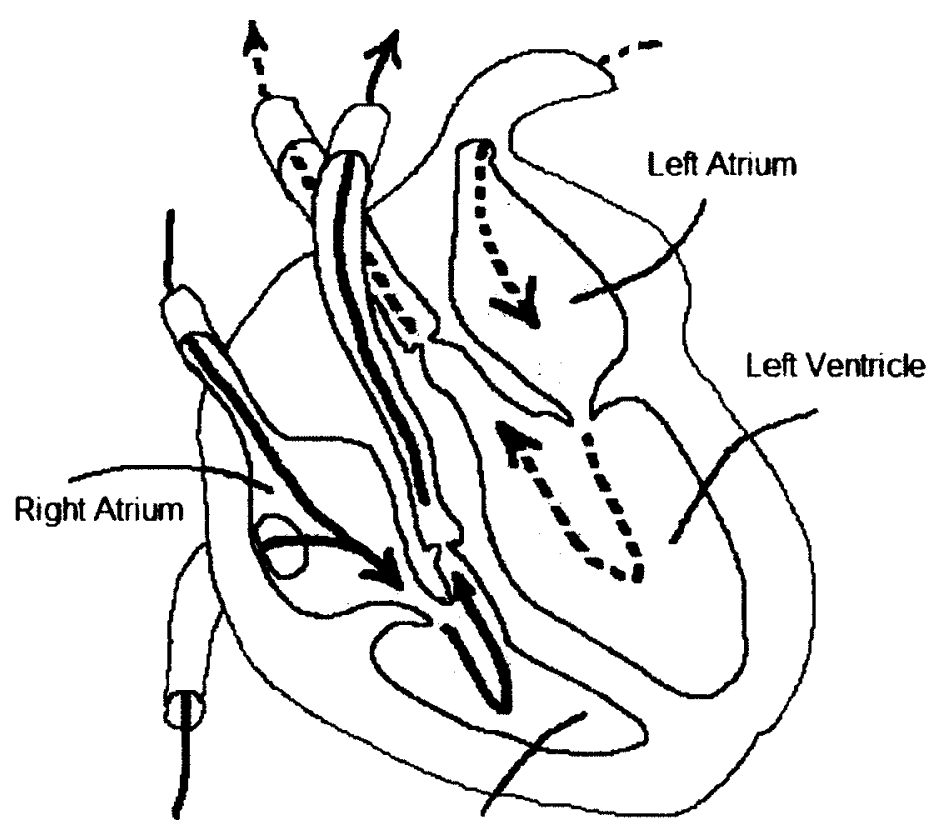

Right Ventricle

Figure 1.1: A cross-section through the four chambers of the human myocardium. Oxygen-depleted blood returns from the body (solid blue line) through the superior and inferior vena cava into the right atrium. The right atrium and ventricle pump blood to the lungs. Re-oxygenated blood (dashed red line) returns to the left side of the heart through pulmonary veins into the atrium and is pumped through the aorta to the body by the left ventricle.

investigate the condition of these arteries and micro-vessels through quantitative blood flow analysis.

Functional myocardial perfusion imaging (MPI) detects the amount of blood reaching various parts of the heart muscle. A partial blockage, or narrowing, in a major artery from the buildup of atherosclerotic plaque, called a stenosis, will reduce the amount 
blood and tracer perfusing that portion of the myocardium, and the corresponding image region will display reduced tracer uptake relative to the other areas of normal perfusion.

PET MPI is performed in two stages: during resting conditions and under hyperemic (increased blood flow) stress. The heart can be stressed by having the subject exercise, or put under stress-like conditions through pharmacological drug injection. During treadmill exercise the target heart rate is at least $85 \%$ of the subject's age-matched maximal heart rate. Pharmacological stress is an acceptable and equivalent alternative for subjects, or situations, in which physical exercise is unattainable or impossible (6). The ability of the heart to increase its blood volume output is also a good measure of heart health. Resting images of the myocardium are compared with images during stress conditions enabling physicians to determine the severity of disease and what type of medical intervention or therapy may be necessary.

A hemodynamic measurement of the myocardial blood flow demand is the ratepressure-product (RPP). This measurement is the heart rate multiplied by the systolic blood pressure and is a measure of cardiac work. As both heart rate and systolic blood pressure are subject specific and affected by various diseases, the RPP is an important physiological index used in quantitative myocardial blood flow imaging (MBF). The RPP is often used in subject studies to normalize resting population MBF measurements and has helped explain age-related increases in blood flow (7). 


\subsection{PET Physics}

The full complement of the physics principles behind PET are beyond the scope of this thesis. However, an introduction to the most important features of this useful imaging modality is presented, from atomic physics to biologic interactions.

PET imaging requires radioactive isotopes that emit positrons. Positron-emitting isotopes have an excess of protons in the nucleus. These nuclei follow one of two competing paths that allow them to achieve stability: electron capture and positron emission. Electron capture occurs when an inner shell electron is captured by a proton in the nucleus, converting the proton to a neutron and lowering the atomic number by one. This can take place if the rest state energy of the parent atom is larger than the rest energy of the daughter atom plus the binding energy of the captured electron. Heavier elements use the electron capture path to stabilize themselves much more frequently than lighter isotopes due to the higher likelihood that inner shell electrons will be close to the nucleus. Positron emission occurs when a proton ejects a positron $\left(\beta^{+}\right)$and transforms into a neutron. This can take place if the rest energy of the parent nucleus is larger than the rest mass of the daughter nucleus minus the rest mass of two electrons,

$$
M(A, Z)>M(A, Z-1)+2 m_{e}
$$

where $m_{e}$ is the rest mass of the electron, $A$ is the atomic mass, and $Z$ is the atomic 
number. The decay process is illustrated below,

$$
{ }_{Z}^{A} X \rightarrow{ }_{Z-1}^{A} Y+\beta^{+}+v_{e}
$$

where $X$ and $Y$ are the parent and daughter isotopes and $v_{e}$ is an electron neutrino. The emission energy of the positron is shared with the simultaneously emitted neutrino, resulting in a range of possible energies from zero to the maximum available.

The emitted positron will lose its kinetic energy through inelastic collisions with surrounding electrons and nuclei. When it comes to rest, or a very low kinetic energy, it will annihilate with a nearby antiparticle, the electron. In tissue this usually occurs within a nanosecond and a few millimeters (8). The process of annihilation transforms the rest energies of the two particles into electromagnetic energy, or photons. Figure 1.2 shows the process from nucleus to annihilation photon emission. Conservation of momentum requires that at least two photons are created. The creation of three photons is about 1000 times less likely (8). A two-photon event will create both with 511 keV of energy traveling in opposite directions. If the positron is not at rest when it annihilates with the electron, the photon system will retain some residual momentum. This results in a slight non-collinearity from $180^{\circ}$ of about $\pm 0.25^{\circ}$ (for a standard $80 \mathrm{~cm}$ PET scanner the effect on spatial resolution can be expressed as a full width at half maximum of up to two millimeters) (9). 


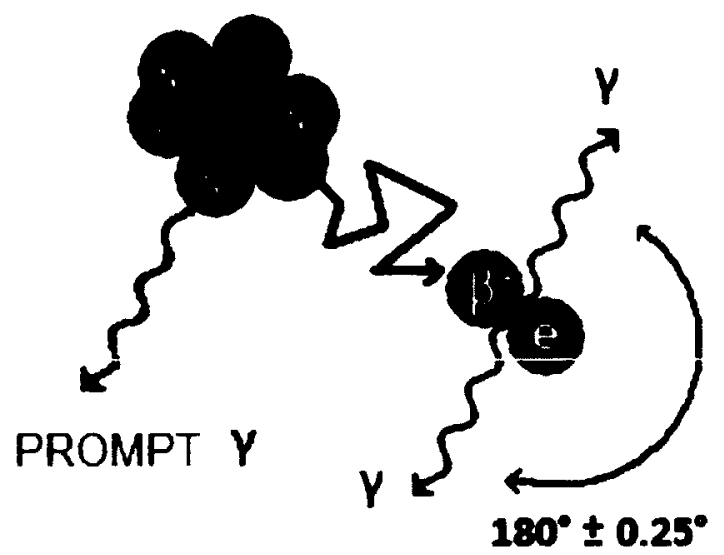

Figure 1.2: Radioactive positron $\left(\beta^{+}\right)$emission from an unstable nucleus. The positron loses energy in the surrounding medium and annihilates with an electron, creating two collinear $511 \mathrm{keV}$ photons ( $\gamma$ ). Depending on the isotope, there is also the possibility of producing prompt gammas with the positron decay.

Some isotopes have a probability to emit cascade, or prompt, gamma photons immediately following positron emission. The myocardial blood flow tracer used in this study, ${ }^{82} \mathrm{Rb}$, has a prompt gamma ray emission at $777 \mathrm{keV}$ that occurs in $14 \%$ of positron decays.

\subsubsection{PET Scanners}

PET scanners are designed to take advantage of the emitted photon collinearity. Current scanners have multiple rings of photon detectors arranged in an annulus that encircles 
the source of radiation. The scanners used to collect the data for the studies included in this thesis had 24 detector rings. The area bounded inside the rings defines the imaging field of view (FOV). If two detectors capture photons within a short period of time, it is assumed that the annihilation event, and therefore the positron-emitting isotope, decayed inside the volume of space between the two detectors. This process is called coincidence detection. The volume between the two detectors is the line of response (LOR).

All of the annihilation photons created in PET have the same energy, so detectors are chosen to work optimally at $511 \mathrm{keV}$. Three of the more common commercial human PET systems are made with either cerium-doped gadolinium oxyorthosilicate $\left(\mathrm{Gd}_{2} \mathrm{SiO}_{5}: \mathrm{Ce}\right)$, bismuth germanate $\left(\mathrm{Bi}_{4} \mathrm{Ge}_{3} \mathrm{O}_{12}\right)$, or cerium-doped lutetium oxyorthosilicate $\left(\mathrm{Lu}_{2} \mathrm{SiO}_{5}: \mathrm{Ce}\right)(10)(11)$. Photon detection occurs through the scintillation process. Annihilation photons enter the detecting crystal and produce energetic elections through ionization. The electrons return to their ground energy states and produce a cascade of visible photons. These flashes of visible light (e.g., fluorescent green at $480 \mathrm{~nm}$ for BGO) are amplified by photomultiplier tubes (PMT) which turn the light into an electronic signal. The size of the signal is proportional to the energy deposited in the detector, and is also position-weighted to establish in which crystal or individual detector the flash of light occurred. 
Each photon interaction inside the crystal detector takes a finite amount of time to be processed by the system electronics and recorded as a coincidence event. During this processing time the detector is unable to process further events and experiences 'dead time.' At high activity levels, the photons could cause a constant stream of interacting detector events called pulse pile-up. Pulse pile-up causes coincidence events to be mispositioned and the amount of activity to be underestimated.

\subsubsection{Coincidence Detection}

A time stamp is associated with each photon when it is detected. If two photons are detected within a given time interval $\tau$, the coincidence timing window, then they are assumed to be from the same annihilation event. The coincidence timing window is usually on the order of four to ten nanoseconds (12). Not all photons detected in this way necessarily originated along the associated LOR or are from the same annihilation event. Figure 1.3 shows the four types of prompt coincidences that are possible: true, random, scattered, and prompt gamma.

True events are annihilation photons that are unaltered from creation, and ideally reflect the true isotope activity distribution. Random coincidence events occur when two photons from separate annihilation events are captured by chance within the coincidence timing window. Scattered photons are from the same annihilation event, but at least one photon has interacted with surrounding material and changed direction so the recorded LOR does not accurately reflect where the event took place. There are 


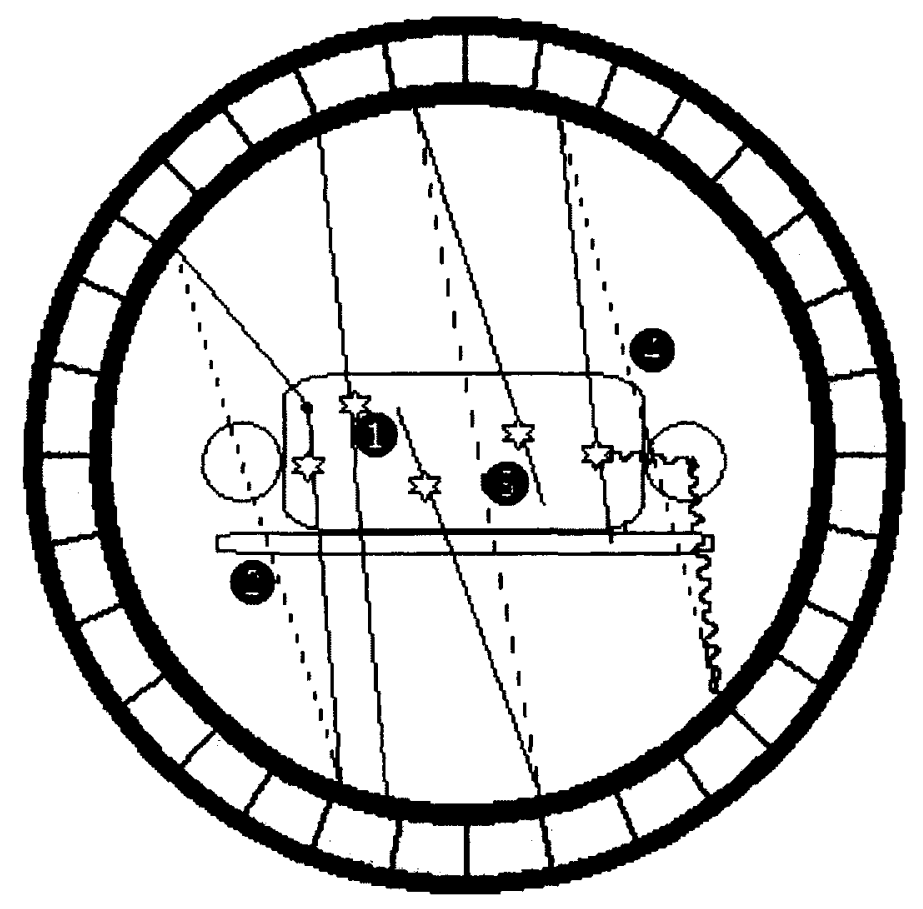

Figure 1.3: Illustration of the four coincidence event types in positron emission tomography: true (1), scattered (2), random (3), and cascade photon (4). Number four is a type of scattered event involving a 'prompt-gamma' photon. Annihilation photon paths are solid lines, prompt gamma rays are waved lines, and dashed lines correspond to the detected line of response.

six photon interactions possible: the photoelectric effect, Compton scattering, electronic and nuclear pair production, Rayleigh scattering, and photonuclear reactions.

Compton scattering has the highest probability of occurring over any other interaction mode due to its cross section at annihilation photon energies and the atomic number of the interacting medium (bone or soft-tissue). This occurs when a photon interacts with a 
loosely bound nuclear electron, ejecting the electron and altering the course of the photon. The energy of an annihilation photon after Compton scattering decreases according to the Compton equation:

$$
h \nu^{\prime}=\frac{h v}{1+\frac{h v}{m_{e} c^{2}}(1-\cos \theta)}
$$

where $h v^{\prime}$ is the energy of the deflected photon, $h v$ is the original photon energy, angle $\Theta$ is the deflection angle, and $m_{e} c^{2}$ is the rest energy of an electron. Larger angles of deflection impart more energy to the ejected election resulting in lower energy photons. Scattered photons that lose enough energy can be identified by energy discriminators in the detectors and discarded.

The fourth prompt coincidence type is from prompt gamma emissions. Following normal positron emission an excited nucleus will revert back to its ground energy state by emitting a gamma photon in a short time-frame $\left(<10^{-12}\right.$ seconds). Prompt gammas with energies inside the energy discrimination window, and higher energy prompt gammas that have lost energy through scatter, have the potential to form coincidence events with annihilation photons, thus introducing false LORs.

Annihilation photons are attenuated through Compton scatter and absorption, as noted above, as they exit the subject's body. The fraction of photon pairs that reach the detectors is described in the following equation as the total amount and type of matter 
they must traverse to reach the pair of coincidence detectors:

$$
F_{\text {detected }}=e^{-\mu x} e^{-\mu(T-x)}=e^{-\mu T}
$$

where $\mu$ is the linear attenuation coefficient of a certain type of matter, $T$ is the total thickness traversed, and $\mathrm{x}$ is the distance traversed by one photon. Attenuation correction is needed to correct for photon interactions in the subject's body so as to reflect the true radiotracer distribution.

\subsubsection{Data Acquisition}

Current PET scanners usually obtain data in 3-D mode, i.e., allowing photon events from all possible LORs to be paired between detector rings. However, the first scanners were designed with collimating septa that attenuated oblique angle LORs. This restricted the data accepted to 2-D rings or slices. Advantages included rejecting scattered photons and reducing the overall photon counting rate. This lowered the rate of random coincidences and minimized losses from detector dead-time. A large disadvantage to using septa was that a great number of useful photons from cross-ring planes were absorbed, thus limiting the total number of coincidence counts at the detectors.

When coincidence events are recorded, the data are binned into arrays indexed by spatial coordinates. The simplest case is 2-D imaging with a single ring of detectors, where each event LOR is associated with a radial $(R)$ and angular $(\theta)$ coordinate. These event arrays are often arranged as sinograms. The term sinogram refers to the shape a 
point source of activity will make when coincidence data are stored according to $R$ and $\theta$ coordinates. Figure 1.4 shows how the coincidence events in each detector ring form sinograms according to the three coordinates $(R, \theta, Z)$. The figure also shows how 3-D imaging introduces a fourth oblique angle coordinate, $\phi$ (recorded as the difference in rings), which creates many more sinograms compared to 2-D.
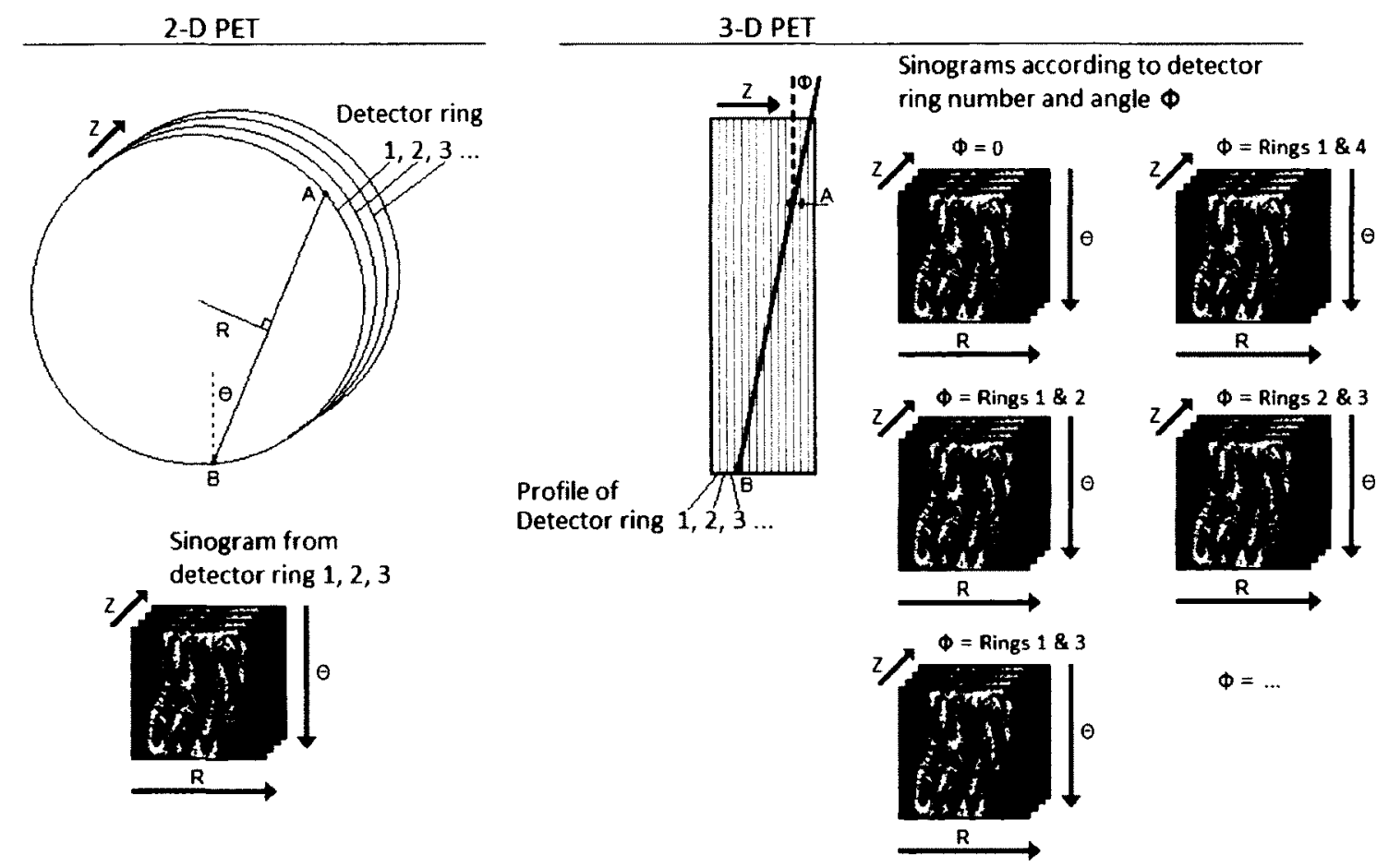

Figure 1.4: Two-dimensional PET imaging (left) produces one sinogram per detector ring with radial $(R)$, angular $(\theta)$, and axial $(Z)$ coordinates. Three-dimensional PET imaging (right) produces a sinogram for each detector ring as well as a sinogram for each possible oblique angle (Ф) as shown with a side view between points $[A]$ and $[B]$. 
Three-dimensional data acquisition has four- to eight-fold increased sensitivity compared to 2-D (9). In many cases this can result in less activity needing to be injected into the subject, which presents a lower radiation burden, rather than saturating the detectors with too many counts. At the same time, there is increased reliance on accurate scatter correction to produce results comparable to 2-D mode (13). At the University of Ottawa Heart Institute (UOHI) 3-D cardiac imaging is standard and this research study uses 3-D dynamic imaging exclusively.

Dynamic imaging measures the distribution and progression of tracer through a subject from the time of injection to a set end-point. This is possible due to the full ring of detectors encompassing the subject that acquire coincidence data by sampling the complete set of projection angles simultaneously. Coincidence data are sorted into variable length time-bins to capture the important temporal variations of the injected tracer. The standard dynamic heart scan at the UOHI consists of an eight- to ten-minute scan broken up into fifteen time-frames ( 9 frames $\times 10$ s, $3 \times 30,1 \times 60,1 \times 120,1 \times 240$ ).

An integrated hybrid PET and x-ray computed tomography (PET-CT) scanner is used at the UOHI to provide CT attenuation correction for the PET emission scan data. The CT system is a helical multi-slice $x$-ray scanner with a $4 \mathrm{~cm}$, 64-slice multiple-row detector array that is able to rapidly image the same FOV as the PET scan. Each slice has an axial thickness of $0.625 \mathrm{~mm}$. The patient couch translates through the FOV at a constant rate, or pitch, to acquire the full set of projections. 
In tomographic $x$-ray imaging a beam of poly-energetic photons is projected through a cross-sectional slice of a subject. A detector measures the intensity of transmitted photons that have not been absorbed or scattered by the subject. The distribution of energies in the beam are altered by scatter and absorption in the subject, so an effective beam energy is used. Effective energy is defined as the energy of a mono-energetic beam of photons that will produce the same intensity as a poly-energetic beam. The $x$ ray intensity measured at each detector is

$$
I_{d e t}=I_{O} e^{-\int_{0}^{d} \mu(x, \bar{E}) d x},
$$

where $I_{0}$ is the $x$-ray intensity without a subject in the FOV, $\mu$ is the linear attenuation coefficient in the subject, and $\bar{E}$ is the effective energy of the $x$-ray beam. Intensity projections in $\mathrm{CT}$ are acquired in either $180^{\circ}$ or $360^{\circ}$ rotations and reconstructed to obtain an image of linear attenuation. Single slice reconstruction is most commonly performed with filtered back projection (FBP), which will be discussed in the Image Reconstruction section.

\subsubsection{Data Corrections}

In order to obtain a set of dynamic images that ideally represent the true activity concentration in the body of a subject, several corrections must be made. Corrections can be performed prior to reconstruction while the data are still in projection format (sinograms) or included within the reconstruction process. Partial-volume correction, 
which depends on the size of the object being imaged and on the scanner resolution, will be discussed in section 1.3 as it is often estimated as part of the tracer kinetic modeling algorithms.

An important correction is for the rate of isotope decay. Isotope decay correction is applied to determine the amount of radiotracer present at the start of the scan given the amount of activity remaining at a particular frame time. The correction is as follows:

$$
A_{0}=A_{t} \times e^{\lambda t}
$$

where $A_{0}$ is the activity corrected back to time zero, $A_{t}$ is the measured activity at some time $(t)$, and lambda $(\lambda)$ is the decay constant. Lambda is the natural log of two divided by the half-life of the radiotracer.

\section{Detector Efficiency Normalization}

PET scanners are designed based on theoretical uniformity assuming that each detector has an identical efficiency, each PMT processes electronic signals identically, and detector block spacing is uniform. However, the actual physical characteristics of each detector element often vary enough to cause noticeable artifacts in reconstructed images. A direct, though simplistic, method for normalizing the number of counts is to expose every LOR with a uniform activity source (9). One technique is to rotate a rod source of germanium- $68\left({ }^{68} \mathrm{Ge}\right.$, half-life $=271$ days $)$ around the FOV. A normalization factor for each detector pair is then calculated by dividing the actual counts by the 
average counts of all detector pairs. After a subject PET scan, the counts at each LOR detector pair are divided by the unique normalization factor to obtain the normalized corrected counts (9).

The direct normalization method assumes extreme uniformity of activity concentration and similar scatter distribution as a subject scan. Differences in either parameter will result in bias and artifacts from poor normalizations. Normalization scans take many hours to complete in order to obtain the desired number of counts for low statistical variations. An improvement to detector efficiency normalization is a component-based correction. A component-based model measures average detector efficiency and geometric components and combines them into a correction matrix (15). To ensure the various correction factors remain accurate, different components need to be updated with quality assurance tests at various intervals and after scanner maintenance.

\section{Random Coincidences}

Random coincidences are a source of background signal in reconstructed images. Although this background is relatively uniform, it has the effect of adding bias and reducing image contrast around the organ of interest, which may make a medical diagnosis less certain. There are two main methods used to correct PET data for random coincidences: a delayed-window and calculation from detector singles rates (9). In the more common technique, the delayed-window, the number of coincidence events is sorted according to the time between events for two detectors. Inside the coincidence 
window the peak of the histogram will include both prompt and random events, but at a greater time difference between single photon events a 'delayed-coincidence-window' will include only random events. Subtraction of these random events occurs either in real-time, or the data are smoothed separately before subtraction to reduce statistical variations in the distribution of random events (14).

The second method involves measuring the number of unpaired or 'singles' events at each detector. The rate at which random coincidences occur for a given detector pair can be computed accurately by the following equation,

$$
R_{\text {random }}=\tau \times R_{\text {singles } A} \times R_{\text {singles } B},
$$

where $\tau$ is the coincidence timing window and $R_{\text {singles } A}$ and $R_{\text {singles } B}$ are the photon counting rates counted at the individual detectors. The total number of random events is subtracted from the total number of prompt coincidences, which also introduces increased statistical noise. It is therefore desirable to limit the rate of random coincidences, if possible, by using the smallest possible coincidence timing window. An important observation from Eq. 1.7 is that doubling the dose to the patient will double the true coincidence event rate, but will quadruple the random event rate (12).

Two scanners were used to acquire PET images for this research study. Before January 2010 all the subject scans were performed on a Discovery RX PET-VCT scanner (GE Healthcare, Waukesha, MI). This scanner used the delayed-window technique to correct for random coincidences. In January 2010 the UOHI installed a Discovery 690 PET-CT. 
This scanner uses singles counting rates at each detector to calculate a relatively noiseless correction for random coincidences.

\section{Scattered Coincidences}

After random coincidences are subtracted, the remaining counts are composed of true and scattered coincidences. Scattered event correction has been successfully achieved by several different approaches. Briefly, empirical methods involve plotting the number of photons detected versus photon energy and fitting the tails with either a 2 nd order polynomial or 1-D Gaussian. Multiple energy window techniques involve comparing either two or three adjacent windows about $511 \mathrm{keV}$ and then scaling and subtracting counts. Convolution approaches convolve projection image data with an experimentally determined scatter function and then subtract the result from the original projections. Completely analytic, or numeric, scatter simulation involves immense computing power and has taken too long in the past to be practical. However, if reasonable approximations are made, like eliminating multiple scatters on a single photon from the model, based on well understood physical principles, then accurate scatter correction can be successfully applied to image data (16).

Prompt coincidences in 3-D have a much higher scatter fraction (35-65\%) than 2-D (1020\%) and are an important effect to address (15). The UOHI systems use a model-based scatter correction that analytically calculates angular scatter distributions from the Klein-Nishina formula. The intensity of photons in the emission image and the CT- 
acquired attenuation image of the scattering medium are used, along with probability distributions from the Klein-Nishina formula to estimate the shape of the scatter distribution. The scale of these scatter projections are typically estimated by fitting to the count profiles, or 'scatter tails', outside the object defined as the scattering medium. When a uniform prompt-gamma correction is combined with scattered coincidence tailfitting outside the subject, the proper scale of the scatter correction is maintained (17).

\section{Attenuation}

Equation 1.4 demonstrated that the attenuation of annihilation photon pairs depended only on the total distance, and the spatial distribution, of attenuating material. This is a very important, and the largest magnitude, correction made to the data. Conservative estimates of the amount of attenuation by absorption and scatter, made in a $20 \mathrm{~cm}$ uniform water phantom, result in only $15 \%$ of photons reaching detectors. In a $40 \mathrm{~cm}$ phantom, similar to larger human subjects, only $1-2 \%$ of photon pairs are detected (13). Attenuation correction, therefore, is based on building a map of linear attenuation factors for every LOR. PET emission data are then multiplied by this attenuation map during reconstruction to obtain attenuation corrected images.

On dedicated PET scanners (without CT) this is carried out by completing a blank scan and a transmission scan. In the blank scan a positron-emitting source is rotated around the empty FOV. Then the transmission scan is completed in the same way with the subject in the FOV. Attenuation correction factors for each LOR are calculated simply by 
dividing the blank scan by the transmission scan counts. The attenuation correction factors for each LOR are then multiplied by the PET emission data to complete the weighting. This process is prone to subject movement as it takes between four and twenty minutes to complete each scan. Subject movement causes artifacts in the reconstructed image with attenuation correction. One improvement in this method uses up to three separate positron-emitting sources rotated about the FOV to speed up the process (15).

Attenuation correction at the UOHI is performed with high-speed, high-resolution, CT imaging. After transforming the linear attenuation coefficient map from $x$-ray energies to $511 \mathrm{keV}$, the PET emission data are weighted as described above. CT has the benefit of improved spatial resolution, and reduced statistical noise and scan speed (less than 2 seconds for a thorax scan) over PET transmission data. Minor drawbacks include nonlinear scaling of linear attenuation coefficients $(\mu)$ from the effective $x$-ray energy, typically 60 to $80 \mathrm{keV}(18)$, to $511 \mathrm{keV}$, and patient motion between scans, which is always an issue. Whereas PET emission data are acquired over several breathing and cardiac cycles, the CT scans can be performed at end- or mid-breath expiration.

\section{Dead-Time}

Detector dead-time arises from the finite time it takes for a photon to be processed by the electronic systems. An ideal system would have a linear response between the activity of a source in the FOV and the detected true coincidences. However, the 
number of detected events typically falls off with increased source activity, may begin to decrease at high activities, and can even fall to zero at extremely high activities depending on the system dead-time characteristics.

Two dead-time correction models are commonly used: paralyzable and non-paralyzable. In the paralyzable model the system will not accept any further input for a fixed time after activation. If a subsequent interaction starts before the end of the fixed time it will be ignored and a new time period is started. If photons impinge on a detector in rapid succession, only the first will be counted leading to an extended dead time. The nonparalyzable model only disregards subsequent events for a fixed amount of time. Further inputs do not restart the processing time period. This allows the maximum observable count rate to be a factor of at least $e(e \approx 2.718)$ higher than the paralyzable model (19). Many radiation detection systems are constructed with cascaded components subject to both paralyzable and non-paralyzable effects. Corrections to the data are made by applying scaling factors based on the dead-time model parameters, to estimate the true source activity.

\subsubsection{Image Reconstruction}

Following 3-D dynamic data acquisition and correction there is a mass of sinogram data that needs to be put into a format that allows for radiolabeled tracer localization in the subject's body. Fully 3-D reconstruction algorithms exist, but they require powerful computing and take much longer than 2-D methods. Rebinning algorithms, such as 
Fourier rebinning (FORE), reorder projections from 3-D data into approximate projections in 2-D. There are two types of reconstruction algorithms that take sinogram LOR projection data and transform them into an image: analytic and iterative.

Analytical reconstruction algorithms, primarily filtered backprojection (FBP), use simple and elegant transformations to produce an image. Ramp and low-pass filters are applied in the Fourier domain to the sinogram data and then inverse Fourier Transformed back into image space. An image matrix is defined and the number of counts in each LOR is linearly superimposed. This back-projection constructs the image analytically from each projection LOR in the sinograms. The amplification of high frequencies by the ramp filter magnifies extraneous noise in projection data. Smoothing roll-off filters, like the Hann filter, are typically applied to ramp-filtered data to control the effects of high frequency noise amplification (20).

Iterative reconstruction has become more common with increased computing power. This method compares successive approximations with the measured sinograms in order to approach the true image incrementally. The first step is to calculate the projections that would have come from a uniform image. These forward projections are compared to the measured sinograms, a difference image is back-projected, and a new approximation is formulated by adding to the uniform image. The forward- and backprojection process is repeated iteratively, and stops when the differences between the measured sinograms and forward projections are within a certain threshold. 
In order to speed up the iterative computation time a method called ordered subsetexpectation maximization (OSEM) was developed. OSEM is a more efficient implementation of the maximum likelihood-expectation maximization (MLEM) algorithm. MLEM uses a likelihood parameter to minimize the difference between a sinogram and forward projections. OSEM speeds up the process by using equally spaced subsets of projections to compare and update the estimation in each iteration instead of the entire set of forward projections.

Multi-slice CT reconstructions are performed using proprietary non-linear interpolation algorithms that balance slice profile, helical pitch, and image noise (21). The simple elegance of FBP cannot be used as multiple slices must be stitched together to form the image.

\subsection{Myocardial Perfusion Imaging}

\subsubsection{Relative Perfusion Imaging}

Conventional MPI with PET is a very useful tool in investigating CAD. This disease is manifested in PET imaging as portions of the myocardium that show reduced tracer activity compared to nearby regions. Unlike dynamic imaging, relative MPI uses a static PET scan where the useful image acquisition begins after the majority of activity is taken up, or trapped in, the myocardial tissue. For example, $a^{82} \mathrm{Rb}$ PET MPI scan is a sum of all the activity from about two minutes into the scan until the end. 
Relative MPI with ${ }^{82} \mathrm{Rb}$ PET has been shown to accurately detect and localize singlevessel CAD (22). However, MPI is known to have reduced sensitivity to detect microvascular disease in patients with diabetes (23) or multi-vessel CAD (24) (25). The extent and severity of disease can be underestimated when MPI is measured relative to the 'most normal' territory. Recent advances in technology and imaging for the tracer used in this study, ${ }^{82} \mathrm{Rb}$, have led to the possibility of routine quantification of myocardial blood flow (MBF) (26), which increases the ability to detect multi-vessel disease (MVD) (27) (28) and provides additional diagnostic information regarding coronary stenosis and ischemia (29).

\subsubsection{Quantitative Blood Flow Imaging}

Quantitative imaging seeks to use known factors, like the behavior of a radiotracer in vivo and the amount of activity at various time-points using dynamic imaging, to evaluate how much blood per unit time brings vital oxygen and nutrients to the heart muscle tissue. Methods of quantifying blood flow in the myocardium with PET have been successful at determining absolute measures of flow (24) (30) (31) and improving detection of disease(25) (26) (27) (32). Rubidium-82 PET imaging has been validated against other myocardial perfusion tracers like nitrogen-13-ammonia $\left({ }^{13} \mathrm{NH}_{3}\right)$ and invasive measurements like microspheres, and has been shown to measure rest and stress MBF accurately (30) (31). 
Flow quantification starts with a dynamic PET scan. The acquired emission data are reconstructed and processed using tracer kinetic analysis. Tracer kinetic analysis, or compartmental modeling, is the process of applying a mathematical model to the bloodheart system dynamics. This enables the measurement of parameters that can ultimately be used to quantify the flow of blood to the LV myocardium. True quantitative analysis depends on an accurate correction to an effect called partial volume (PV) averaging. When accurate image data acquisition, reconstruction, and robust tracer kinetic analysis are applied to dynamic PET scan data, then measurements of $\mathrm{MBF}$ in absolute units of $\mathrm{mL} / \mathrm{min} / \mathrm{g}$ are possible.

\section{Tracer Kinetic Modeling}

Dynamic PET imaging produces measured temporal-spatial distributions of radioactivity called time activity curves (TACs) in a subject at various time points (Fig. 1.5). There is a direct physiologic relationship between the activity levels in various regions of the heart and the biological or biochemical processes. Compartments represent either physical space, chemical forms, or pharmacological states (33). Figure 1.6 shows a generalized two-compartment model that could include all three types of compartments. Examples of a physical space would be the LV cavity or LV myocardium, a chemical form would be fluorodeoxyglucose (FDG, $\mathrm{C}_{6} \mathrm{H}_{11}{ }^{18} \mathrm{FO}_{5}$ ) or FDG-6-phosphate $\left(\mathrm{C}_{6} \mathrm{H}_{12}{ }^{18} \mathrm{FO} \mathrm{O}_{8} \mathrm{P}\right)$, and a 


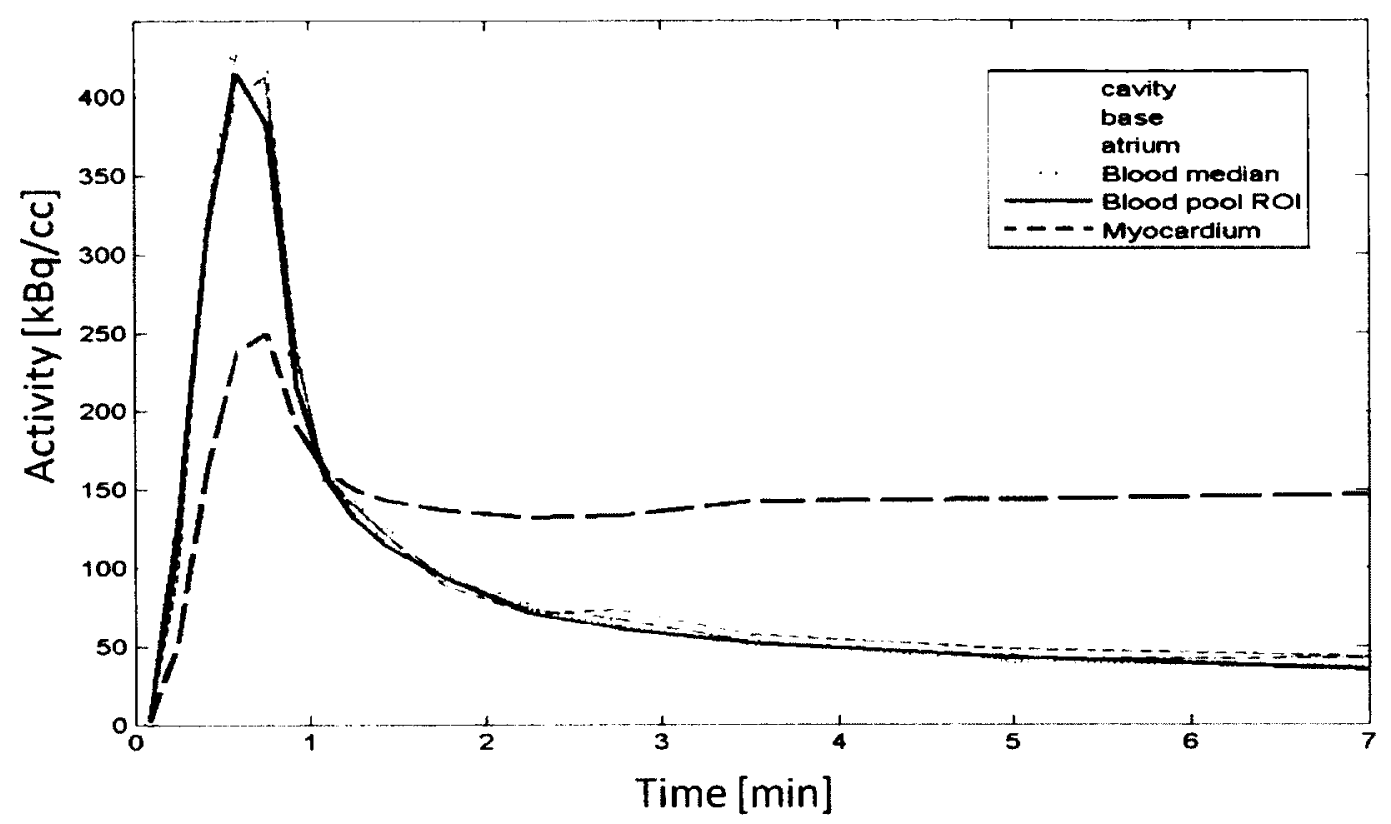

Figure 1.5: A time activity curve showing the amount of activity in absolute units of $\mathrm{kBq} / \mathrm{cm}^{3}$ in various regions in the heart over time.

Rate constant 1

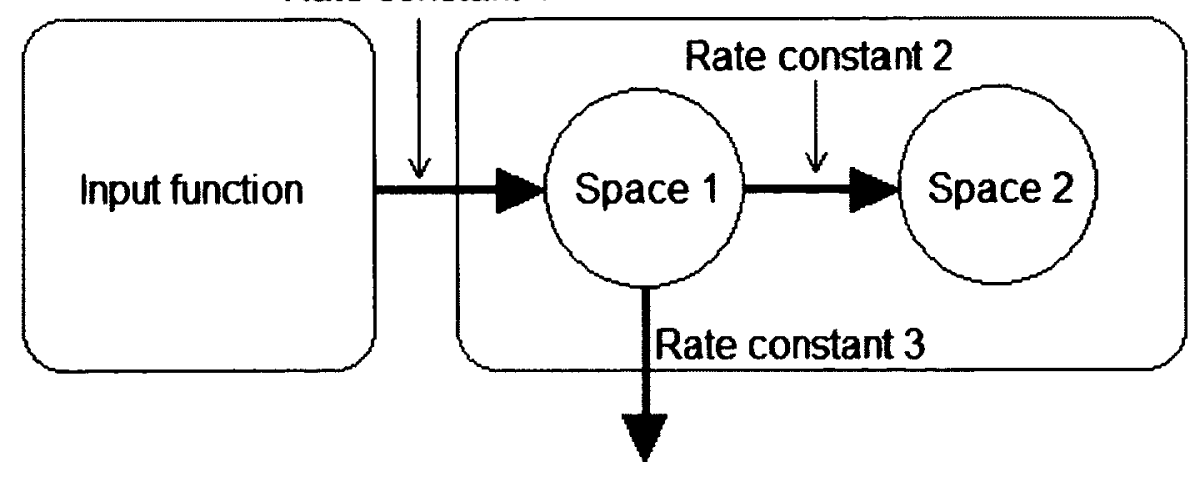

Figure 1.6: A generalized two-compartment tracer kinetic model showing a schematic of the dynamic rate-constant processes between spaces. 
pharmacological state would be bound tracer or unbound tracer in the same physical space. Each compartment is linked by a rate constant $k\left(\min ^{-1}\right)$. The rate constant is a model parameter that describes the speed of tracer exchange or transformation that occurs between compartments. In theory the tracer in the entire compartment is considered to be instantaneously homogeneous, which is impossible. That the compartments achieve homogeneity faster than the rate constant is typically adequate for the models to describe the system. The concentration of tracer in a compartment can be described as a function of time by a first-order differential equation and is governed by conservation of mass. This means that the concentration of tracer is equal to the influx of tracer into, minus the efflux of tracer out of, a compartment in units of inverse time $\left(\mathrm{min}^{-1}\right)$. Blood flow through vessels is described in units of volume per unit time $(\mathrm{mL} / \mathrm{min})$ while MBF, or 'perfusion', is expressed as blood flow per unit mass of tissue $(\mathrm{mL} / \mathrm{min} / \mathrm{g})$.

The uptake and net retention of tracer in tissue depends on tracer extraction and clearance. A simple model relating the parameters for extraction was developed by Renkin and Crone (34) (35). The extraction fraction of a tracer depends on the surface area of a vessel network, $S$, on the permeability of the tracer through the vessels, $P$, and on the flow of blood through the vessels. The simple model predicts that the permeability-surface (PS) product is constant with flow, but experimental results have shown that the PS product increases with flow. An increase in the recruitment of micro- 
vascular capillaries is considered to be the reason for this relationship. The modified extraction fraction is therefore

$$
E(M B F)=1-e^{-(A+B \cdot M B F) / M B F}
$$

where $(A+B \cdot M B F)$ is the flow-dependent PS product, and $A$ and $B$ can be calculated for a tracer by fitting this relationship with experimentally measured uptake data (36).

The mathematics of the generalized model in Figure 1.6 begin with the TAC of a region of interest (ROI) from the dynamic PET scan as an input. In myocardial imaging this represents the tracer concentration of the blood input function. The rates of tracer exchange between the compartments shown in Fig. 1.6 are described by the following differential equations:

$$
\frac{d C_{1}(t)}{d t}=k_{1} C_{\text {input }}(t)-k_{3} C_{1}(t)-k_{2} C_{1}(t)
$$

and

$$
\frac{d C_{2}(t)}{d t}=k_{2} C_{1}(t)
$$

where $d C_{i}(t) / d t$ represents the rate of tracer concentration change in compartment $i$, and $C_{\text {input }}(t), C_{1}(t)$, and $C_{2}(t)$ represent the concentration of tracer in the input function, first compartment, and second compartment respectively. Solutions to the first-order differential equations can be expressed as exponentials convolved with the input function. The values of the rate constants are calculated by fitting the model to the 
measured tissue TAC data using non-linear regression. The final output is the TAC of the tissue of interest, and since the desired parameter is MBF through the heart muscle, the output is the myocardial TAC.

The simplest compartmental model is the model with a single tissue compartment. In comparing a single tissue compartment model to a multiple compartment model, there is a trade-off between bias and precision (33). Fewer compartments might simplify the biological and chemical processes too much, causing the model to yield useless values because of bias. Too many compartments, which model every process exactly, often suffers from high variance in the estimated parameters, making the results unreliable. Fortunately for this reserach, in vivo investigations of a one-compartment model with three of the most common flow tracers, ${ }^{82} \mathrm{Rb}, \mathrm{H}_{2}{ }^{15} \mathrm{O}$, and ${ }^{13} \mathrm{NH}_{3}$, have proven that it is able to model the distribution of these MBF tracers both robustly and accurately (37) (38) (39).

\section{Partial Volume Averaging}

Partial volume (PV) averaging can bias the measured activity concentration if the object, or organ, being imaged is small compared to the reconstructed image resolution. 'Small' is defined as being less than two times the image resolution (16). PET reconstructed image resolution, measured as the point spread function full-width at half-maximum (FWHM), and LV wall thicknesses are both on the order of 6 to 10 millimeters. PV averaging reduces the quantitative accuracy of quantitative MBF due to two factors: 
limited PET resolution and cardiac pumping motion. Cardiac wall motion blurring effects can be mitigated by electrocardiogram (ECG) gated imaging which divides the heart motion into cyclic time bins. This technique, however, is not routinely used in dynamic PET imaging for flow quantification.

Limited PET resolution causes activity from a higher activity region to blur, or 'spill out', into an adjacent region of lower activity. Accordingly, the late-frame activity in the LV myocardium is underestimated. Spillover, in the form of 'spill in', occurs when evaluating a lower activity region, such as the late-frame blood pool, and myocardial wall blurring causes an overestimation of activity. Measurements in the thinnest part of the LV wall at the apex are most affected by PV averaging, as it also has the largest range of wall motion from systole to diastole. Both spill-in and spill-out can cause inaccurate quantification or visual analysis, and may lead to misdiagnosis of disease. Corrections to both of these effects are needed for accurate quantification of MBF.

\section{Myocardial Blood Flow Tracers}

Tracers are chemical compounds or substances that have an affinity for certain biological processes in the body, and are delivered in small amounts that do not alter the function of the process of interest. Localization occurs when molecules of tracer are trapped, as in the case of macro-aggregated albumin when it lodges in the small capillaries of the lung, or is taken up into cells, as in the case of FDG. They come in several forms including naturally occurring compounds, radiolabeled analogs of 
naturally occurring compounds, and radiolabeled drugs. A few of the commonly used MBF tracers for cardiac PET imaging are shown in Table 1.1.

An ideal tracer for blood flow imaging would be freely diffusible across cell membranes and capillary walls and fully extracted from the blood pool. It would also be retained in cells and have a long enough half-life for imaging. Unfortunately, the perfect tracer does not yet exist, and therefore the available ones are used according to their strengths while minimizing the potential effects of their weaknesses (36).

Oxygen-15 water is considered the gold standard for MBF flow quantification with PET in regards to accuracy. The $\mathrm{H}_{2}{ }^{15} \mathrm{O}$ tracer can be produced directly in a cyclotron and separated from by-products (40). It is freely diffusible across capillary and cell membranes and achieves equilibrium between blood pool and tissue quickly. However,

Table 1.1: Characteristics of commonly used PET MBF tracers

\begin{tabular}{lccc}
\hline & ${ }^{13} \mathbf{N}$-ammonia & ${ }^{15} \mathbf{O}-$ water & ${ }^{\mathbf{8 2}} \mathbf{R b}$ \\
\hline Half-life $(\mathrm{min})$ & 9.98 & 2.03 & 1.27 \\
Decay abundance $\left(\boldsymbol{\beta}^{+}\right)$ & $100 \%$ & $100 \%$ & $95.5 \%$ \\
Maximum $\boldsymbol{\beta}^{+}$energy $(\mathrm{MeV})$ & 1.19 & 1.7 & 3.36 \\
Mean $\beta^{+}$energy $(\mathrm{MeV})$ & 0.492 & 0.735 & 1.5 \\
Maximum range in water $(\mathrm{mm})$ & 5.4 & 8.0 & 16.5 \\
Root mean square range $(\mathrm{mm})(\mathbf{4 1})$ & 0.72 & 1.1 & 2.4 \\
Production (42) & On-site cyclotron & On-site cyclotron & Generator \\
\hline Content from (40) unless otherwise noted & & \\
\hline
\end{tabular}


its fast equilibrium state inhibits accurate discernment between blood and tissue for visual myocardial perfusion imaging (MPI) assessments (43). One of its best attributes is that it has an extraction fraction near unity at all physiologic flow rates, which simplifies kinetic modeling and interpretation.

Nitrogen-13 ammonia has also been used extensively for MBF quantification and investigating areas of ischemia. It is also produced in an on-site cyclotron but requires more post-production and radiopharmaceutical synthesis techniques to prepare it for injection. It has a high, though slightly non-linear, decreasing extraction fraction with flow. Nitrogen-13 ammonia diffuses across cell membranes and is trapped in tissue by metabolic processes that convert it to ${ }^{13} \mathrm{~N}$-glutamine. Due to the metabolic processes that take place, a two-tissue compartment model is often used with blood metabolite corrections that are also required to evaluate quantitative MBF.

Rubidium-82 chloride has become the most commonly used cardiac PET perfusion tracer. It is located in the first column of the periodic table and acts as an analog of potassium, which is essential in maintaining cell membrane integrity and heart function. The potassium cation $\left(\mathrm{K}^{+}\right)$is replaced by $\left(\mathrm{Rb}^{+}\right)$in the cellular sodium-potassium pump and transported across cell membranes and retained (44). Clinical use of ${ }^{82} \mathrm{Rb}$ does not require a local cyclotron as it is generator produced. Strontium- $82\left({ }^{82} \mathrm{Sr}\right)$ is loaded onto a tin-oxide or alumina column, immersed in a bath of saline, and decays $\left(t_{1 / 2}=25.5\right.$ days) by electron capture to ${ }^{82} \mathrm{Rb}$. After decay the rubidium is no longer ionically bound to the 
column and dissolves into the surrounding saline, ready for elution and injection into the subject (Fig 1.7) (43). The major cost of using ${ }^{82} \mathrm{Rb}$ as an imaging agent comes from the operation of a high-energy cyclotron, or linear accelerator, for generation of the parent ${ }^{82} \mathrm{Sr}$.

As a blood flow tracer, ${ }^{82} \mathrm{Rb}$ has the highest root mean square range in tissue compared to many others (Table 1.1). Along with a low count density due to the short half-life and imaging time, the high RMS range forces more image smoothing during reconstruction, which results in lower resolution images. The extraction fraction at peak stress is substantially less than ${ }^{13} \mathrm{~N}$-ammonia, which requires a large correction, and may lead to over- or underestimation of MBF (40). Its short half-life (1.27 $\mathrm{min})$ allows for serial imaging within ten-minute intervals. Scanning many patients helps offset the high cost of the generator as it can be eluted about every ten minutes. Imaging studies of the heart under stress conditions are carried out through pharmacological vasodilatation drugs, because bicycle stress is not feasible due to the short tracer half-life.

\subsubsection{Image Processing}

Computer software makes data acquisition, correction, and reconstruction possible. Ideally, post-processing MBF quantification software is highly automated to reduce user influence, but also flexible enough to allow intervention when difficult situations arise with subject data. A number of commercial software packages are available (30) (45) 


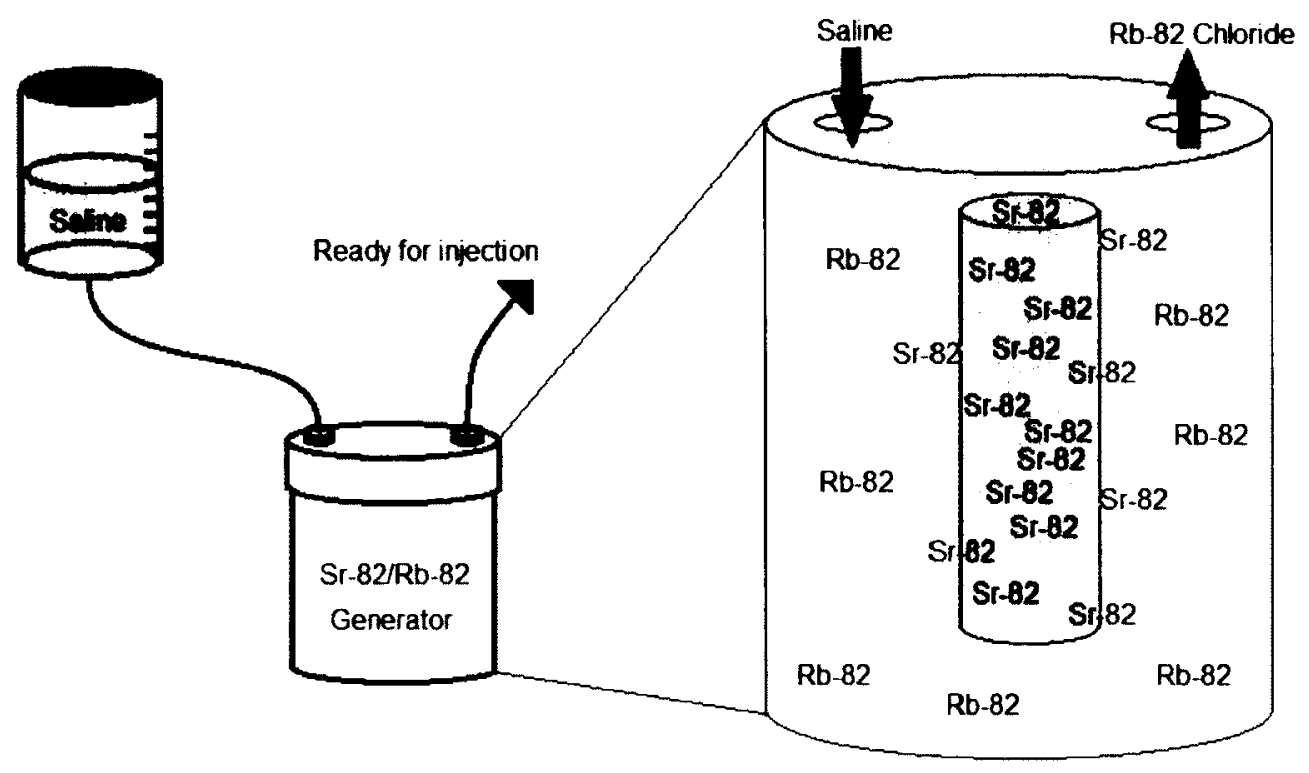

Figure 1.7: A simplified illustration of $a^{82} \mathrm{Sr} /{ }^{82} \mathrm{Rb}$ generator. The $\mathrm{MBF}$ tracer ${ }^{82} \mathrm{RbCl}$ is eluted when saline $(\mathrm{NaCl})$ is flushed through the generator. The chloride anion binds to the rubidium cation to form the solution for injection.

(46) (47) for processing cardiac images, and some investigations have been published comparing their respective merits (48) (49). The UOHI has developed a highly automated image processing package called FlowQuant(C), as described originally by Klein et al (50).

\section{FlowQuant}

The heart does not lie in a convenient orientation for investigation inside the body. The first steps in the cardiac processing workflow are to locate the LV in the dynamic PET scan data, and then to orient it so that the organ can be optimally visualized. An average of the final six to eight minutes of dynamic uptake data are used to visualize the LV 
myocardium. This ensures that the myocardium to blood pool contrast is high, and count statistics are sufficient to reduce image noise. After automatic location of the LV, ellipses are fit to the data in three orthogonal planes: horizontal long axis (HLA), vertical long axis (VLA), and short axis (SA). Figure 1.8 shows the planes and cross-sectional slices. This stage also estimates the length and width of the LV.

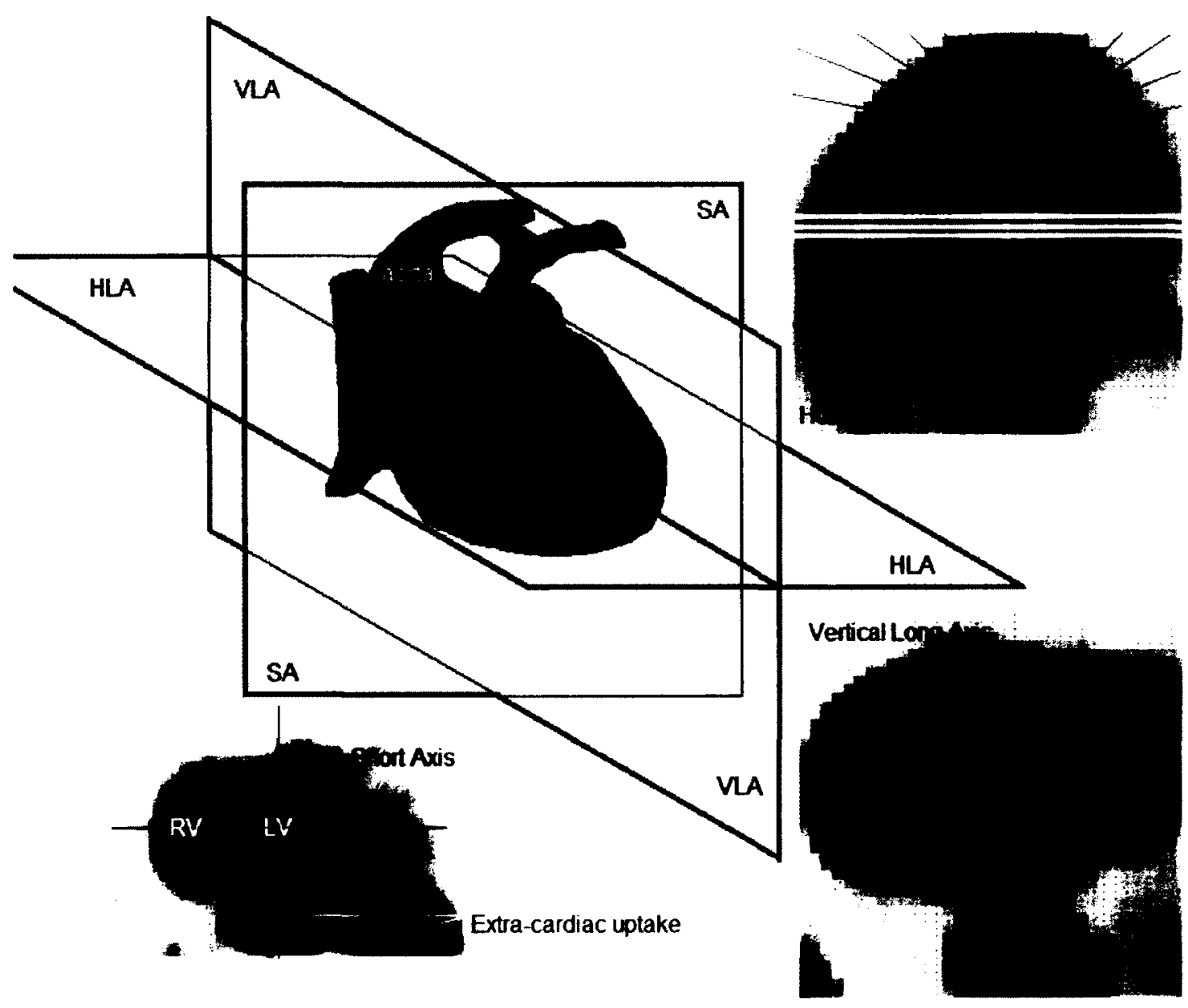

Figure 1.8: Cross-sectional planes of the myocardium and their corresponding PET images. Radial red lines in the Horizontal Long Axis image at top right are conical sampling lines while horizontal white lines are the short axis slices. 
Transverse LV images are reoriented into short axis slices. The apex of the LV, however, is sampled with conical slices to better match the anatomy (Fig. 1.8). The whole LV is segmented, and sampled values are displayed in polar map form (Fig. 1.9) which shows the apex at the center with the outer rings corresponding to basal slices. Myocardial TACS for all time points are typically obtained for 496 polar map sectors. Blood pool regions are centered near the atrium, base, and center, of the LV cavity to sample the blood input function for tracer kinetic modeling. The output of the modeling produces a TAC for the myocardium from which blood flow is extracted (Fig. 1.10). The kinetic modeling steps specific to this research are described in chapter 2.1.

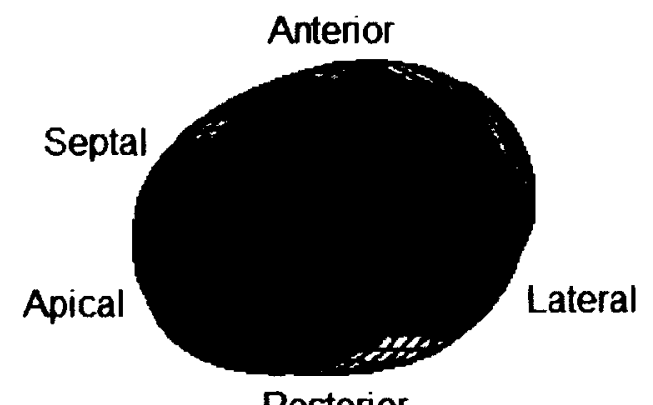

Posterior

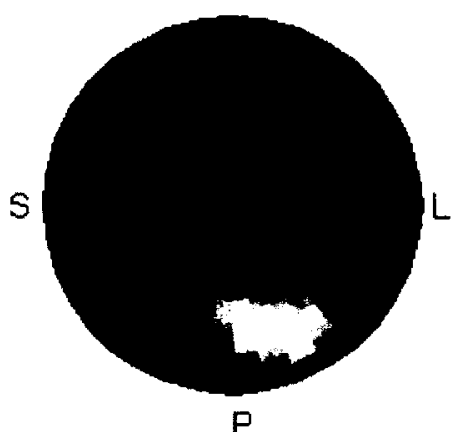

$P$

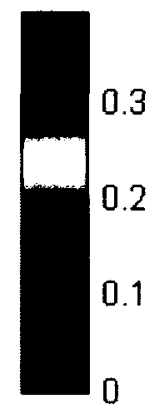

muming

Figure 1.9: Three-dimensional mesh grid of the LV (left) represented in polar map format (right). Color scheme corresponds to flow in $\mathrm{mL} / \mathrm{min} / \mathrm{g}$ as shown with the scale on the right. 


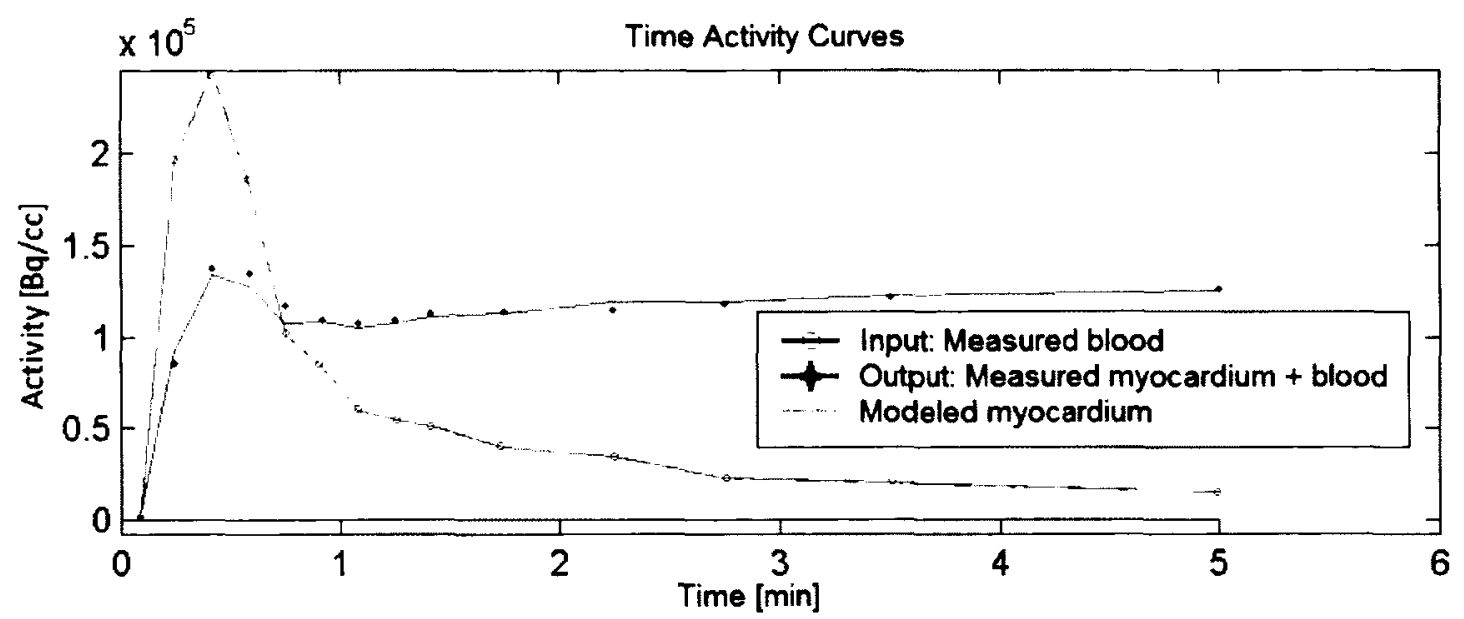

Figure 1.10: Time activity curves for the blood input function (red circles), measured myocardium (blue stars), and modeled myocardium (cyan).

\subsection{Test-Retest Repeatability}

Many factors affect MBF measured with PET. As each person is unique, and the human body is an immensely complex physiologic system, MBF varies between individual people and in the same person at different time-points. The act of making the MBF PET measurement adds variability as well. This intra-subject variation in MBF limits the ability to track the progression of disease and effectiveness of therapies. It also limits the accuracy of setting absolute boundaries between 'normal' and 'diseased' MBF. Test re-test variability, or repeatability, is a very important factor to consider when quantifying MBF for serial or longitudinal imaging. Figure 1.11 shows how similar tracer 

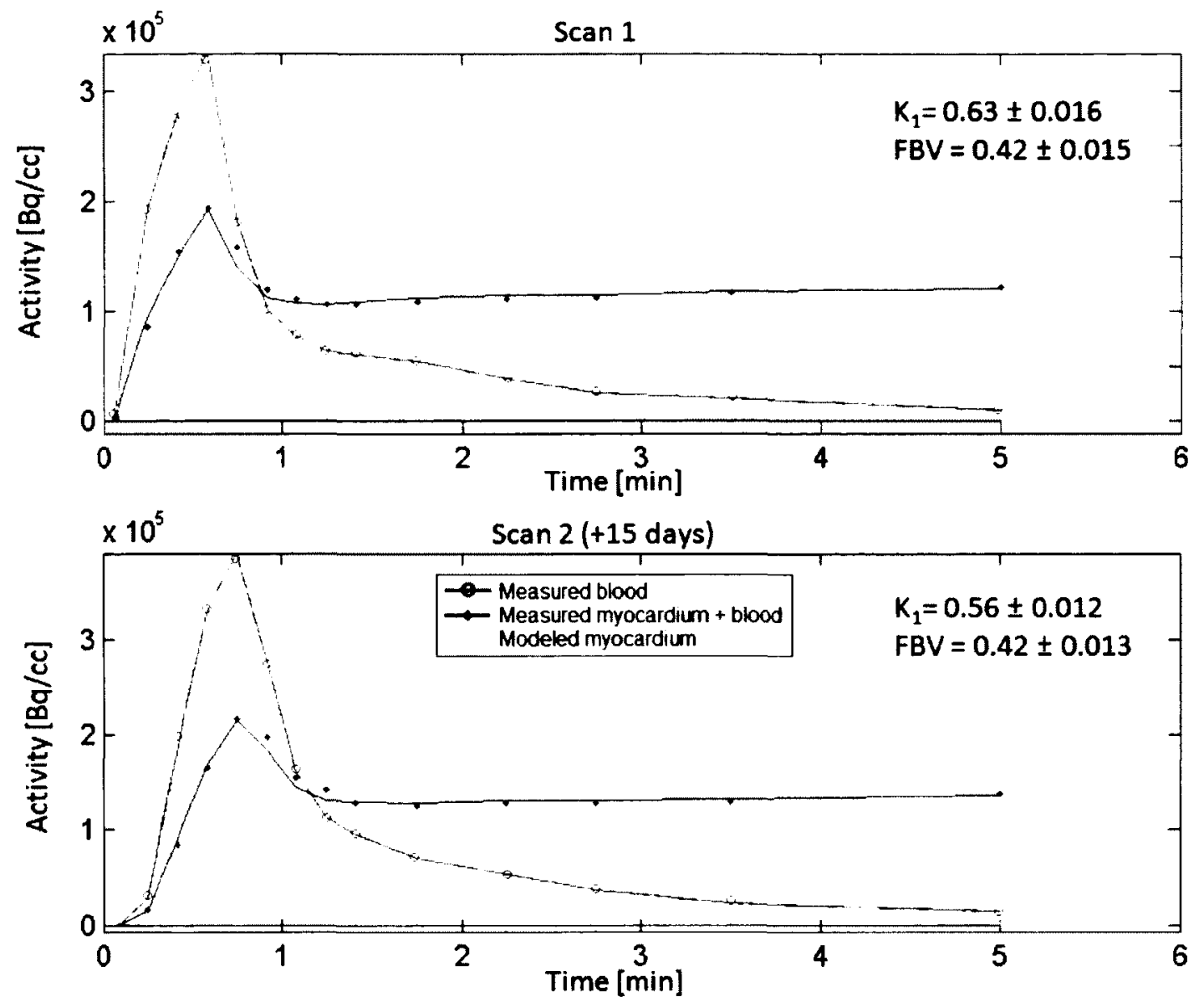

Figure 1.11: Time activity curves of repeated rest scans performed 15 days apart. Similar infusion shapes and blood input parameters (FBV) should result in similar MBF. However, a dissimilar tracer uptake parameter $\left(K_{1}\right)$, which is related to $M B F$, resulted in a $22 \%$ decrease in MBF in scan 2.

infusion TACs in the same subject done fifteen days apart can result in very different MBF results ( $22 \%$ decrease in $\mathrm{MBF}, 0.9$ vs. $1.1 \mathrm{~mL} / \mathrm{min} / \mathrm{g})$. 
Inter- and intra-operator variability for MBF measurement using ${ }^{82} \mathrm{Rb}$ PET was reported using FlowQuant (50). While test-retest data exist for flow quantification in humans using ${ }^{13} \mathrm{NH}_{3}(51)(52)(53)$ and $\mathrm{H}_{2}{ }^{15} \mathrm{O}$ PET (54) (55) (56), there are limited data regarding test-retest repeatability using ${ }^{82} \mathrm{Rb}$ PET in patient studies (30) (57), and no data using the specific system and software developed at the UOHI.

\subsection{Thesis Objectives}

The goals of this research were two-fold. The first was to investigate the same-day testretest repeatability of MBF under resting conditions with ${ }^{82} \mathrm{Rb}$ while optimizing the parameters tested for best results. This will provide evidence to determine the most appropriate protocol for dynamic PET scan reconstruction type, length of data to reconstruct, the effect of hemodynamic adjustment, the utility of a dual bloodmyocardium spillover correction, and where to place the image ROI for the blood input.

The second goal was to investigate the test-retest repeatability effects of heterogeneous myocardial hemodynamic conditions and tracer infusion profiles, with rest and stress MBF imaging performed serially over the course of several weeks or months. Hemodynamic conditions refer to the RPP, while tracer infusion profiles have to do with the timing of injection of tracer into the subject. Very little published literature exists regarding either of these effects (58). These results should provide evidence that uniformity in subject hemodynamic conditions and tracer infusions are key to 
eliminating these sources of variability that affect the quantification and interpretation of MBF measurements with ${ }^{82} \mathrm{Rb}$ PET.

Determining the accuracy of ${ }^{82} \mathrm{Rb}$ PET imaging using a one compartment kinetic model was not a goal of this research. Previous investigation has shown that this technique is accurate (59). However, the effects of bias on MBF measurements, and the effect on test-retest repeatability, due to protocol variations has been examined.

Chapter 2 presents the methods of these two studies and Chapter 3 the results. Chapter 4 is a discussion on the interpretation of the results and the final chapter provides conclusions and proposes future research areas for investigation. 


\section{Chapter 2: Methods}

This chapter describes the methods used to perform two separate studies of test-retest repeatability. The first study, same-day repeatability of resting MBF, investigates the optimal repeatability of PET measured MBF by minimizing the effect of time between scans. The second study determines serial repeatability in MBF at rest and stress and of MFR while investigating the effects of heterogeneous subject hemodynamics and tracer infusion profiles. Since many of the methods were identical in both studies they are presented together, with any differences between studies noted. Some results from the first study were used in forming the methods used for the second study.

\subsection{Patient Cohorts}

Thirty-six subjects were included in the same-day study and thirty-two in the serial study. The studies (same-day, serial) included patients $(n=27,29)$ with suspected CAD and healthy normal volunteers $(n=9,3)$. Each was instructed to refrain from consuming caffeine for twelve hours, as caffeine inhibits the efficacy of the pharmocological vasodilator stress agent, beta-blockers for 24 hours, and food for at least four hours prior to the scan. Each subject gave written informed consent under a cardiac PET registry, or rubidium dosimetry study, approved by the Ottawa Heart Institute Research Ethics Board. 


\subsection{Imaging Protocol}

The serial study subjects underwent the standard clinical procedure of a single rest scan followed by a dipyridamole-induced stress scan at each visit as previously described by Klein et al (59). Same-day study subjects underwent a modified single-session procedure that included three successive scans, two at rest and one under dipyridamole-induced stress. Subjects were purposely not repositioned between scans to limit body motion, changes in heart orientation and resting hemodynamics.

According to the standard clinical procedure, subjects were placed on the scanner couch and positioned with the aid of a CT scout scan. Following the CT scout scan a low-dose (0.4 mSv, effective dose), fast helical ( 1.5 seconds), CT scan was performed at normal end-expiration for attenuation correction of the rest ${ }^{82} \mathrm{Rb}$ scan. Tracer activity (10 $\mathrm{MBq} / \mathrm{kg}$ ) was administered from a ${ }^{82} \mathrm{Sr} / \mathrm{Rb}$ generator (59) intravenously over a 30 second interval using a custom infusion system (60), limiting the coincidence dead-time to less than $50 \%$ to ensure accurate measurement of the first-pass bolus activity. Dynamic rest data (scan 1) were acquired over ten minutes in 3D-mode with fifteen time intervals ( $9 \times 10,3 \times 30,1 \times 60,1 \times 120$, and $1 \times 240$ seconds). Dipyridamole was infused $\left(0.14 \mathrm{mg} / \mathrm{kg} / \mathrm{min}\right.$ for five minutes) to induce hyperemic stress and the same ${ }^{82} \mathrm{Rb}$ PET imaging protocol was repeated. A second low-dose CT scan was performed for attenuation correction of the stress scan data. Fourier rebinning (FORE) was applied to the 3D scan data for more efficient 2D reconstruction. Dynamic images were 
reconstructed using OSEM with four iterations, 24 subsets, and an eight mm Hann filter. One serial study rest-stress study was acquired on the Discovery RX PET-VCT scanner (GE Healthcare, Waukesha, MI) and the remaining 31 on the Discovery PET-CT 690.

The modified, same-day study procedure began with the positioning scout scan and injection of the first resting scan dose of ${ }^{82} \mathrm{Rb}$ tracer activity. The standard protocol, minus the scout scan, commenced immediately at the end of the first rest scan with the first attenuation correction CT scan used for both rest scans. Dynamic images were also reconstructed using FBP, with a twelve $\mathrm{mm}$ Hann filter. Sixteen data sets in this study were acquired on the Discovery RX PET-VCT scanner (GE Healthcare, Waukesha, MI) and the remaining 20 on the Discovery PET-CT 690.

\subsection{Analysis}

Image data were processed using FlowQuant v2.2 (University of Ottawa Heart Institute, ON) as previously reported (50). Dynamic images were reoriented automatically into short-axis views, and the left ventricle (LV) myocardial time-activity-curves (TACS) were measured in 496 polar map sectors, $C_{\text {myo }}(t)$. In the same-day study, blood regions were placed automatically within the left ventricle and atrium cavities to sample the blood input function, $\mathrm{C}_{\mathrm{cav}}(\mathrm{t})$, and $\mathrm{MBF}$ estimated using the tracer kinetic model. In the serial study, only the left atrial cavity was used, based on the optimal protocol determined in the same-day study. The tracer kinetic model used to evaluate absolute blood flow will be described in the next section. 
In the same-day study blood flow and flow reserve results were analyzed in two phases. In the LV blood input phase standard clinical protocol settings (10-minute scan, FBP reconstruction) were used as a baseline for comparison. Protocol changes were investigated using sixteen variations of the following four settings:

i. Six-minute vs. ten-minute scan

ii. FBP vs. OSEM reconstruction

iii. Dual spillover corrected (SOC) vs. uncorrected

iv. RPP adjusted vs. unadjusted.

Rest MBF values were adjusted for the subject-specific RPP as

$$
M B F_{a d j}=M B F \times \frac{\overline{R P P}}{R P P},
$$

where $\overline{R P P}$ is the average RPP across all study subjects (7426 bpm $\cdot \mathrm{mm} \mathrm{Hg}$ ).

The second phase introduced the blood input ROI in the left atrium (LA) (61). The maximum extent of the ROI was a cylindrical shape defined by the average LV diameter. Two frames, taken from the time-frame with maximal activity in the LV cavity and the previous time-frame, were averaged to define the LA blood pool. The useable blood input region was defined as the contiguous pixels with at least $85 \%$ of the maximum pixel activity in the two-frame average ROI (62). Figure 2.1 shows a representative sample image of the standard clinical LV ROI compared to the LA input region. The two most repeatable rest protocols from the LV analysis phase were re-analyzed with the LA input. These four LA and LV protocols, with the corresponding LV SOC on/off pairs, were also used to analyze the single stress scans to evaluate the effects on myocardial flow 
reserve (MFR). In the serial study, all images were analyzed with the previously determined most repeatable protocol: six-minute scan length, SOC, LA input region, with and without the RPP adjustment. Repeatability was assessed with correlation and Bland-Altman coefficients (63).

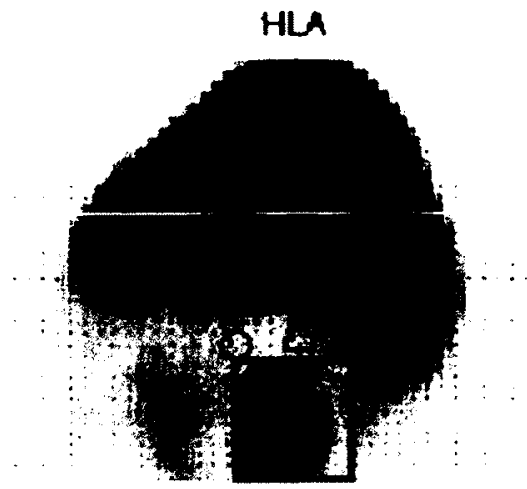

VA

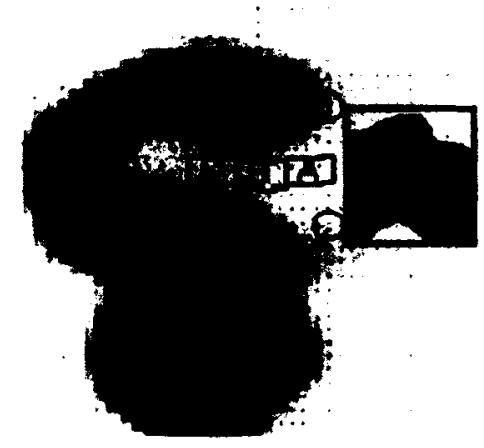

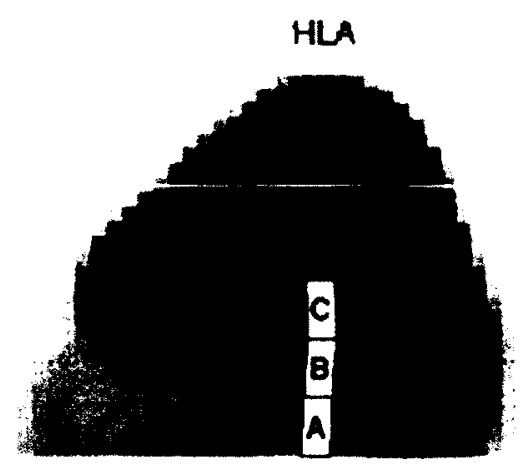

VLA

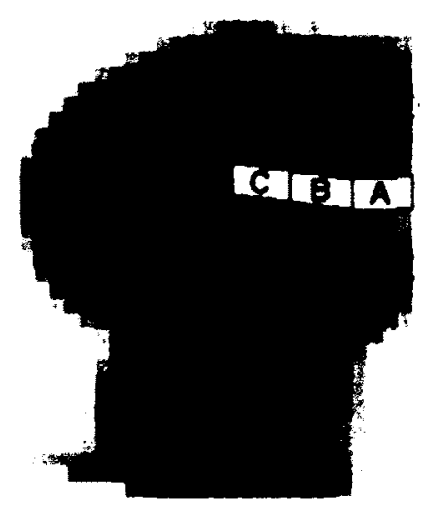

Figure 2.1: Horizontal and vertical long axis sample images showing the maximal extent of the left atrium region of interest (left-blue box) and the $85 \%$ ROI (left-solid red region inside box) for tracer kinetic modeling input function vs. the left ventricle cavity (right-white $\mathrm{CBA}$ ) region of interest (C-cavity, B-base, A-atrium). 


\subsubsection{Kinetic Modeling}

A one-tissue-compartment distribution-volume model of tracer uptake in myocardial tissue was used to describe the ${ }^{82} \mathrm{Rb}$ tracer kinetics (64)

$$
C_{t}(t)=K_{1} e^{-\left(\frac{K_{1}}{D V}\right) t} \otimes C_{c a v}(t)
$$

where $C_{t}(t)$ is the true myocardial tissue activity concentration in $\mathrm{Bq} / \mathrm{g}, K_{1}$ is the ${ }^{82} \mathrm{Rb}$ uptake rate in $\mathrm{mL} / \mathrm{min} / \mathrm{g}, C_{c o v}(\mathrm{t})$ is the concentration of arterial blood measured in the cavity region as a function of time in $\mathrm{Bq} / \mathrm{mL}$, and $\otimes$ is the convolution operator (50). Distribution volume (DV) is the ratio of tissue and blood tracer concentrations after the compartments reach equilibrium. At equilibrium the net exchange of tracer is zero and DV becomes

$$
D V=\frac{K_{1}}{k_{2}}
$$

DV was set to a constant value (specific for each scan) determined by fitting the unconstrained model to the normal uptake region ( $>75 \%$ max) within the polar map.

Uptake, $K_{1}$, and $M B F(\mathrm{~mL} / \mathrm{min} / \mathrm{g})$ are related by an extraction fraction

$$
K_{1}=M B F \times E(M B F),
$$

where

$$
E(M B F)=1-e^{-\left(\frac{P S(M B F)}{M B F}\right)}
$$


The ${ }^{82} \mathrm{Rb}$ extraction function, $E(M B F)$, is a model-specific term that accounts for nonlinear tracer extraction and the tissue permeability surface-area product, $P S(\mathrm{~mL} / \mathrm{min} / \mathrm{g})$, as a function of MBF (34) (35). The PS function was previously determined in human studies (64) (65), as

$$
P S(M B F)=0.63+0.26 \times M B F
$$

making $K_{1}$

$$
K_{1}=\left(1-0.77 e^{-\frac{0.63}{M B F}}\right) M B F
$$

The myocardial image concentration in each polar map sector was modeled as

$$
C_{\text {meas }}(t)=F B V \cdot C_{c a v}(t)+(1-F B V) \cdot C_{t}(t)
$$

where $F B V$ is the estimated fractional blood volume and (1-FBV) was used to correct for regional partial-volume losses in the myocardium (66). The parameters $K_{1}, k_{2}$, and $F B V$ were estimated with equations 3.2 and 3.8 in each sector of the LV myocardium, using weighted non-linear least squares minimization comparing the modeled with the measured myocardium TACs.

\subsubsection{Dual Spillover Correction (SOC)}

Spillover from the myocardial tissue into the LV cavity reduces the quantitative accuracy of the image-derived blood input function. To improve the accuracy, a technique was developed to estimate a pure blood signal for use as input to the kinetic model instead 
of $C_{\text {cav }}(t)(65)$. The proposed method models the myocardium and cavity TACs as mixed partial-volume fractions of pure blood, $C_{b}(t)$ and pure myocardium $C_{t}(t)$ signals (65). The measured myocardium and blood pool regions are modeled as

$$
C_{\text {meas }}(t)=F B V \cdot C_{b}(t)+(1-F B V) \cdot C_{t}(t),
$$

and

$$
C_{c a v}(t)=\beta \cdot C_{b}(t)+(1-\beta) \cdot C_{t}(t),
$$

where $C_{\text {cov }}(t)$ is the mixed blood signal in the cavity and $\beta$ is the partial-volume fraction of pure blood signal in the cavity region of interest. Equations 3.9 and 3.10 were combined to generate the pure blood signal, $C_{b}(t)$, from the measured functions, $C_{\text {meas }}(t)$ and $C_{\text {cav }}(t)$,

$$
C_{b}(t)=\frac{(1-F B V) \cdot C_{c a v}(t)-(1-\beta) \cdot C_{m e a s}(t)}{\beta \cdot(1-F B V)-(1-\beta) \cdot F B V} .
$$

First, the parameters $K_{1}, k_{2}, F B V$, and $\beta$ were estimated using the average LV polar-map data $C_{\text {meas }}(t)$, using weighted non-linear least squares minimization with equations 3.2 , $3.9,3.11$. Then in a second step, using the $\beta$ value estimated above, the corresponding pure blood signal $C_{b}(t)$ from equation 3.11 was substituted in place of $C_{c a v}(t)$ to estimate regional MBF in each polar map sector with equations 3.2 and 3.8 . 


\subsubsection{Gamma Variate Function}

The shape of the tracer infusion profile was used in the serial study as a comparison parameter. Changes in the shape of the TAC data from scan 1 to scan 2 due to abnormal

${ }^{82} \mathrm{Rb}$ infusions could affect the ability of the model to accurately describe the system. This investigation aimed to assess repeatability under similar and dissimilar infusion conditions.

A modified gamma variate (GV) function was fit to the blood time activity data points to characterize the TAC infusion profile. The shape of this function models the rise of activity and the sharp peak as a first-pass bolus, or timed injection, clears the IV cavity (67). It has the form

$$
y\left(t^{\prime}\right)=y_{\max } t^{\prime \alpha} e^{\alpha\left(1-t^{\prime}\right)}
$$

where $y_{\max }$ is the peak activity, $\alpha$ is a free parameter, and $t^{\prime}$ is defined as

$$
t^{\prime}=\frac{t-t_{0}}{t_{\max }-t_{0}}
$$

The parameters $t_{0}$ and $t_{\max }$ are the activity appearance times and peak activity time, respectively. The rise-time, from activity appearance to peak activity, was calculated as $t_{\text {max }}-t_{0}$. A reduced chi-squared goodness-of-fit statistic $\left(\chi_{v}^{2}\right)$ was calculated between the blood data points and the modified GV function, from $t_{0}$ to the last point before the onset of tracer recirculation. A second infusion profile parameter, tracer clearance-time 
$\left(T_{c}\right)$, was calculated as the time from $t_{0}$ up to an endpoint corresponding to $50 \%$ of the peak blood activity.

A variable transformation from the modified GV function to the original GV function (68) was implemented to extract a third tracer infusion profile parameter, mean transit-time (MTT), from the fit. The original GV function has the form

$$
y(t)=C\left(t-t_{0}\right)^{\alpha} e^{-\frac{\left(t-t_{0}\right)}{\beta}}
$$

where $C$ is a constant scale factor and $\alpha$ and $\beta$ are parameters determined during model fitting. The MTT is the average time for a molecule of tracer to travel to the ROI and was calculated from

$$
M T T=t_{0}+\beta(\alpha+1)
$$

Figure 2.2 shows the parameters that were extracted from the GV, and modified GV, function fits.

\subsubsection{Hemodynamics}

Differences in hemodynamic conditions from scan 1 to scan 2 may represent an additional factor affecting repeatability of MBF at rest and stress (52). Therefore, differences in RPP between repeated rest and stress scans were calculated and the resulting effects on MBF were investigated. 


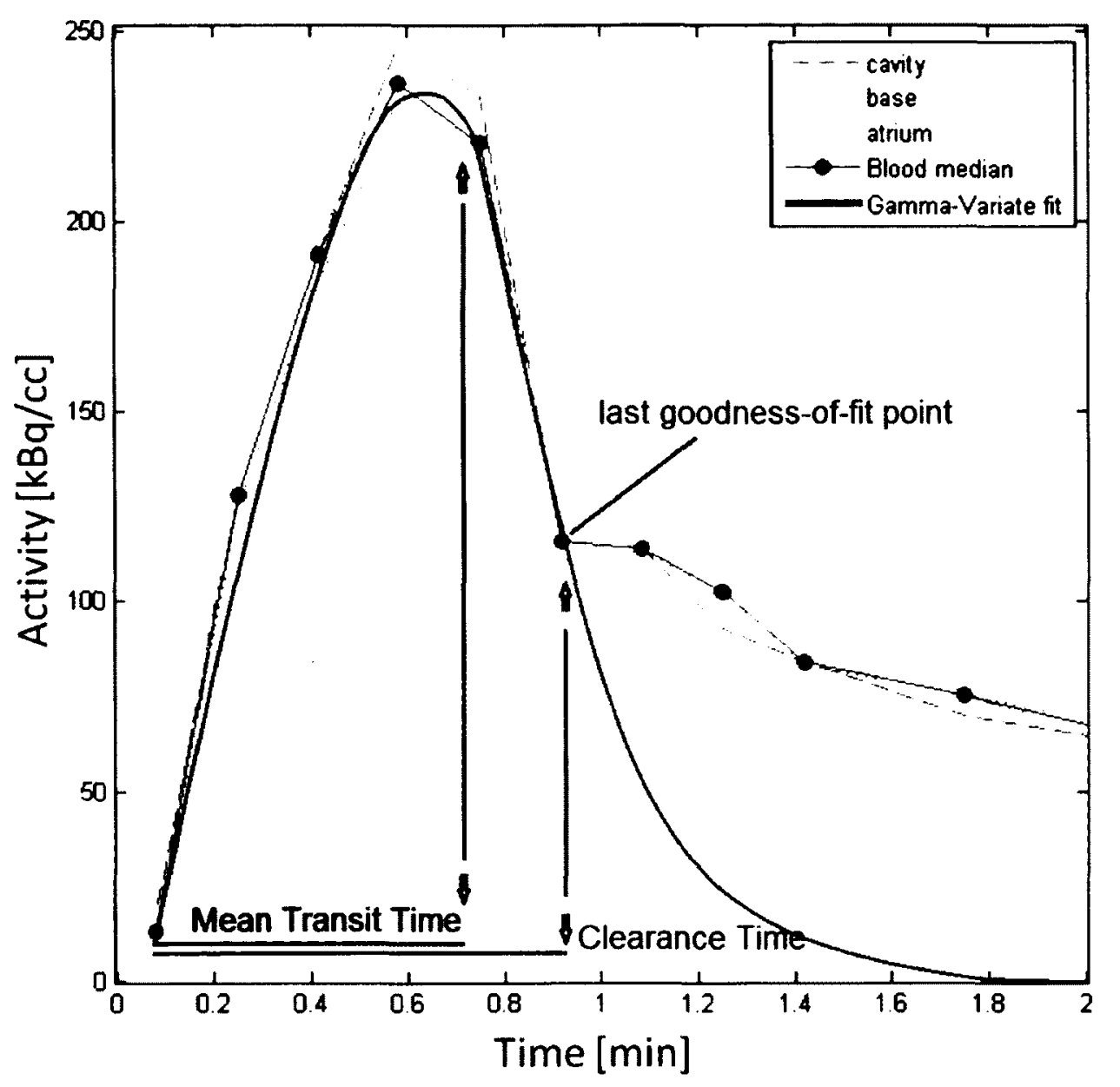

Figure 2.2: A representative scan showing the tracer infusion profile parameters investigated. The tracer clearance-time measures the time it takes for the activity concentration to return to half the height of the peak. The mean transit-time is the average time for the tracer to travel to the region of interest. The goodness-of-fit measures the squared sum of errors between the Blood Median and Gamma-Variate fit from time zero to the last point before tracer recirculation begins. 


\subsubsection{Statistical Analysis}

Paired and un-paired Student's t-test and multiple factor ANOVA (with interactions) were used to analyze the segmental MBF data where appropriate. The F-test was used to compare the test-retest variance between scans. Bonferroni correction was applied to account for multiple statistical tests. Correlation plots (Pearson's $r^{2}$ ) were used to show agreement between datasets. Bland-Altman plots were used to compare testretest differences, and to provide a visual representation of the repeatability coefficient $(\mathrm{RPC}=1.96 \times$ standard deviation of differences; discussed in Appendix $A)$ and the coefficient of variation (CV, standard deviation / mean $\times 100 \%$ ) (63). Differences were considered statistically significant when $\mathrm{p}<0.05$. 


\section{Chapter 3: Results}

This chapter describes the results of two separate studies of test-retest repeatability. The first study, same-day repeatability of resting MBF, investigated the optimal repeatability of PET measured MBF by eliminating the effect of time between scans. The second study determined the repeatability of serial MBF measurements at rest and stress, and of MFR while investigating the effects of heterogeneous subject hemodynamics and tracer infusion profiles. The results of the same-day study have been accepted for publication in the Journal of Nuclear Cardiology (1).

\subsection{Demographics}

The subject demographics and hemodynamics are summarized in Tables 3.1 to 3.4. The age range of same-day study subjects was $27-76$ years: 45 - 76 for CAD patients and 27 - 60 for normal volunteers. The difference between male and female RPP was significant, both in scan 1 and scan $2(p=0.03)$. The difference in RPP between CAD patients and normal volunteers was not significant for either scan 1 or $\operatorname{scan} 2(p=0.1)$. Scan 1 to scan 2 RPP differences were different $(p=0.05)$.

The age range of serial study subjects was $34-80$ years with a range of $42-50$ years for normal volunteers. Time between scans ranged from fifteen to 541 days (mean $194 \pm$ 147 days). Rate-pressure-product differences between male and female subjects, and CAD patients and normal volunteers, were not significant for either scan 1 or scan 2, 
rest or stress ( $p>0.06)$. Scan 1 to scan 2 RPP differences at rest and stress were also not statistically significant $(p>0.5)$.

Table 3.1: Same-day study subject demographics

\begin{tabular}{|c|c|c|}
\hline Demographics & Age (v) & BMI \\
\hline males $(n=24)$ & $55 \pm 13$ & $30.3 \pm 5.3$ \\
\hline females $(n=12)$ & $57 \pm 12$ & $33.7 \pm 8.7$ \\
\hline normal volunteers $\left(m=6^{a}, f=3\right)$ & $41 \pm 9^{*}$ & $26.6 \pm 3.8^{*}$ \\
\hline CAD patients $(m=18, f=9)$ & $60 \pm 9$ & $33.0 \pm 6.7$ \\
\hline \multicolumn{3}{|c|}{$\begin{array}{l}\text { Values are mean } \pm \text { standard deviation, } \mathrm{BMI}=\text { Body Mass } \mathrm{Index}\left(\mathrm{kg} / \mathrm{m}^{2}\right) \\
{ }^{2} \text { Two normal volunteers had high total cholesterol }(\mathrm{TCH}>5.2 \mathrm{~mol} / \mathrm{L}) \text { and high } \\
\text { triglycerides }(\mathrm{TGC}>1.70 \mathrm{mmol} / \mathrm{L}) \\
{ }^{*} p<0.05 \text { normal volunteers vs. CAD patients }\end{array}$} \\
\hline
\end{tabular}

Table 3.2: Serial study subject demographics

\begin{tabular}{llc}
\hline Demographics & Age $(y)$ & BMI \\
\hline males $(n=22)$ & $59 \pm 12$ & $29.0 \pm 4.8$ \\
females $(n=10)$ & $63 \pm 12$ & $26.9 \pm 5.7$ \\
normal volunteers $(m=1, f=2)$ & $47 \pm 5^{*}$ & $24.2 \pm 7.4$ \\
CAD patients $(m=21, f=8)$ & $61 \pm 12$ & $28.8 \pm 4.8$ \\
\hline Values are mean \pm standard deviation, BMI = Body Mass $\left(\mathrm{ndex}\left(\mathrm{kg} / \mathrm{m}^{2}\right)\right.$ & \\
& & \\
\end{tabular}


Table 3.3: Same-day study subject hemodynamics

\begin{tabular}{lcc}
\hline & \multicolumn{2}{c}{ Rest } \\
\hline Hemodynamics & Scan 1 & Scan 2 \\
\hline Heart rate $(\mathrm{bpm})$ & $64 \pm 14$ & $65 \pm 13$ \\
Systolic blood pressure $(\mathrm{mm} \mathrm{Hg})$ & $114 \pm 17$ & $116 \pm 17$ \\
RPP (bpm $\times \mathrm{mm} \mathrm{Hg})$ & $7327 \pm 2293$ & $7525 \pm 2368$ \\
RPP males & $6738 \pm 2202^{*}$ & $6922 \pm 2311^{*}$ \\
RPP females & $8505 \pm 2077$ & $8729 \pm 2074$ \\
RPP normal volunteers & $6226 \pm 1417$ & $6387 \pm 1407$ \\
RPP CAD patients & $7693 \pm 2428$ & $7904 \pm 2518$ \\
\hline
\end{tabular}

Values are mean \pm standard deviation, $\mathrm{bpm}=$ heart-beats per minute, $\mathrm{mm} \mathrm{Hg}=$ millimeters of mercury, $\mathrm{RPP}=$ rate-pressure-product

$* p=0.03$ males vs. females

Table 3.4: Serial study subject hemodynamics

\begin{tabular}{lcccc}
\hline & \multicolumn{2}{c}{ Rest } & \multicolumn{2}{c}{ Stress } \\
\hline Hemodynamics & Scan 1 & Scan 2 & Scan 1 & Scan 2 \\
\hline Heart rate $(\mathrm{bpm})$ & $61 \pm 10$ & $62 \pm 11$ & $79 \pm 12$ & $79 \pm 11$ \\
SBP $(\mathrm{mm} \mathrm{Hg})$ & $114 \pm 15$ & $114 \pm 19$ & $118 \pm 19$ & $117 \pm 20$ \\
RPP $(\mathrm{bpm} \times \mathrm{mmHg})$ & $6916 \pm 1281$ & $7089 \pm 1932$ & $9244 \pm 1874$ & $9120 \pm 1764$ \\
RPP males & $6627 \pm 1327$ & $6999 \pm 2031$ & $9153 \pm 2071$ & $9165 \pm 2005$ \\
RPP females & $7553 \pm 943$ & $7286 \pm 1781$ & $9445 \pm 1422$ & $9021 \pm 1148$ \\
RPP normal volunteers & $6952 \pm 1636$ & $6387 \pm 647$ & $9203 \pm 713$ & $9236 \pm 483$ \\
RPP CAD patients & $6913 \pm 1275$ & $7161 \pm 2012$ & $9248 \pm 1963$ & $9108 \pm 1851$ \\
\hline Values are mean \pm standard deviation, bpm = heart-beats per minute \\
SBP = systolic blood pressure, mm Hg = millimeters of mercury \\
RPP = rate-pressure-product
\end{tabular}




\subsection{Protocol Effects on MBF and MFR}

\subsubsection{Same-Day Study}

Table 3.5 summarizes the same-day scan 1 and scan 2 resting MBF results for the imaging protocols evaluated with the LV cavity input function. Multi-factor ANOVA showed differences in male vs. female $(p<0.01)$ and normal vs. CAD patients $(p<0.05)$ MBF values, as well as a systematic decrease in MBF using the SOC $(p<0.01)$. Other parameters (reconstruction method, scan length, scan number, RPP adjustment) did not have a significant effect on the mean MBF values ( $p>0.18$ ). There were significant interactions of gender and disease status with RPP $(p<0.01)$.

The population standard deviation values (SD) shown in Table 3.5 were not significantly different between test-retest scans, reconstruction methods, scan length, or between population subgroups, i.e., gender and disease status. The mean SDs for all methods for scan 1 and 2 where 0.26 and 0.33 respectively, while RPP adjusted SDs for scan 1 and 2 were 0.17 and 0.21 respectively. The mean MBF was essentially unchanged after RPP adjustment, but the population sample SD values decreased significantly from 0.26 to $0.17 \mathrm{~mL} / \mathrm{min} / \mathrm{g}$ for scan 1 , and from 0.33 to $0.21 \mathrm{~mL} / \mathrm{min} / \mathrm{g}$ for scan 2 ( $p<0.01$ ). Therefore, individual RPP values accounted for a significant fraction $(\sim 1 / 3)$ of the population variability in resting MBF. 
Figure 3.1 shows a box-plot of resting scan $1 \mathrm{MBF}$ by gender with and without RPP adjustment, using six-minute scans with OSEM reconstruction and dual-spillover correction (OSEM-6-SOC). The mean MBF was 50\% higher in females vs. males in scan $1(p<0.001)$ and scan $2(p=0.005)$. After the RPP adjustment was applied, however, neither the difference in scan $1(p=0.07)$ nor scan $2(p=0.28)$ remained statistically significant.

Table 3.5: Same-day rest MBF values with and without RPP-adjustment

\begin{tabular}{|c|c|c|c|c|c|c|}
\hline $\begin{array}{c}\text { Reconstruction } \\
\text { Method }\end{array}$ & $\begin{array}{c}\text { Scan Length } \\
\text { (min) }\end{array}$ & $\begin{array}{c}\text { SOC } \\
*\end{array}$ & $\begin{array}{c}\text { MBF } \\
\text { Scan } 1\end{array}$ & $\begin{array}{l}\text { MBF RPP } \\
\text { Scan 1** }\end{array}$ & $\begin{array}{c}\text { MBF } \\
\text { Scan } 2\end{array}$ & $\begin{array}{l}\text { MBF RPP } \\
\text { Scan 2*** }\end{array}$ \\
\hline FBP & 10 & SOC & $0.74 \pm 0.27$ & $0.75 \pm 0.18$ & $0.76 \pm 0.37$ & $0.75 \pm 0.21$ \\
\hline FBP & 10 & & $0.82 \pm 0.29$ & $0.83 \pm 0.16$ & $0.83 \pm 0.36$ & $0.83 \pm 0.21$ \\
\hline FBP & 6 & SOC & $0.74 \pm 0.27$ & $0.75 \pm 0.19$ & $0.76 \pm 0.33$ & $0.76 \pm 0.21$ \\
\hline FBP & 6 & & $0.87 \pm 0.28$ & $0.88 \pm 0.17$ & $0.86 \pm 0.32$ & $0.87 \pm 0.20$ \\
\hline OSEM & 10 & SOC & $0.78 \pm 0.25$ & $0.79 \pm 0.19$ & $0.78 \pm 0.33$ & $0.79 \pm 0.23$ \\
\hline OSEM & 10 & & $0.81 \pm 0.26$ & $0.82 \pm 0.15$ & $0.81 \pm 0.36$ & $0.82 \pm 0.23$ \\
\hline OSEM & 6 & SOC & $0.76 \pm 0.25$ & $0.77 \pm 0.18$ & $0.75 \pm 0.28$ & $0.76 \pm 0.21$ \\
\hline OSEM & 6 & & $0.86 \pm 0.26$ & $0.88 \pm 0.18$ & $0.86 \pm 0.33$ & $0.87 \pm 0.22$ \\
\hline \multicolumn{7}{|c|}{$\begin{array}{l}\text { SOC }=\text { dual spillover corrected, MBF values are } \mathrm{mL} / \mathrm{min} / \mathrm{g} \text {, va } \\
\text { deviation, RPP }=\text { rate-pressure-product, MBF RPP are adjusted as: } \\
{ }^{*} p<0.01 \text { for all SOC vs. no SOC protocol pairs } \\
{ }^{* *} p=0.01 \text { sample variance decreased vs. MBF for all scan } 1 \text { pairs } \\
{ }^{* * *} p<0.01 \text { sample variance decreased vs. MBF for all scan } 2 \text { pairs }\end{array}$} \\
\hline
\end{tabular}




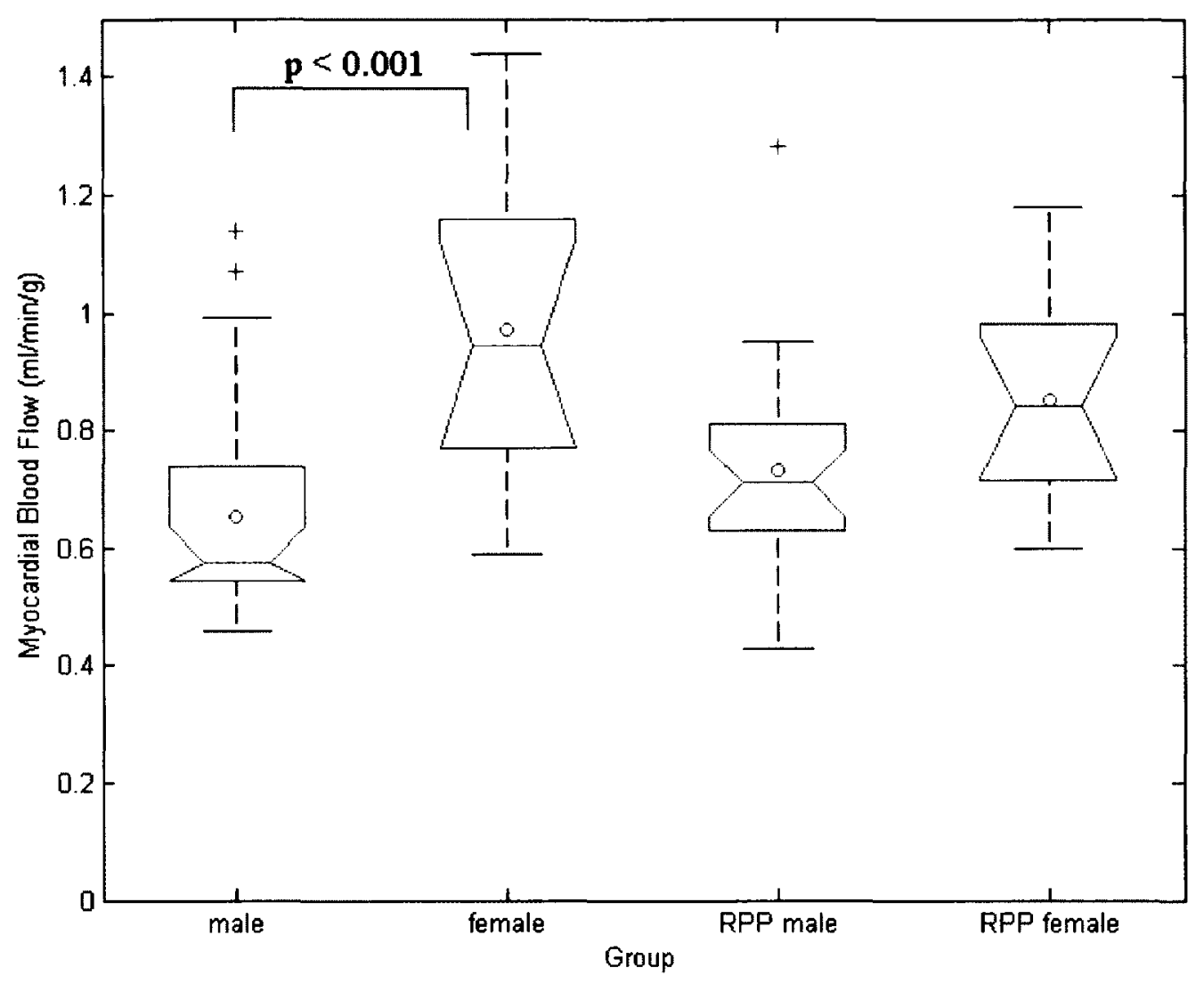

Figure 3.1: Notched box-plot comparison of same-day scan 1 MBF values between male and female subjects, with and without RPP adjustment using six-minute scan-time, OSEM reconstruction and dualspillover correction. Outliers (+ symbols) are flow values $>1.5 \times$ interquartile range. Resting mean MBF values (circles) are significantly higher in females vs. males before adjustment for RPP (0.97 vs. 0.65 $\mathrm{mL} / \mathrm{min} / \mathrm{g} ; \mathrm{p}<0.001$ ) but not after $(0.85 \mathrm{vs} .0 .73 \mathrm{~mL} / \mathrm{min} / \mathrm{g} ; \mathrm{p}=0.07$ ). See Appendix B for a box-plot tutorial. 
While the present study was unable to incorporate a second stress scan with the standard administration of aminophylline (which reverses the effects of dipyridamole), the single stress scan was paired with both rest scans to simulate single-session repeated MFR measurements. Four subjects did not complete stress scans reducing the population to thirty-two for stress and MFR. Table 3.6 shows the MFR results for the two optimal LV, and corresponding LA input protocols.

Multi-factor ANOVA showed significant differences in stress MBF values for male vs. female $(p<0.001)$, normal vs. CAD patients $(p<0.001)$, and significant interactions of gender with disease status $(p<0.04)$. Other parameters (reconstruction method, scan length, scan number) did not have a significant effect on the mean MBF values ( $p>$ $0.26)$. There were significant differences in MFR values for OSEM vs. FBP $(p=0.001)$,

Table 3.6: Same-day study stress and myocardial flow reserve (MFR)†

\begin{tabular}{ccccccccc}
\hline $\begin{array}{c}\text { Recon } \\
\text { Method* }\end{array}$ & $\begin{array}{c}\text { Scan } \\
\text { Time }\end{array}$ & $\begin{array}{c}\text { Input } \\
* *\end{array}$ & SOC & Stress & $\begin{array}{c}\text { MFR } \\
\text { Scan 1 }\end{array}$ & $\begin{array}{c}\text { MFR RPP } \\
\text { Scan 1 }\end{array}$ & $\begin{array}{c}\text { MFR } \\
\text { Scan 2 }\end{array}$ & $\begin{array}{c}\text { MFR RPP } \\
\text { Scan 2 }\end{array}$ \\
\hline FBP & 10 & LV & & $2.17 \pm 1.16$ & $2.62 \pm 1.04$ & $2.60 \pm 1.09$ & $2.60 \pm 1.00$ & $2.58 \pm 1.03$ \\
FBP & 10 & LV & SOC & $2.01 \pm 0.90$ & $2.77 \pm 1.02$ & $2.71 \pm 0.94$ & $2.77 \pm 1.05$ & $2.70 \pm 0.95$ \\
FBP & 10 & LA & & $2.19 \pm 1.22$ & $2.64 \pm 1.04$ & $2.93 \pm 2.14$ & $2.64 \pm 1.03$ & $2.93 \pm 2.02$ \\
OSEM & 6 & LV & SOC & $1.91 \pm 0.85$ & $2.58 \pm 0.98$ & $2.53 \pm 0.91$ & $2.63 \pm 1.03$ & $2.58 \pm 0.99$ \\
OSEM & 6 & LV & & $2.04 \pm 0.96$ & $2.37 \pm 0.92$ & $2.37 \pm 1.03$ & $2.43 \pm 1.03$ & $2.42 \pm 1.08$ \\
OSEM & 6 & LA & SOC & $1.94 \pm 1.12$ & $2.38 \pm 0.96$ & $2.32 \pm 0.83$ & $2.33 \pm 0.88$ & $2.27 \pm 0.75$ \\
\hline
\end{tabular}

+ A single stress scan paired with repeated rest scans. Scan Time is in minutes, $S O C=$ spillover correction, Myocardial flow reserve (MFR) is unit-less ratio of stress/rest MBF, MFR RPP calculated using rate-pressure-product adjusted MBF ${ }^{*} p=0.001$ effect by ANOVA in MFR for FBP vs. OSEM ${ }^{* *} p<0.005$ effect by ANOVA in MFR for LV vs. LA 
normal vs. CAD patients ( $p<0.001)$, male vs. female $(p<0.001)$, LV vs. LA input $(p=0.004)$, as well as significant interactions between reconstruction method, disease status, gender, and blood input $(p<0.02)$.

\subsubsection{Serial Study}

The scans from subject number 30 were removed from the study because the acquisition of data commenced near the peak LV blood pool activity. The GV function could not be fit to the data to extract tracer infusion profile parameters and it is known that the kinetic modeling is not accurate without the early blood input data. The population $(n=31)$ mean MBF values for rest and RPP adjusted rest were $0.69 \pm 0.26$ $\mathrm{mL} / \mathrm{min} / \mathrm{g}$ and $0.69 \pm 0.21 \mathrm{~mL} / \mathrm{min} / \mathrm{g}$ respectively for scan 1 and $0.66 \pm 0.21 \mathrm{~mL} / \mathrm{min} / \mathrm{g}$ and $0.68 \pm 0.22 \mathrm{~mL} / \mathrm{min} / \mathrm{g}$ respectively for scan $2(p=N S)$. Stress flow was significantly increased over rest flow at $1.72 \pm 0.84 \mathrm{~mL} / \mathrm{min} / \mathrm{g}$ for scan 1 and $1.64 \pm 0.73 \mathrm{~mL} / \mathrm{min} / \mathrm{g}$ for scan 2 ( $p<0.001)$. Myocardial flow reserve and RPP adjusted MFR for scan 1 and scan 2 were $2.52 \pm 0.83$ and $2.45 \pm 0.74$ and $2.54 \pm 0.95$ and $2.46 \pm 0.88$ respectively $(p=$ NS).

\section{Infusion Profiles}

The mean rise-time of activity ( $34 \pm 6$ for rest and $32 \pm 6$ seconds for stress scans), from scan start to peak activity, agreed well with the 30-second tracer infusion. Rise-time values less than 30 seconds were typically due to late starts in data acquisition. Average 
values for the mean transit-time were $42 \pm 12 \mathrm{~s}$ for rest and $45 \pm 19 \mathrm{~s}$ for stress ( $p=N S$ ). The mean clearance-times were $53 \pm 14 \mathrm{~s}$ for rest and $54 \pm 11 \mathrm{~s}$ for stress $(p=N S)$.

Tracer infusion profile curves were characterized by extracting three parameters from the GV function fit to the blood time activity data. Figure 3.2 shows box plots of the exclusion analyses with three exclusion levels: $2.25 \times$ the interquartile range (IQR), 1.5 IQR, and 0.75 IQR. For normally distributed data, 2.25 IQR, 1.5 IQR, and 0.75 IQR are approximately three, two, and one standard deviations, respectively.

Profiles from scan 1 and scan 2 were considered heterogeneous and excluded if the difference between the parameters exceeded the whisker limits from the nearest quartile. Five subjects were excluded at $2.25 \mathrm{IQR}$, eight at $1.5 \mathrm{IQR}$, and sixteen at 0.75 IQR. 


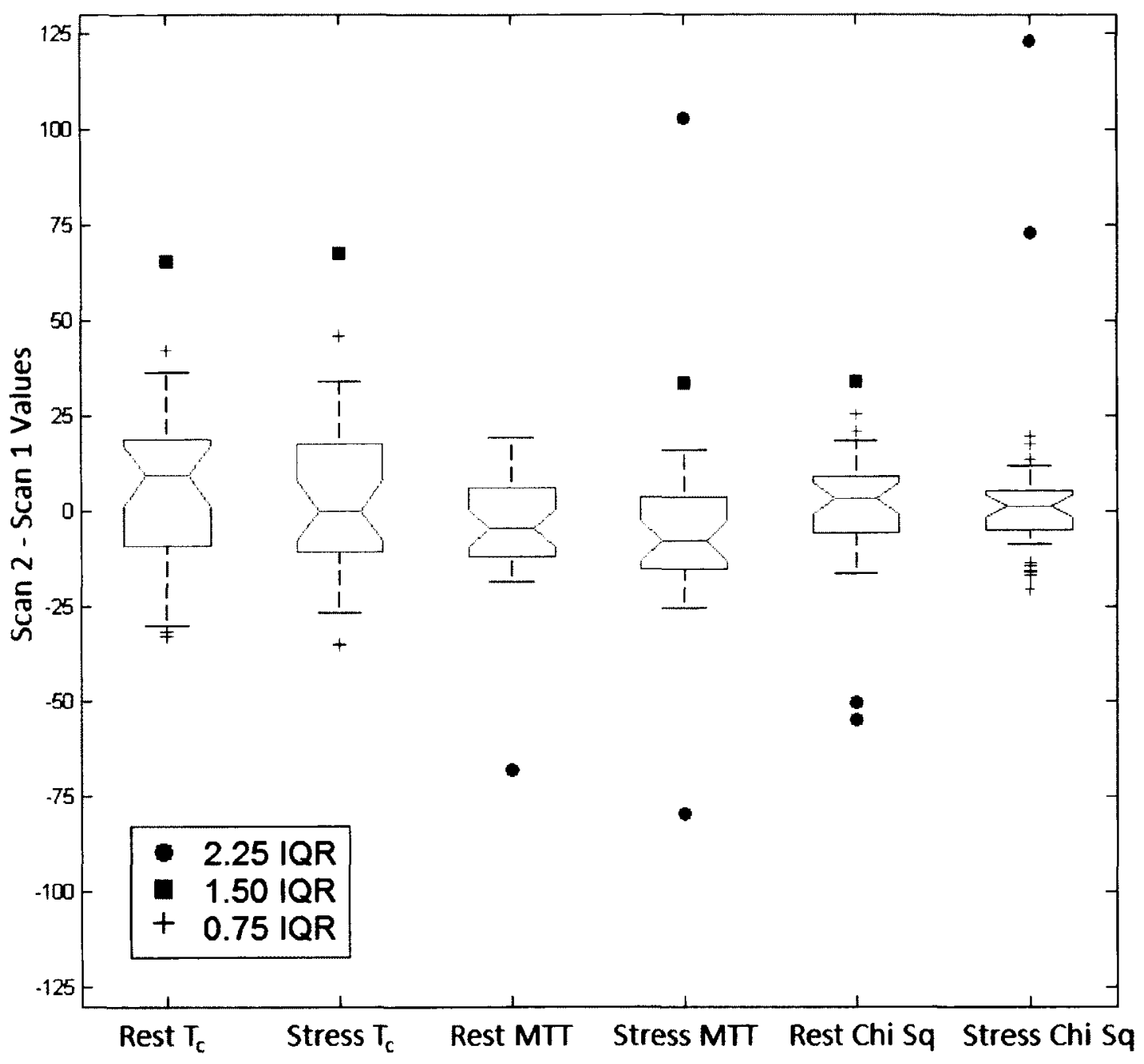

Figure 3.2: Notched box-plot comparison of infusion profile parameters extracted from the gamma variate function fit to the blood time-activity data. The labels $T_{c}, M T$, and Chi Sq represent clearance-time ( $s$ ), mean transit-time (s), and reduced Chi-squared respectively. Outliers at each exclusion level are noted with a unique symbol. 


\section{Hemodynamic Heterogeneity}

Differences in the RPP at rest and stress were compiled and grouped according to their distance from the median. Figure 3.3 shows box plots of rest, stress, and combined RPPs exclusion analyses with whisker limits extending to the last data point before 0.75 IQR. One subject was excluded as an outlier for rest at 2.25 IQR and the same subject was excluded at 1.5 IQR when rest and stress were combined. Three subjects were excluded at rest, four at stress, and seven in the combined RPP at 0.75 IQR.

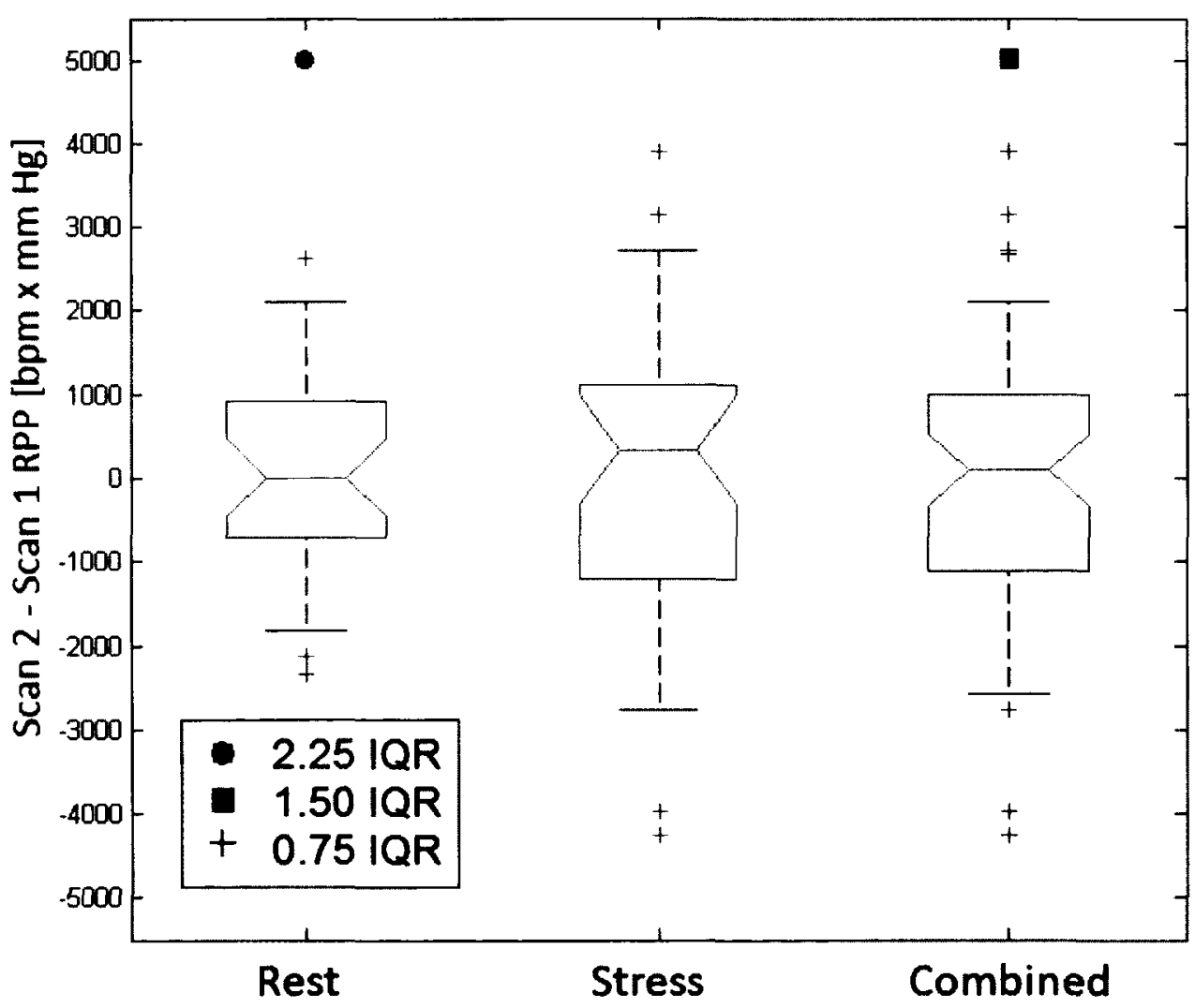

Figure 3.3: Hemodynamic parameter box-plot comparison of scan 2 minus scan 1 rate-pressure-product at rest, stress and combined. Outliers at each exclusion level are noted with a unique symbol. 


\subsection{Test-Retest Repeatability}

\subsubsection{Same-Day Study}

Table 3.7 shows the repeatability results for all rest protocol variations. The second phase introduced a cylindrical region of interest in the LA to sample the input function rather than the LV cavity ROI. The two most repeatable LV input rest MBF methods were analyzed with the LA input based on the Bland-Altman repeatability results (FBP-10-RPP and OSEM-6-SOC-RPP).

\section{Left Ventricle Input ROI}

Resting MBF values generally showed good correlation between repeat scans $\left(r^{2}=0.52-\right.$ $0.94)$, and were reduced after adjusting for changes in the RPP $\left(r^{2}=0.37-0.90\right)$. The OSEM-6-SOC-RPP protocol had the lowest RPC value $(0.27 \mathrm{~mL} / \mathrm{min} / \mathrm{g})$ and $\mathrm{CV}(18 \%)$ for LV methods, with no outliers greater than three standard deviations from the mean. The SOC tended to improve the RPC and CV for OSEM reconstructions but the opposite was observed for FBP.

Separating the OSEM-6-SOC results into normal and CAD patient groups, the RPC values were 0.20 and $0.31 \mathrm{~mL} / \mathrm{min} / \mathrm{g}$ respectively $(p=0.3)$. RPP-adjusted normal and CAD patient RPC values were 0.19 and $0.27 \mathrm{~mL} / \mathrm{min} / \mathrm{g}$ respectively $(p=0.7)$. The test-retest RPC was significantly smaller for reconstruction method OSEM-6-SOC vs. FBP-6-SOC ( $p=$ $0.005)$ and for spillover correction OSEM-6-SOC vs. OSEM-6 $(p=0.04)$, but not for scan 
Table 3.7: Same-day study test-retest repeatability of rest MBF measurements

\begin{tabular}{cclcccccccc}
\hline $\begin{array}{c}\text { Reconstruction } \\
\text { Method }\end{array}$ & $\begin{array}{c}\text { Scan } \\
\text { Time }\end{array}$ & $\begin{array}{c}\text { Input / } \\
\text { SOC }\end{array}$ & $\mathbf{r}^{2}$ & RPC & CV & Outlier & $\begin{array}{c}\mathbf{r}^{2} \\
\text { RPP }\end{array}$ & $\begin{array}{c}\text { RPC } \\
\text { RPP }\end{array}$ & $\begin{array}{c}\text { RP } \\
\text { RPP }\end{array}$ & $\begin{array}{c}\text { Outlier } \\
\text { RPP }\end{array}$ \\
\hline FBP & 10 & LV/SOC & 0.63 & 0.44 & 30 & 1 & 0.48 & 0.32 & 22 & 1 \\
FBP & 10 & LV & 0.78 & 0.33 & 20 & 1 & 0.65 & 0.26 & 16 & 0 \\
FBP & 6 & LV/SOC & 0.52 & 0.46 & 31 & 2 & 0.37 & 0.36 & 24 & 2 \\
FBP & 6 & LV & 0.67 & 0.36 & 21 & 0 & 0.50 & 0.30 & 18 & 0 \\
FBP & 10 & LA & 0.90 & 0.27 & 16 & 2 & 0.83 & 0.26 & 15 & 2 \\
OSEM & 10 & LV/SOC & 0.75 & 0.34 & 22 & 1 & 0.66 & 0.27 & 18 & 0 \\
OSEM & 10 & LV & 0.74 & 0.37 & 24 & 1 & 0.63 & 0.29 & 18 & 1 \\
OSEM & 6 & LV/SOC & 0.74 & 0.28 & 19 & 0 & 0.63 & 0.26 & 17 & 0 \\
OSEM & 6 & LV & 0.62 & 0.40 & 24 & 1 & 0.50 & 0.30 & 19 & 0 \\
OSEM & 6 & LA/SOC & $\mathbf{0 . 9 4}$ & $\mathbf{0 . 2 1}$ & $\mathbf{1 3}$ & $\mathbf{0}$ & $\mathbf{0 . 9 0}$ & $\mathbf{0 . 2 0}$ & $\mathbf{1 2}$ & $\mathbf{0}$ \\
\hline
\end{tabular}

Scan Time is in minutes, SOC = spillover correction, Input = blood input region for kinetic modeling, $r$ is Pearson's correlation, repeatability coefficient (RPC) is in $\mathrm{mL} / \mathrm{min} / \mathrm{g}$, coefficient of variation (CV) is MBF SD/mean $\times 100 \%$, outlier = data greater than three standard deviations from the mean, RPP = rate-pressure-product adjusted.

length or RPP adjustment ( $p>0.3$ ). Using the best LV protocol, OSEM-6-SOC, the RPC in males was significantly better than in females $(0.21 \mathrm{~mL} / \mathrm{min} / \mathrm{g} v \mathrm{vs} .0 .40 \mathrm{~mL} / \mathrm{min} / \mathrm{g} ; \mathrm{p}=$ $0.008)$ before applying the RPP adjustment but not after $(0.21 \mathrm{~mL} / \mathrm{min} / \mathrm{g}$ vs. 0.33 $\mathrm{mL} / \min . g ; p=0.07)$.

Figures 3.4 and 3.5 show the correlation and Bland-Altman plots of test-retest differences using the best LV protocol with and without the RPP adjustment, OSEM-6SOC. The plots also demonstrate the narrowed population range of mean MBF values after RPP-adjustment (Table 3.5), but with similar test-retest repeatability compared to the unadjusted values ( $R P C=0.26$ vs $0.28 ; p=0.7)$. 

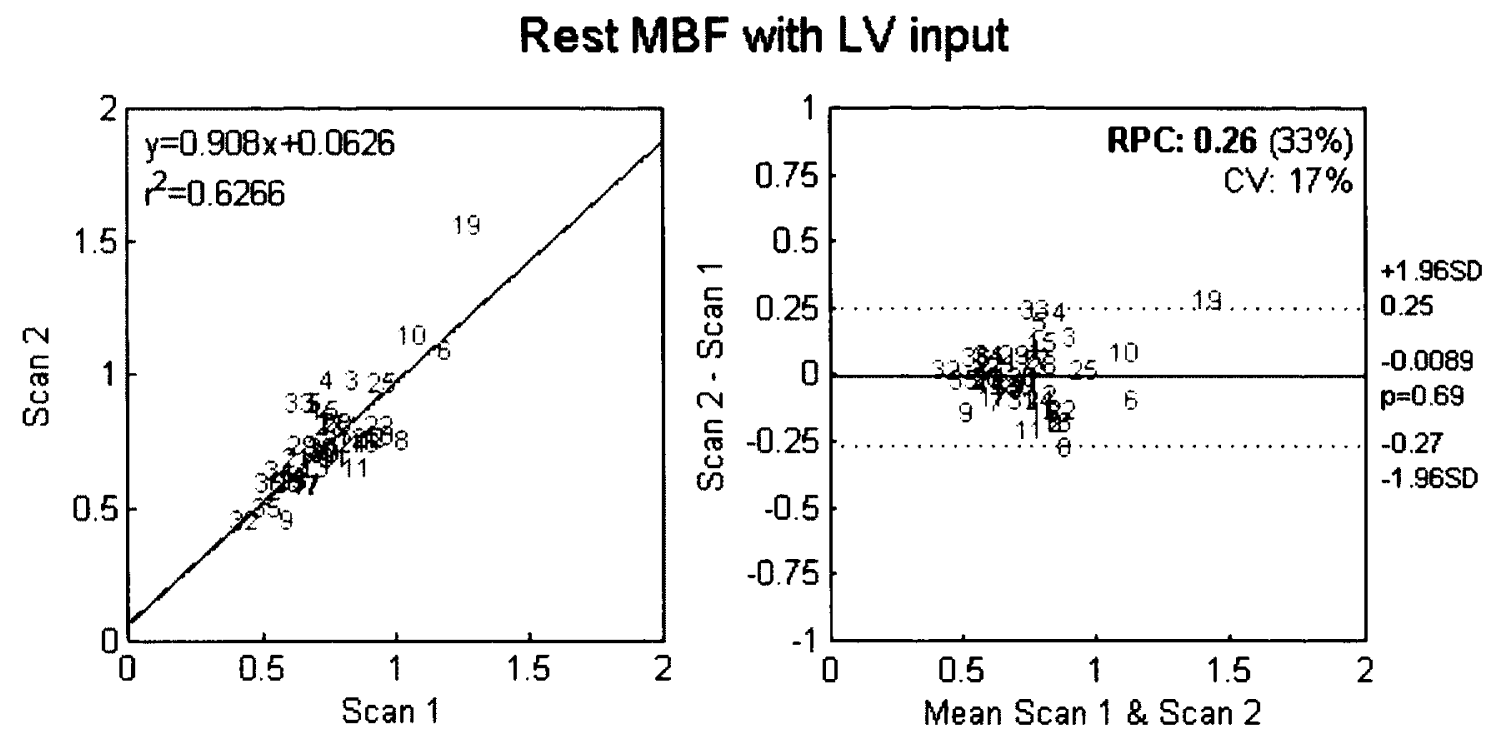

Figure 3.4: Correlation and Bland-Altman plots of test-retest differences in rest MBF values using the OSEM-6-SOC-RPP protocol with LV input.

\section{Left Atrium Input ROI}

The mean MBF using OSEM-6-LA-SOC-RPP $(\operatorname{scan} 1,0.86 \pm 0.34 \mathrm{~mL} / \mathrm{min} / \mathrm{g} ; \operatorname{scan} 2,0.86 \pm$ $0.32 \mathrm{~mL} / \mathrm{min} / \mathrm{g}$ ) was slightly higher compared to the LV input function (scan $1, p=0.02$; scan $2, p=0.008)$, but not significantly different than the original clinical protocol values (Table 3.5: FBP-10-RPP $=0.83 \mathrm{~mL} / \mathrm{min} / \mathrm{g}, \mathrm{p}>0.4$ ). The mean MBF using FBP-10-LA-RPP was also similar at $0.87 \pm 0.29 \mathrm{~mL} / \mathrm{min} / \mathrm{g}$.

Figure 3.6 shows the correlation and Bland-Altman results for the most repeatable rest protocol (OSEM-6-LA-SOC-RPP) overall. There were reductions in RPC $(0.21$ and 0.20 $\mathrm{mL} / \mathrm{min} / \mathrm{g})$ and CV (13\% and $12 \%)$ using both OSEM-6-LA-SOC and OSEM-6-LA-SOC-RPP compared to the LV cavity input function values shown in Figure 3.6 (RPP; $p=0.16$, 
unadjusted; $p=0.08)$. However, the RPC $(0.27$ and $0.26 \mathrm{~mL} / \mathrm{min} / \mathrm{g})$ and $\mathrm{CV}(16 \%$ and 15\%) using FBP-10 and FBP-10-RPP did not improve with the LA compared to the LV cavity input function values.

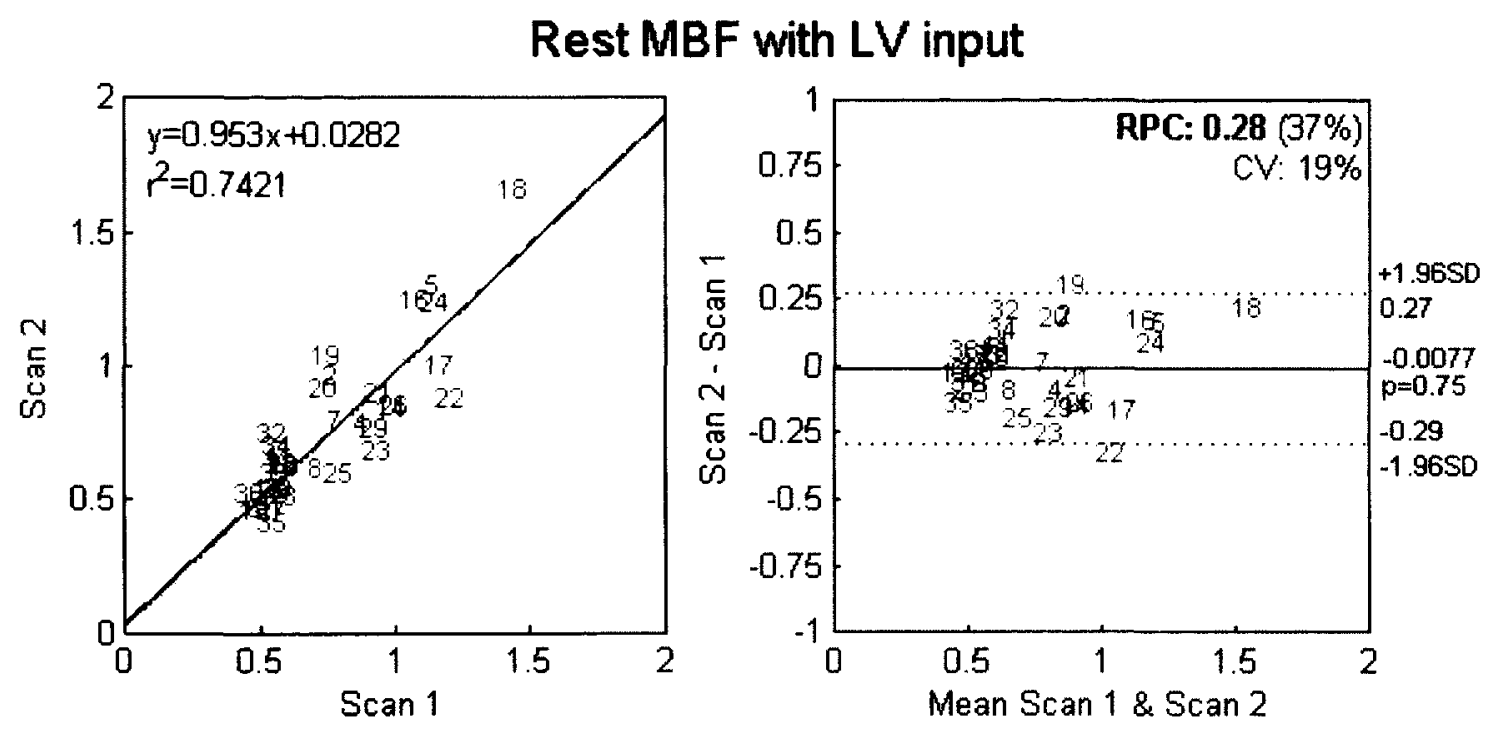

Figure 3.5: Correlation and Bland-Altman plots of test-retest differences in rest MBF values using the OSEM-6-SOC protocol with LV input. 

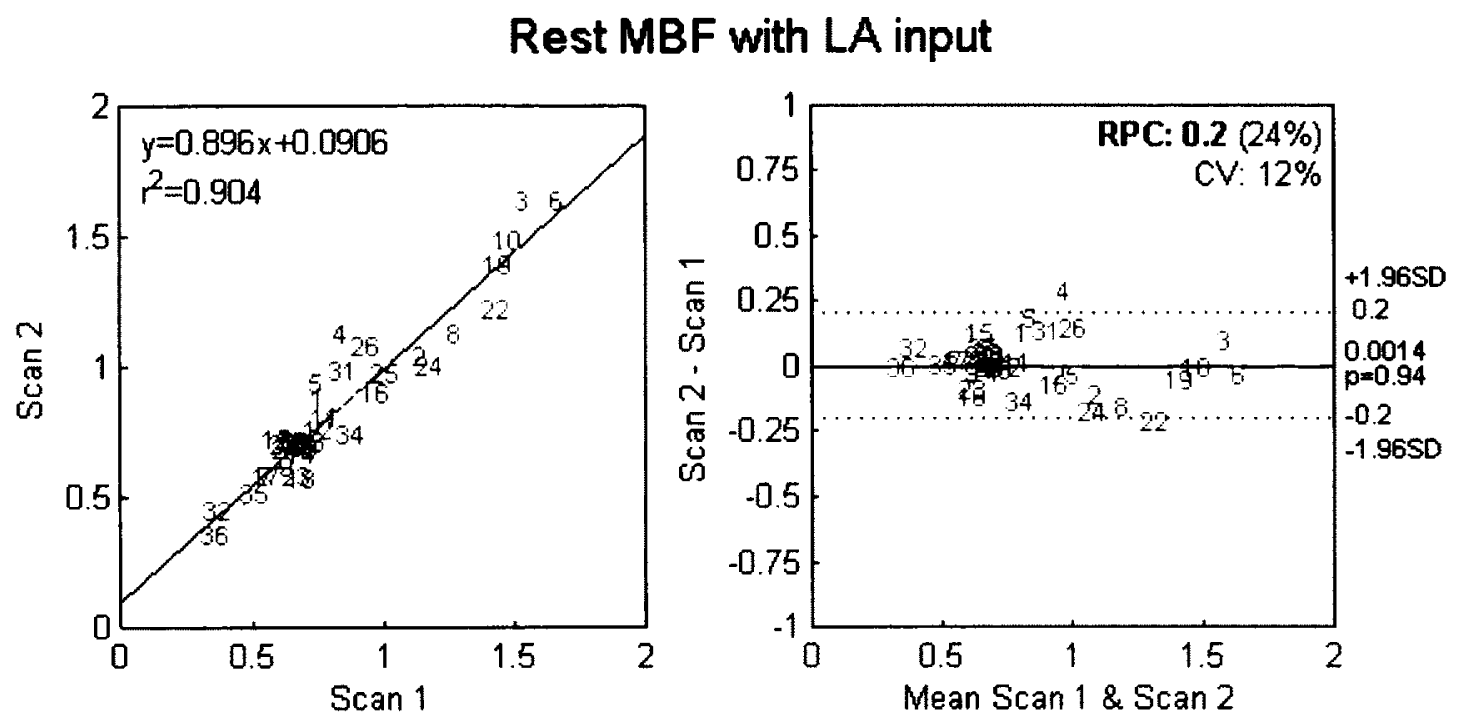

Figure 3.6: Correlation and Bland-Altman plots of test-retest differences in rest MBF values using the OSEM-6-SOC-RPP protocol with LA input.

Table 3.8 shows the repeatability results of the single stress scan MFR with the most repeatable rest protocols. Similar to the rest MBF results, the most repeatable MFR results were obtained using OSEM-6-LA-SOC-RPP $(\mathrm{RPC}=0.5)$ as expected. The testretest variance was significantly better than the next most repeatable protocol FBP-10LA ( $p=0.04)$. Figure 3.7 shows the MFR correlation and Bland-Altman plots using the optimal OSEM-6-LA-SOC-RPP protocol. 
Table 3.8: Same-day study test-retest repeatability of myocardial flow reserve (MFR)†

\begin{tabular}{cccccccccc}
\hline & & & \multicolumn{3}{c}{ MFR } & \multicolumn{3}{c}{ MFR RPP } \\
\hline $\begin{array}{c}\text { Reconstruction } \\
\text { Method }\end{array}$ & $\begin{array}{c}\text { Scan } \\
\text { Time }\end{array}$ & $\begin{array}{c}\text { Input / } \\
\text { SOC }\end{array}$ & $\mathbf{r}^{2}$ & RPC & CV & $\begin{array}{c}\mathbf{r}^{2} \\
\text { RPP }\end{array}$ & $\begin{array}{c}\text { RPC } \\
\text { RPP }\end{array}$ & $\begin{array}{c}\text { RP } \\
\text { RPP }\end{array}$ \\
\hline FBP & 10 & LV & 0.84 & 0.83 & 16 & 0.84 & 0.85 & 17 \\
FBP & 10 & LV / SOC & 0.84 & 0.83 & 15 & 0.73 & 1.0 & 19 \\
FBP & 10 & LA & 0.87 & 0.75 & 14 & 0.96 & 0.88 & 15 \\
OSEM & 6 & LV / SOC & 0.80 & 0.92 & 18 & 0.81 & 0.85 & 17 \\
OSEM & 6 & LV & 0.73 & 1.00 & 22 & 0.77 & 1.00 & 22 \\
OSEM & 6 & LA /SOC & $\mathbf{0 . 9 1}$ & $\mathbf{0 . 5 8}$ & $\mathbf{1 2}$ & $\mathbf{0 . 9 0}$ & $\mathbf{0 . 5 2}$ & $\mathbf{1 1}$ \\
\hline
\end{tabular}

† A single stress scan paired with repeated rest scans.

Myocardial flow reserve (MFR) is unit-less ratio of stress/rest MBF, RPP = rate-pressure-product adjusted, Time is in minutes, SOC = spillover correction, Input = blood input

$r^{2}$ is Pearson's correlation, Repeatability coefficient (RPC) is in $\mathrm{mL} / \mathrm{min} / \mathrm{g}$

Coefficient of variation (CV) is MFR SD/mean $\times 100 \%$

${ }^{*} p=0.04$ vs. FBP-10 protocol with LA input

\section{MFR with LA input}
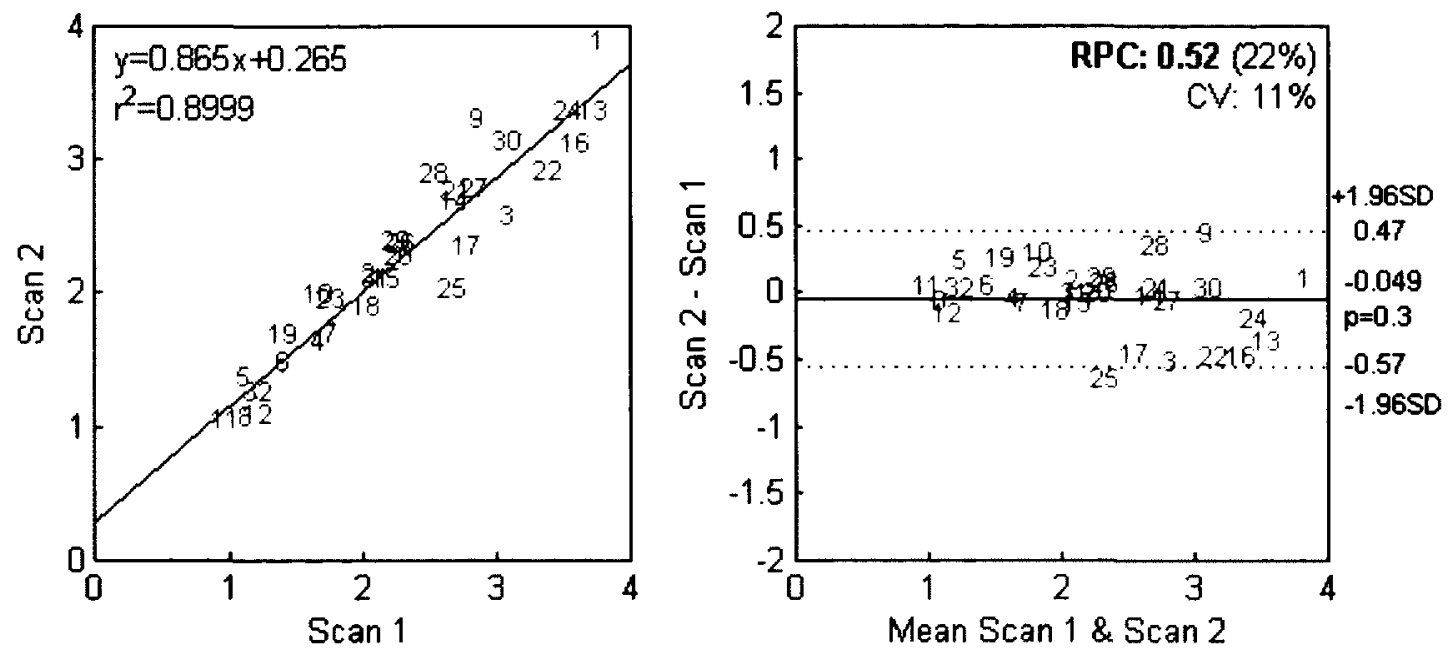

Figure 3.7: Correlation and Bland-Altman plots of test-retest differences in MFR values using the OSEM-6-LA-SOC-RPP protocol. 


\subsubsection{Serial Study}

\section{Effect of Infusion Profile and Hemodynamic Heterogeneity}

Table 3.9 shows the repeatability results for the three infusion profile and hemodynamic exclusion levels. The exclusion of heterogeneous profiles significantly improved the repeatability of stress scans at all levels $(p<0.03)$. The RPC values for all rest and stress flow values were lowest at 1.5 IQR with the eight most heterogeneous infusion profile subjects excluded. RPC values for MFR and adjusted MFR were slightly lower at 1.5 IQR.

Repeatability assessed with hemodynamic exclusion criteria showed a trend similar to the infusion profile results. The RPCs for all flow values were lowest at 0.75 IQR with seven of the most hemodynamically heterogeneous subjects excluded, whether outliers were evaluated at rest and stress separately or combined. Combining the rest and stress RPP exclusion parameters marginally improved the repeatability vs. separate RPP exclusion for all values.

There were nine total subjects excluded using the 1.5 IQR combined infusion profile and hemodynamic criteria. One subject was excluded by two infusion profile exclusion categories. The resulting population $(n=22)$ mean MBF at rest and adjusted rest were $0.66 \pm 0.23 \mathrm{~mL} / \mathrm{min} / \mathrm{g}$ and $0.67 \pm 0.21 \mathrm{~mL} / \mathrm{min} / \mathrm{g}$ respectively for scan $1(p=0.8)$ and $0.64 \pm 0.22 \mathrm{~mL} / \mathrm{min} / \mathrm{g}$ and $0.67 \pm 0.22 \mathrm{~mL} / \mathrm{min} / \mathrm{g}$ respectively for scan $2(p=0.8)$. Stress 
Table 3.9: Test-retest repeatability at rest and stress MBF, and MFR for different infusion profile and hemodynamic exclusion criteria

\begin{tabular}{cccccccccccc} 
Group & \multicolumn{2}{c}{ Rest } & \multicolumn{2}{c}{ Rest RPP } & \multicolumn{2}{c}{ Stress } & \multicolumn{2}{c}{ MFR } & \multicolumn{2}{c}{ MFR RPP } \\
\hline Infusion Profile & RPC & $\%$ & RPC & $\%$ & RPC & $\%$ & RPC & $\%$ & RPC & $\%$ \\
\hline Population $(n=31)$ & 0.38 & 56 & 0.28 & 41 & 1.40 & 84 & 1.30 & 51 & 1.50 & 62 \\
\hline 2.25 IQR $(n=26)$ & 0.33 & 51 & 0.23 & 36 & $0.81^{\mathrm{a}}$ & 52 & 1.20 & 48 & $1.30^{\mathrm{b}}$ & 53 \\
1.50 IQR $(n=23)$ & 0.26 & 40 & 0.22 & 33 & $0.67^{a}$ & 41 & 1.30 & 48 & 1.30 & 52 \\
0.75 IQR $(n=15)$ & 0.26 & 41 & 0.25 & 39 & $0.80^{\mathrm{a}}$ & 49 & 1.40 & 51 & 1.60 & 59 \\
\hline
\end{tabular}

\begin{tabular}{ccccccccccc} 
Hemodynamic & \multicolumn{2}{c}{ Rest } & \multicolumn{3}{c}{ Rest RPP } & \multicolumn{2}{c}{ Stress } & \multicolumn{2}{c}{ MFR } & \multicolumn{2}{c}{ MFR RPP } \\
\hline Separate & RPC & $\%$ & RPC & $\%$ & RPC & $\%$ & RPC & $\%$ & RPC & $\%$ \\
\hline 2.25 IQR $(n=30)$ & 0.38 & 56 & 0.28 & 41 & 1.40 & 83 & 1.30 & 51 & 1.40 & 58 \\
1.50 IQR $(n=30)$ & 0.38 & 56 & 0.28 & 41 & 1.40 & 83 & 1.30 & 51 & 1.40 & 58 \\
0.75 IQR $(n=24)$ & 0.31 & 46 & 0.28 & 40 & $1.00^{2}$ & 62 & 1.20 & 49 & 1.30 & 53 \\
\hline Combined & & & & & & & & & & \\
\hline 2.25 IQR $(n=31)$ & 0.38 & 56 & 0.28 & 41 & 1.40 & 84 & 1.30 & 51 & 1.50 & 62 \\
1.50 IQR $(n=30)$ & 0.38 & 56 & 0.28 & 41 & 1.40 & 83 & 1.30 & 51 & 1.40 & 58 \\
0.75 IQR $(n=24)$ & 0.30 & 45 & 0.26 & 38 & $0.97^{a}$ & 59 & 0.98 & 35 & 1.00 & 43 \\
\hline
\end{tabular}

\begin{tabular}{|c|c|c|c|c|c|c|c|c|c|c|}
\hline \multirow{2}{*}{$\begin{array}{c}1.5 \text { IQR } \\
\text { Infusion Profile + }\end{array}$} & \multicolumn{2}{|c|}{ Rest } & \multicolumn{2}{|c|}{ Rest RPP } & \multicolumn{2}{|c|}{ Stress } & \multicolumn{2}{|c|}{ MFR } & \multicolumn{2}{|c|}{ MFR RPP } \\
\hline & RPC & $\%$ & RPC & $\%$ & RPC & $\%$ & RPC & $\%$ & RPC & $\%$ \\
\hline 1.50 Hemo $(n=22)+$ & 0.26 & 40 & 0.21 & 32 & $0.65^{a}$ & 40 & 1.30 & 48 & 1.10 & 45 \\
\hline Excluded $(n=9)$ & $0.58^{d}$ & 78 & $0.41^{d}$ & 58 & $2.50^{d}$ & 140 & 1.40 & 62 & $2.30^{d}$ & 98 \\
\hline 0.75 Hemo $(n=18)+$ & 0.27 & 41 & 0.19 & 28 & $0.53^{c}$ & 32 & $0.78^{c}$ & 29 & $0.95^{c}$ & 37 \\
\hline Excluded $(n=13)$ & $0.50^{e}$ & 70 & $0.38^{e}$ & 54 & $2.10^{\mathrm{e}}$ & 120 & $1.80^{\mathrm{e}}$ & 78 & $2.00^{\mathrm{e}}$ & 90 \\
\hline $\begin{array}{l}\text { MFR = myocardial fl } \\
\%=R P C \text { as a percen } \\
\text { RPP = rate-pressure } \\
\text { Separate = rest and } \\
\text { Combined = rest an } \\
+ \text { Combined hemod } \\
\text { a } p<0.03 \text { vs. popula } \\
{ }^{c} p<0.05 \text { vs. popula } \\
\text { e } p<0.05 \text { vs. } 0.75 \mathrm{H}\end{array}$ & $\begin{array}{l}\text { W rese } \\
\text { of the } \\
\text { produc } \\
\text { tress } R \\
\text { stress } \\
\text { namic } \\
\text { ion stre } \\
\text { ion valt }\end{array}$ & $\begin{array}{l}\text { Ie, R } \\
\text { lean } \\
\text { adjus } \\
\text { P exc } \\
\text { PP va } \\
\text { sclus } \\
s^{b}\end{array}$ & $\begin{array}{l}\text { peatab } \\
\text { low } \\
\text { ted, IQF } \\
\text { usions } \\
\text { lues cor } \\
\text { ons use } \\
=0.02 \\
<0.02 \\
\text { roup }\end{array}$ & $\begin{array}{l}\text { ty co } \\
=\text { inte } \\
\text { ylua } \\
\text { bine } \\
\text { in ac } \\
\text { po }\end{array}$ & $\begin{array}{l}\text { fficien } \\
\text { eduartil } \\
\text { ed sep } \\
\text { to det } \\
\text { dition } t \\
\text { ulation }\end{array}$ & $\begin{array}{l}\text { (RPC) } \\
\text { rang } \\
\text { rately } \\
\text { rmine } \\
\text { the } 1 \\
\text { UFR R }\end{array}$ & $\begin{array}{l}\text { exclusic } \\
50 \text { IQR } \\
\text { P }\end{array}$ & $\begin{array}{l}\text { ns } \\
\text { nfus }\end{array}$ & on Prof & \\
\hline
\end{tabular}


flow remained significantly increased compared to rest at $1.66 \pm 0.60 \mathrm{~mL} / \mathrm{min} / \mathrm{g}$ for scan 1 and $1.61 \pm 0.67 \mathrm{~mL} / \mathrm{min} / \mathrm{g}$ for scan $2(p<0.001)$. The combined rest and stress RPC was significantly larger for the whole population vs. the 1.5 IQR combined inclusion group $(1.0 \mathrm{~mL} / \mathrm{min} / \mathrm{g}$ vs. $0.49 \mathrm{~mL} / \mathrm{min} / \mathrm{g} ; \mathrm{p}<0.001)$. Myocardial flow reserve and adjusted MFR for scan 1 were $2.66 \pm 0.86$ and $2.50 \pm 0.65$ respectively and for scan 2 were $2.63 \pm 1.02$ and $2.50 \pm 0.93$ respectively. There were no significant differences in scan 1 vs. scan 2, RPP adjusted vs. unadjusted, or included sub-group vs. whole population flow values ( $p=N S$ ). With the combined hemodynamic and tracer infusion profile exclusion criteria applied, the stress flow had a significantly smaller RPC than the whole population value $(p=0.003)$. Almost all the included sub-group rest, stress, and MFR values were significantly more repeatable than the values of the corresponding excluded group.

A combination of the 1.5 IQR infusion profile exclusion and the 0.75 IQR combined hemodynamic exclusion criteria provides some addition insight into the effect of hemodynamic heterogeneity on repeatability. Thirteen total subjects were excluded; two subjects were in both exclusion groups. When these two exclusion groups were combined, the resulting population MBF mean at rest and adjusted rest values were $0.66 \pm 0.23 \mathrm{~mL} / \mathrm{min} / \mathrm{g}$ and $0.68 \pm 0.23 \mathrm{~mL} / \mathrm{min} / \mathrm{g}$ respectively for scan 1 and $0.64 \pm 0.23$ $\mathrm{mL} / \mathrm{min} / \mathrm{g}$ and $0.67 \pm 0.23 \mathrm{~mL} / \mathrm{min} / \mathrm{g}$ respectively for scan 2 . Stress flow remained significantly increased compared to rest at $1.68 \pm 0.65 \mathrm{~mL} / \mathrm{min} / \mathrm{g}$ for scan 1 and $1.66 \pm$ $0.66 \mathrm{~mL} / \mathrm{min} / \mathrm{g}$ for scan $2(p<0.001)$. The RPC of the combined rest and stress MBF was 
$0.43 \mathrm{~mL} / \mathrm{min} / \mathrm{g}$. Myocardial flow reserve and adjusted MFR for scan 1 were $2.64 \pm 0.82$ and $2.50 \pm 0.66$, and for scan 2 were $2.73 \pm 0.97$ and $2.58 \pm 0.91$ respectively $(p=N S)$. Scan 1 vs. scan 2, RPP adjusted vs. unadjusted, and included sub-group vs. whole population flow values were not significantly different $(p>0.05)$, as expected. With these combined exclusion criteria, the stress flow, MFR, and adjusted MFR again had significantly smaller RPCs than the whole population values $(p<0.05)$. All of the included sub-group flow values were significantly more repeatable than the values of the excluded group.

\subsubsection{Study Comparison}

Figure 3.8 shows a repeatability comparison between the same-day study and the serial study.

The RPC typically decreases as the scans become less heterogeneous in respect to RPP differences and tracer infusion profile differences. 


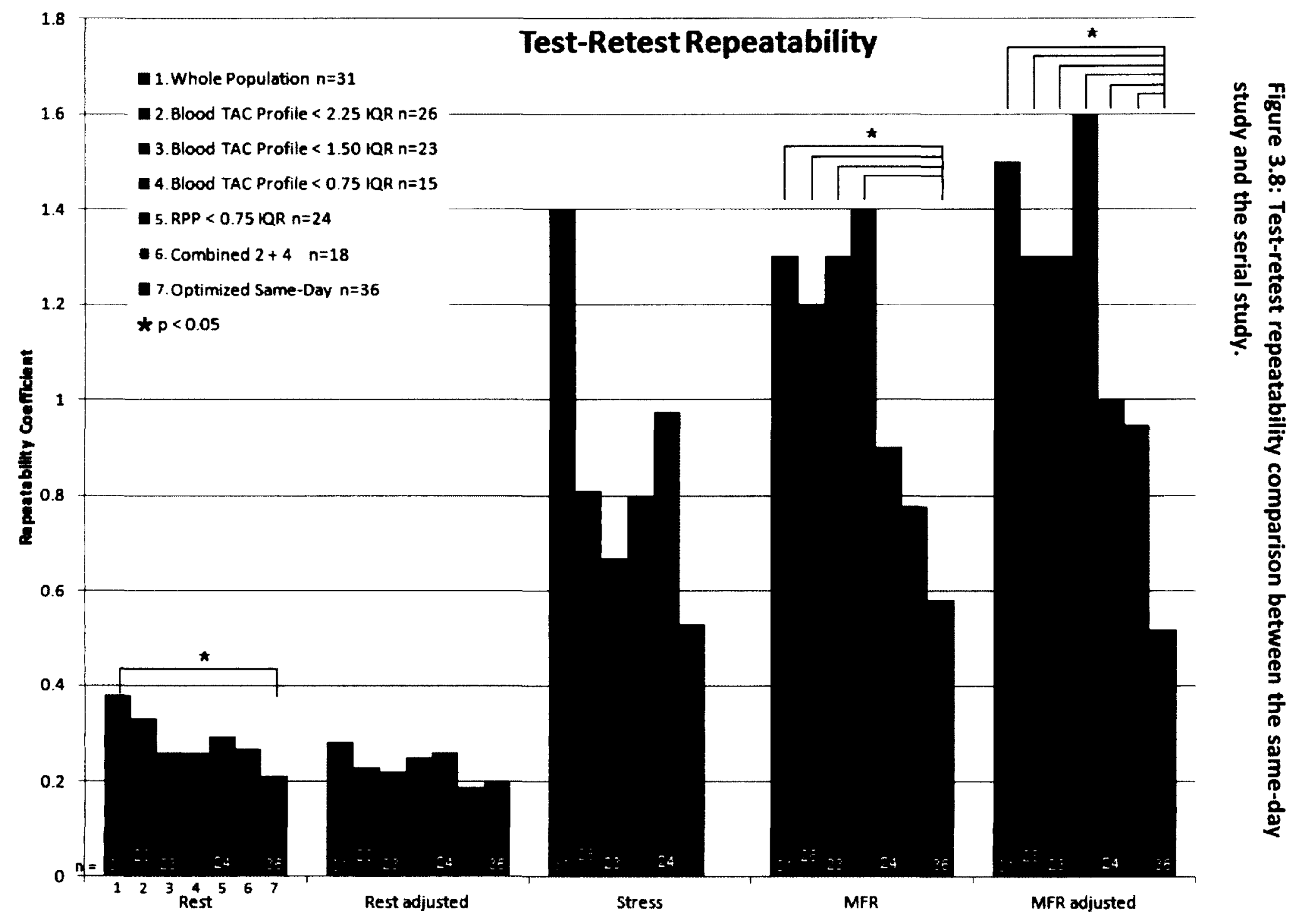




\section{Chapter 4: Discussion}

The goal of this research was to investigate the test-retest repeatability of global left ventricle MBF and MFR using ${ }^{82}$ Rb PET imaging. In the first study, the optimal same-day test-retest repeatability protocol parameters were investigated at rest. The protocol was optimized by investigating the effects of scan length, image reconstruction method, dual-spillover correction, RPP adjustment, gender, location of blood input function, and CAD on mean blood flow, population variance, and test-retest repeatability. In the second study, the test-retest repeatability effects of heterogeneous hemodynamic conditions and tracer infusion profiles on MBF and MFR were investigated.

\subsection{Reconstruction}

Statistical iterative reconstruction methods such as OSEM used in the present study have become the de facto standard for relative MPI with PET. However, there are some important non-linear effects that can potentially limit the accuracy of MBF quantification using OSEM or other statistical-based methods. Compared to analytic methods such as FBP that have a stable and predictable linear behavior, the reconstructed resolution and contrast recovery with OSEM can vary spatially and dynamically, depending on the local count statistics and convergence properties of the particular algorithm implementation. The accuracy of MBF measurements using a particular imaging instrument, iterative reconstruction code, and tracer kinetic analysis 
should always be validated for the intended use against an appropriate gold-standard (30) (31) (64).

The optimal same-day protocol included the iterative OSEM reconstruction method. Several other studies have investigated the effects of iterative reconstruction on quantitative MBF accuracy. The underestimation of MBF by OSEM has been shown in pig studies with $3 \mathrm{D} \mathrm{H}_{2}{ }^{15} \mathrm{O}$ PET vs. microspheres (70), in patients with $2 \mathrm{D}{ }^{13} \mathrm{NH}_{3}$ PET vs. FBP (71), and ${ }^{13} \mathrm{NH}_{3}$ PET vs. FBP (72), but not in patients with $2 \mathrm{D}^{18}$ F-FDG PET vs. FBP (73) or in $3 D{ }^{18}$ F-FDG PET vs. FBP (74). Aggressive smoothing of OSEM images was cited as a common factor in underestimating MBF. Different combinations of iterations and subsets were studied but none were the same as the four iteration, 24 subset protocol used in the present studies. The same-day study did not result in a systematic decrease in MBF when comparing OSEM to FBP reconstructions (Table 3.5).

\subsection{Scan Length}

A number of the same-day myocardial TACS showed apparent increasing blood activity in the last four-minute frame of the ten-minute scan. This included points sampled in the LV myocardium, base, atrium, and cavity. Shortening the scan time from ten to six minutes appeared to eliminate the noisy blood pool and myocardium signal observed at this late time-point. Shortening the scan could also reduce the likelihood or severity of patient motion, which can be more common in the final four-minute time-frame. The 
results of the same-day study show that a six- vs. ten-minute scan improved repeatability.

Most patients undergo a rest and stress scan in the same imaging session. A $40 \%$ reduction in PET scan time (ten to six minutes) could reduce the time patients spend on the scanner couch and improve their overall experience. The time between rest and stress scans could thus be shortened, still allowing for full decay of the rest dose, as well as the time after the stress scan.

The data from healthy volunteer number 30 were removed from the serial study because the peak activity coincided with the scan start time. It is known that the tracer kinetic model is not able to accurately represent the system without the early blood information. A large number of TACs (30 out of 128 scans) showed that the recording of PET data started 10 to 30 seconds after the initial appearance of activity. Since the peak in blood activity was typically observed at 40 to 45 seconds for both rest and stress, a small portion of the early data was often missing, likely contributing to the test-retest variability. Clinical measures were implemented subsequently to ensure a shorter delay between tracer injection and the start of data acquisition.

Patient body motion is a potential concern between and during scans. To minimize radiation dose in the same-day study, a single rest CT attenuation correction scan was used to correct both rest ${ }^{82} \mathrm{Rb}$ PET scans. Patient translational motion $(x, y, z)$ between scans was corrected manually using the standard ACQC PET-CT alignment and 
attenuation correction software. Small body movements within scans (e.g., between dynamic frames) were observable in a majority of cases, mostly in the final four-minute frame that was excluded in the optimal six-minute scan protocol. Intra-scan motion correction was not one of the goals of this study, but it is important to recognize that patient body and internal organ motion can worsen the repeatability and accuracy of absolute MBF quantification.

\subsection{Blood Input Function}

The optimal protocol included an input function located in the LA cavity. Hove et al (61) studied the use of an LA input function as a way to circumvent the spillover of activity from the surrounding left ventricular walls. Their study found no bias in MBF using the LA vs. the LV input function. The present same-day study also detected no MBF bias between FBP-LA, FBP-LV, FBP-LV-SOC, OSEM-LA-SOC, or OSEM-LV input functions ( $p=$ NS), but OSEM-LA-SOC vs. OSEM-LV-SOC showed a trend towards a decrease of $\sim 0.1$ $\mathrm{mL} / \mathrm{min} / \mathrm{g}$ in resting $\mathrm{MBF}(\mathrm{p}=0.11$ and $0.052 ;$ scan 1 and scan 2$)$ and $\mathrm{RPP}$ adjusted MBF $(p=0.052$ and $0.02 ;$ scan 1 and scan 2$)$. This suggests that the SOC and LV input function together may underestimate MBF. Lubberink et al reported better accuracy in estimating image-derived input functions with $2 \mathrm{D}$ or $3 \mathrm{D}{ }^{18} \mathrm{~F}$-FDG PET using the ascending aorta, which, like the LA, is not associated with myocardial spillover contamination in the late blood data, but may be more sensitive to motion artifacts (74). 
Both present studies used a 30 -second constant-activity-rate uniform infusion of ${ }^{82} \mathrm{Rb}$ to optimize test-retest repeatability, and to limit PET system dead-time losses during the tracer first-pass through the heart and lungs. Previous investigations suggest that the precision of MBF estimates may be further improved by using even longer infusion intervals, e.g., one to four minutes, together with the one-tissue-compartment model (58). This type of slow-bolus controlled infusion may be particularly advantageous for MBF imaging on 3D PET systems with limited dynamic range, where it is essential to maintain quantitative accuracy at the peak count-rates encountered during the firstpass transit of activity through the circulatory system. Future investigations might help to determine potential trade-offs between accuracy and precision, using protocols optimized for absolute MBF quantification vs. relative MPI for routine clinical use.

\subsection{Spillover Correction}

Spillover from the myocardium into the blood pool, which contaminates the LV blood input function and might cause an underestimation in measured MBF, is a concern in flow quantification. In the same-day study the SOC negatively biased MBF measurements (Table 3.5) compared to clinical values but improved repeatability (Tables 3.7, 3.8). The introduction of the LA input function removed the MBF bias at rest. Since the LA region was not investigated without the SOC, it was not possible to determine whether the SOC may be unnecessary when using the LA input. Recently, Katoh et al (75) showed that a global spillover correction and an LV input improved 
inter-operator repeatability and improved stability in estimating regional MBF, similar to the same-day study.

\subsection{Hemodynamics}

Hemodynamic differences in the same-day study between males and females were associated with significant changes in resting $M B F$ measurements $(\sim 0.3 \mathrm{~mL} / \mathrm{min} / \mathrm{g})$. After adjusting scan $1 \mathrm{MBF}$ values for these differences in the rest RPP, the mean change between male and female subjects was no longer significant as shown in Figure 3.1. However, when scan 1 and scan 2 data were pooled, a significant gender difference of $0.1 \mathrm{~mL} / \mathrm{min} / \mathrm{g}$ remained even after applying the RPP adjustment. Previous studies have reported that myocardial spillover effects (e.g., FBV) may bias PET measurements of MBF (48). The size of the LV cavity, thickness of the myocardium, and therefore the variable activity spillover between blood pool and myocardium, could potentially affect the kinetic modeling accuracy of MBF measurements in men vs. women. In this study male and female subjects did have slightly different fractional blood volume (FBV $=0.33$ vs. $0.37 ; p<0.001$ ), and LV short-axis dimension ( 56.4 vs. 46.8 millimeters; $p<0.001$ ), but the myocardium-to-blood spillover fractions $(\boldsymbol{\beta})$ were not significantly different between genders $(0.86$ vs. $0.87 ; p>0.5)$. Adjustment of the MBF values for differences in FBV, in addition to RPP, eliminated the residual gender difference in MBF 10.77 $\mathrm{mL} / \mathrm{min} / \mathrm{g}$ mean value in males and females), suggesting that a small bias in estimation of the FBV may account for the residual differences in MBF. Interestingly, an inverse 
adjustment for LV dimension (MBF $\times$ individual subject LV dimension $\div$ population average LV dimension), in addition to RPP, also reduced the residual gender difference to $\sim 0.05 \mathrm{~mL} / \mathrm{min} / \mathrm{g}(p=0.24)$, suggesting that a small physiologic effect of heart size on MBF may also be possible.

Adjusting the individual MBF values to the population average RPP reduced the population variance significantly (Table 3.5 ). In the same-day study, the normal range of resting MBF was influenced heavily by a single patient with a very high resting RPP. Following adjustment for the individual scan-specific RPP, this resting MBF outlier was corrected to a value within the population mean \pm 2 SD. Similarly, the test-retest RPP adjusted RPC and CV showed incremental improvement compared to unadjusted values. In the serial study, RPP adjustment showed a decrease in test-retest RPC vs. unadjusted RPC at rest (Table 3.9), but the opposite was true for MFR, which showed no change, or an increase, in RPP adjusted RPC vs. RPC. Previous studies (30) (54) (56) (57) have found an increase in resting population variance and RPC with RPP adjustment. However, Manabe (57) reported that the RPP adjustment decreased the test-retest repeatability at rest, similar to the results of the present study.

Figures 4.1 (subject number 27) and 4.2 (subject number 18) show example TACs with similar blood curves in repeated scans, comparing the effect of a similar RPPs vs. very different RPPs on MBF. The MBF of the subject with similar RPP conditions is much more repeatable than the MBF of the subject with the large difference. This suggests that 
measurements of heart rate and blood pressure must be performed carefully to avoid introducing additional variability into the adjusted MBF values. In the present studies resting heart rate and blood pressure measurements were performed immediately before and after tracer injection, and the average values used to calculate the scanspecific RPP. The stress hemodynamic data were taken at the time of the peak heart rate during the scan to calculate the RPP.
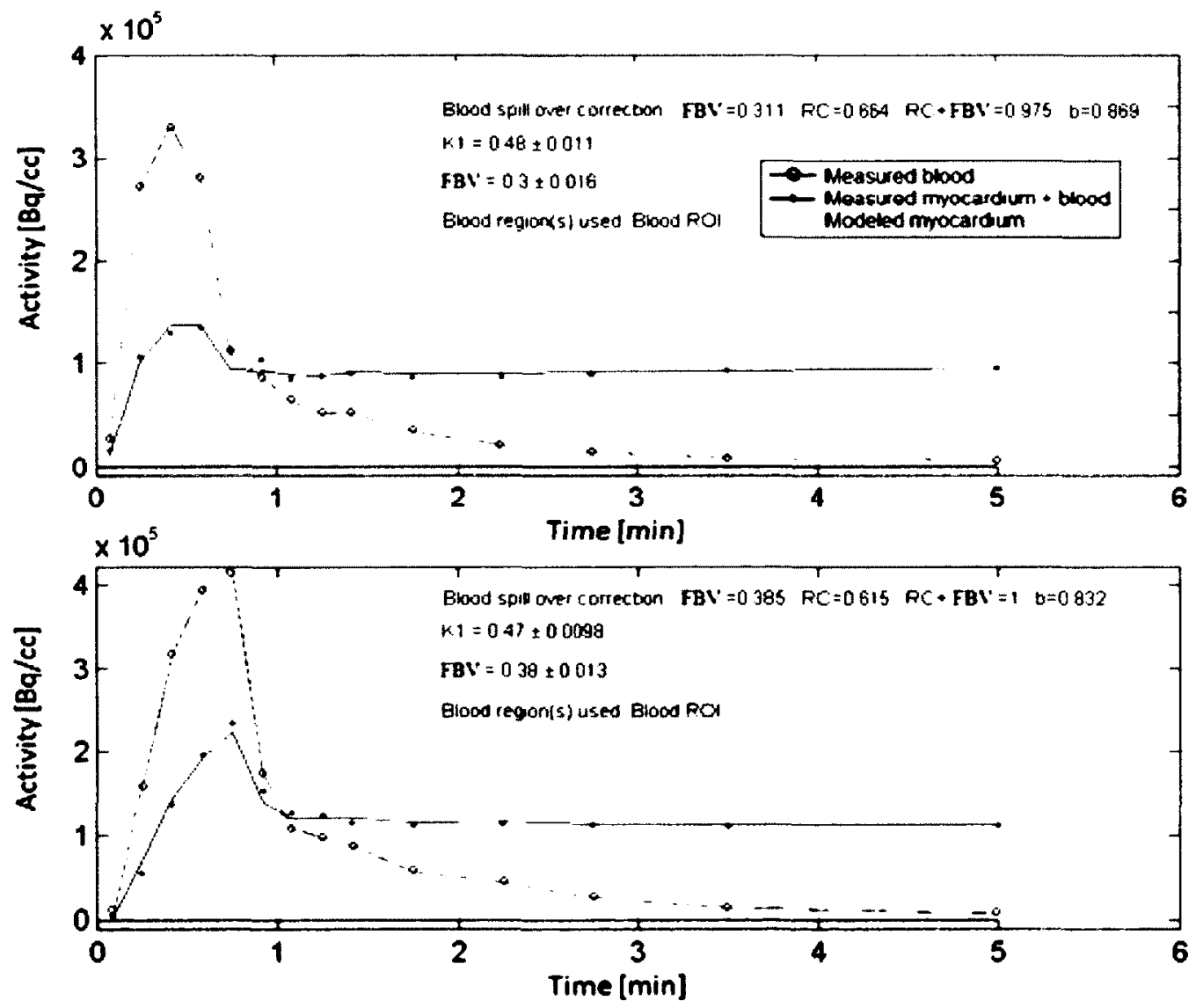

Figure 4.1: Time activity curves showing similar blood curves ( $\triangle \mathrm{FBV}=$ $0.08)$, similar rest $R P P$ values $(\triangle R P P=150)$, and similar MBFs $(\triangle M B F=$ 0.00 ; scan 1 and $2=0.58 \mathrm{~mL} / \mathrm{min} / \mathrm{g})$. 

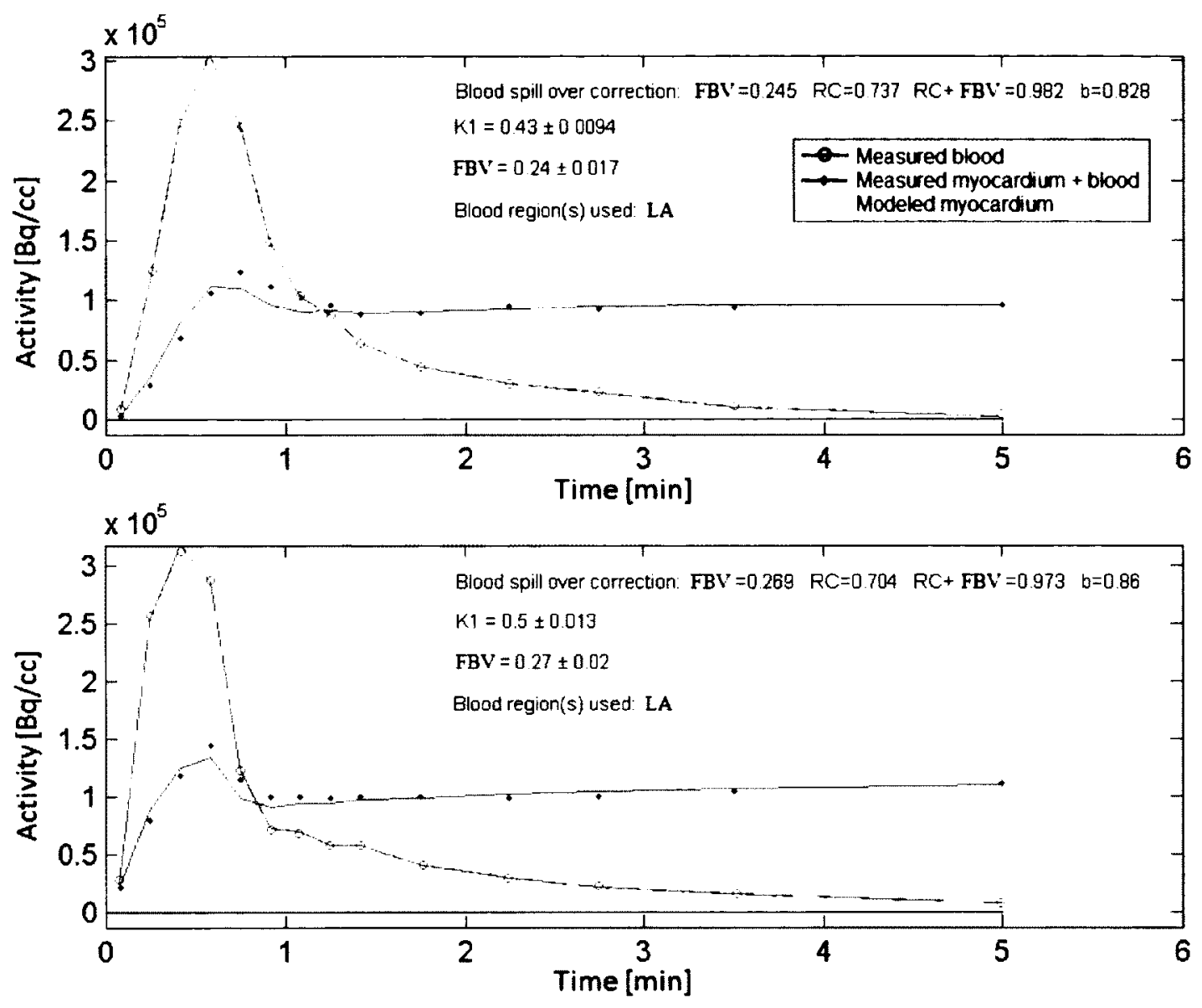

Figure 4.2: Time activity curves showing similar blood curves ( $\triangle \mathrm{FBV}=$ 0.03), different rest $R P P$ values ( $\triangle R P P=1390$ ), and different MBFs ( $\triangle M B F=0.27 ; \operatorname{scan} 1=0.50, \operatorname{scan} 2=0.77 \mathrm{~mL} / \mathrm{min} / \mathrm{g}$ ).

\subsection{Tracer Infusion Profiles}

Of the eight subjects identified as outliers at the optimal exclusion level for infusion profiles (1.5 IQR), six were identified by the $\chi_{\nu}^{2}$ parameter. Five of the six subjects' TACs exhibited behavior inconsistent with a constant 30 -second infusion. Figure 4.3 shows a representative TAC of subject number 16 at stress with this abnormal behavior. 


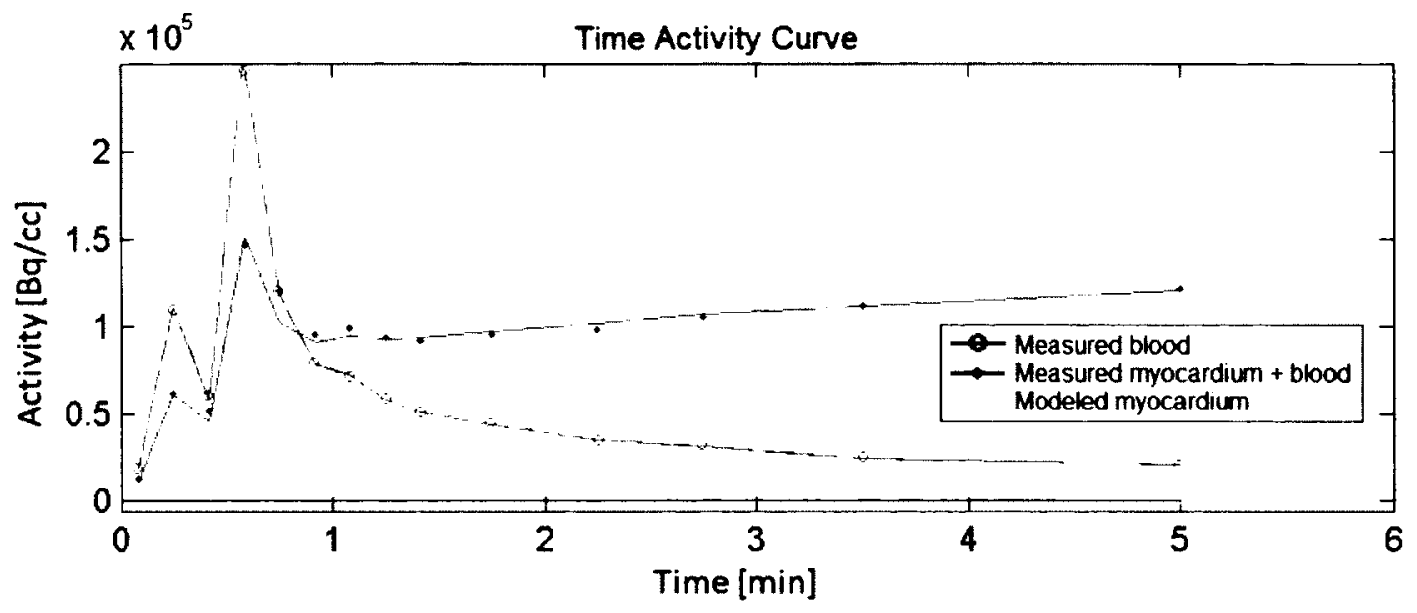

Motion Detection

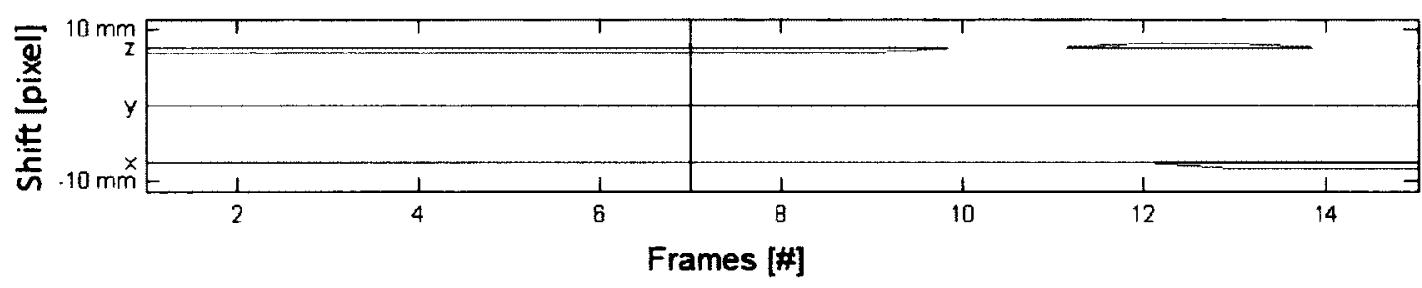

Figure 4.3: An abnormal time activity curve (top) from a subject excluded at the 1.5 IQR level of the $\chi_{v}^{2}$ goodness-of-fit parameter. The blood activity (red circles) exhibits infusion profile irregularities that are not associated with patient motion in the first four frames (bottom).

During the first-pass tracer infusion, before the recirculation of blood through the heart, the TACS should increase monotonically during the infusion phase and decrease monotonically following the peak activity. Non-monotonic patterns may coincide with gross subject motion on the scanner couch. The motion detection plot shows no evidence of a shift in the location of the myocardium during the first four frames of the scan. The issue shown in Fig. 4.3 is most likely due to a tracer infusion malfunction. This specific subject had a difference in MBF at stress of over $1.0 \mathrm{~mL} / \mathrm{min} / \mathrm{g}$, which was much 
larger than the group RPC of $0.65 \mathrm{~mL} / \mathrm{min} / \mathrm{g}$ (Table 3.9). Subject number eight was also excluded by the $T_{c}$ parameter in addition to the $\chi_{v}^{2}$. The remaining subject (number 30 ) excluded in the 1.5 IQR $\chi_{v}^{2}$ group showed a very late start in the data acquisition (> 20 seconds) which may have affected the ability of the GV fit to characterize the blood TAC compared to other scans.

The two subjects (numbers 14 and 25 ) not excluded by the $\chi_{v}^{2}$ parameter were both delta-MTT outliers at stress. Figure 4.4 shows the TACs of subject number 25 with a very long activity washout from the myocardial blood pool in the first scan that was not observed in the second. The first scan was performed to investigate the severity of a myocardial infarction (heart attack) and stenosis in the LAD. No invasive interventions were performed at the time. Slow washout of tracer from the blood pool may be due to a lower ejection fraction (pumping function) after the infarction (76). The second scan occurred three months after the first because the subject was experiencing mild chest pain. The decreased MTT in scan 2 may be due to the recovery of myocardial pumping function. However, the second subject with heterogeneous MTT scans was a normal volunteer scanned three weeks apart. The reason for this difference is unclear at this time. 

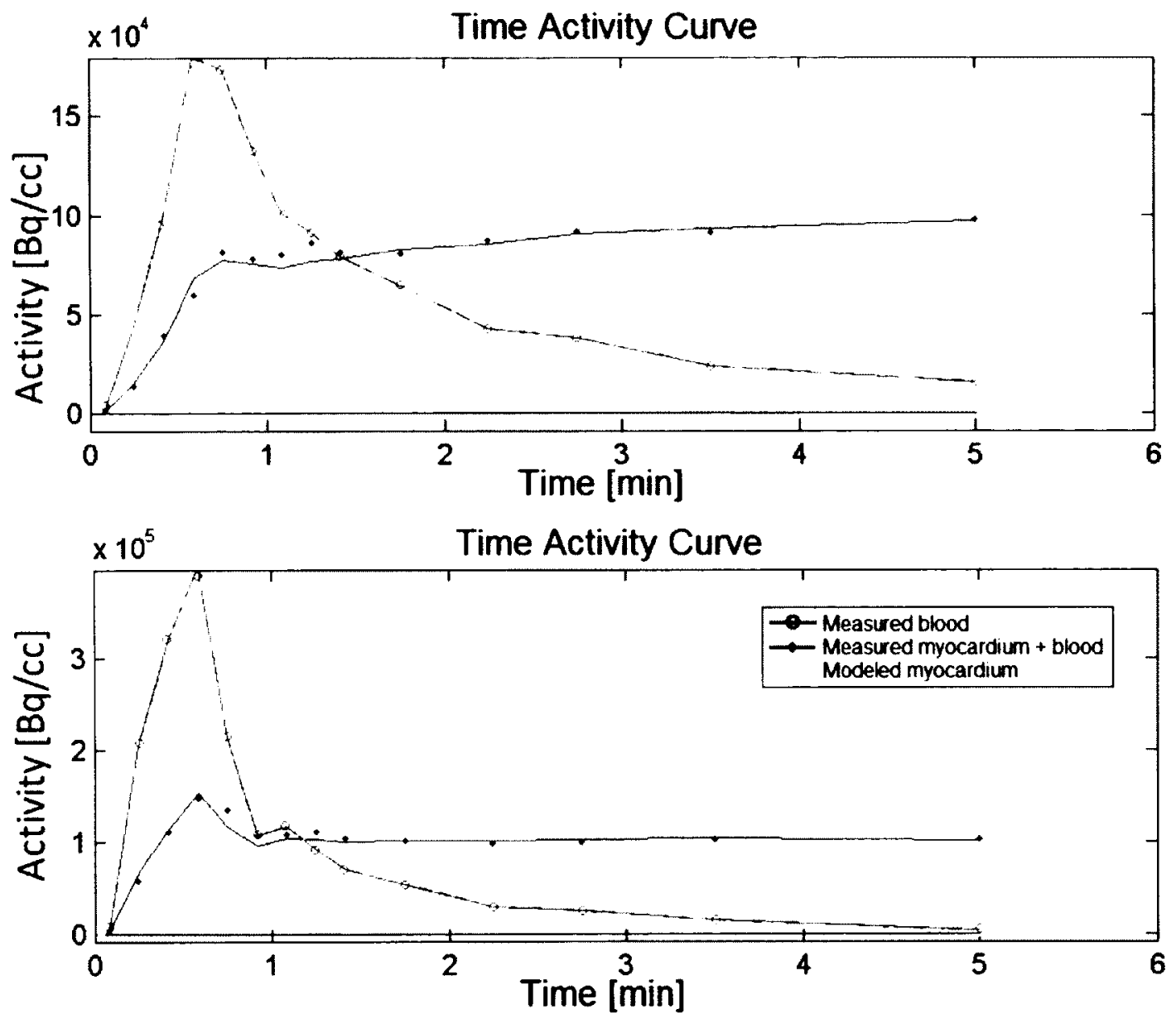

Figure 4.4: Heterogeneous time activity curves at stress from a subject excluded at the 1.5 IQR level of the mean transit-time parameter. The blood curve (red circles) of the first scan (top) shows an increased tracer clearance-time in the left atrium (70 seconds) compared to the second scan (bottom, 37 seconds) despite their similar activity rise times ( 30 seconds). MBF values were 0.63 and $0.49 \mathrm{~mL} / \mathrm{min} / \mathrm{g}$ for scan 1 and 2 respectively.

At the optimal repeatability exclusion level for profile infusions (1.5 IQR) the $T_{c}$ parameter was a redundant exclusion metric. The clearance-time outlier was also 
excluded at 2.25 IQR by the mean transit-time parameter. This subject (number eight), however, had delta-clearance-times of over 60 seconds at both rest and stress. The second scan was performed 197 days after the first to assess chest pain with no invasive procedures occurring between scans. With both scans showing the same difference, it is possible that the subject's myocardial condition changed drastically. The difference in rest $\mathrm{MBF}$ was $0.45 \mathrm{~mL} / \mathrm{min} / \mathrm{g}$ and the stress $\mathrm{MBF}$ difference was $2.61 \mathrm{~mL} / \mathrm{min} / \mathrm{g}$. The rest scans were also excluded by the delta- $\chi_{v}^{2}$ parameter at 2.25 IQR. The reason for the large differences in flow is likely a combination of all three parameters. Figures 4.5 and 4.6 show this subject's rest and stress infusion profile TACs, respectively. Overall, the three infusion profile exclusion parameters were able to distinguish five of the nine largest MBF differences at rest, five of the largest eight differences in adjusted rest, the six largest flow differences at stress, five of the top eleven in MFR, and six of the top twelve in adjusted MFR.

Very little has been published regarding the effect of infusion profile curves on repeatability. deKemp et al (58) investigated the effect of tracer infusion time on flow accuracy and precision. Scans were repeated for each infusion length but the test-retest repeatability was not reported. The potential clinical use of the serial study results may be in developing a normal database of values that would flag abnormal cases for a more thorough investigation. 

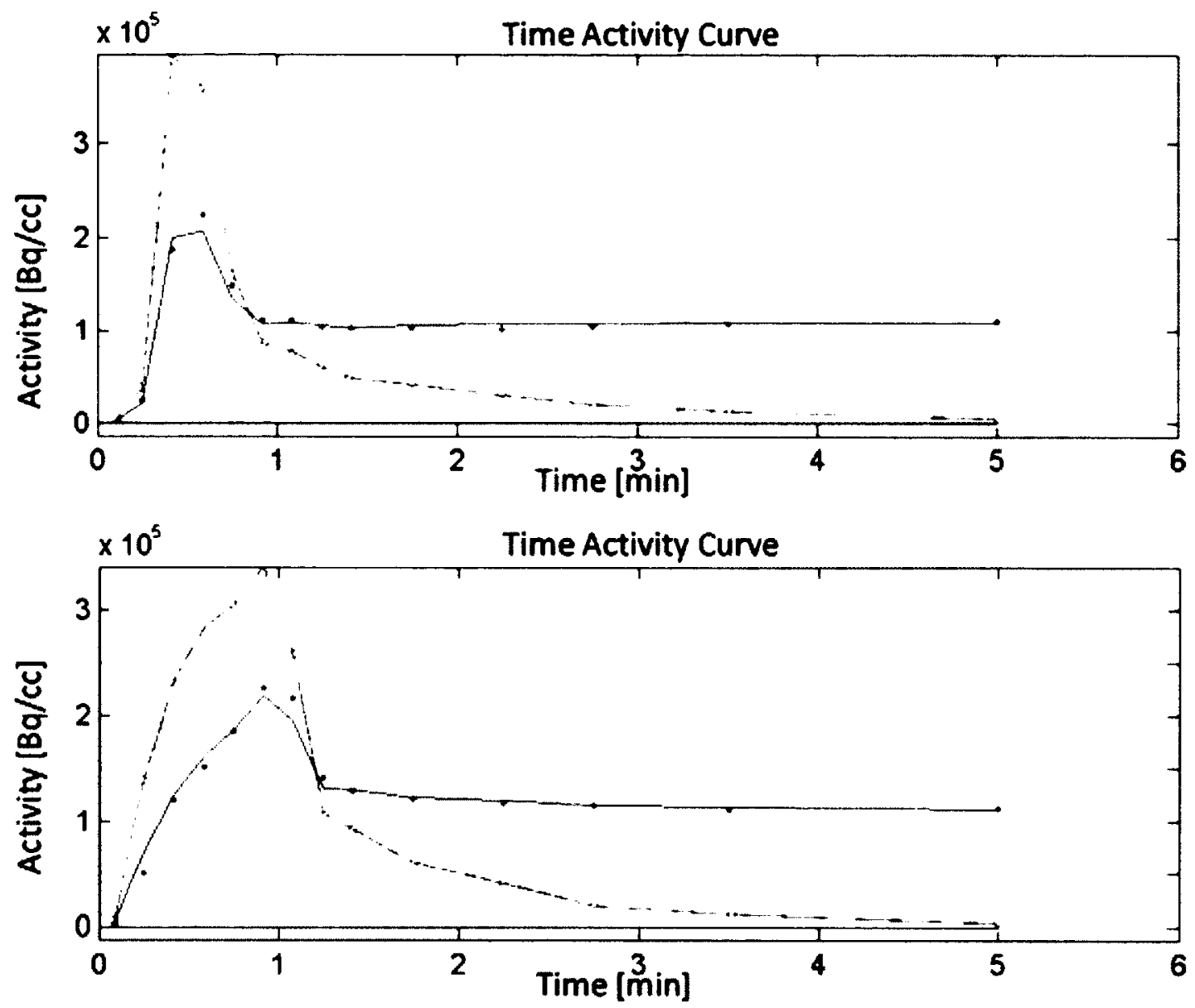

Figure 4.5: Resting time activity curves for the subject excluded at the 1.5 IQR exclusion level for the clearance-time parameter. MBFs were 1.3 and $0.82 \mathrm{~mL} / \mathrm{min} / \mathrm{g}$ for scan 1 and 2 respectively. 

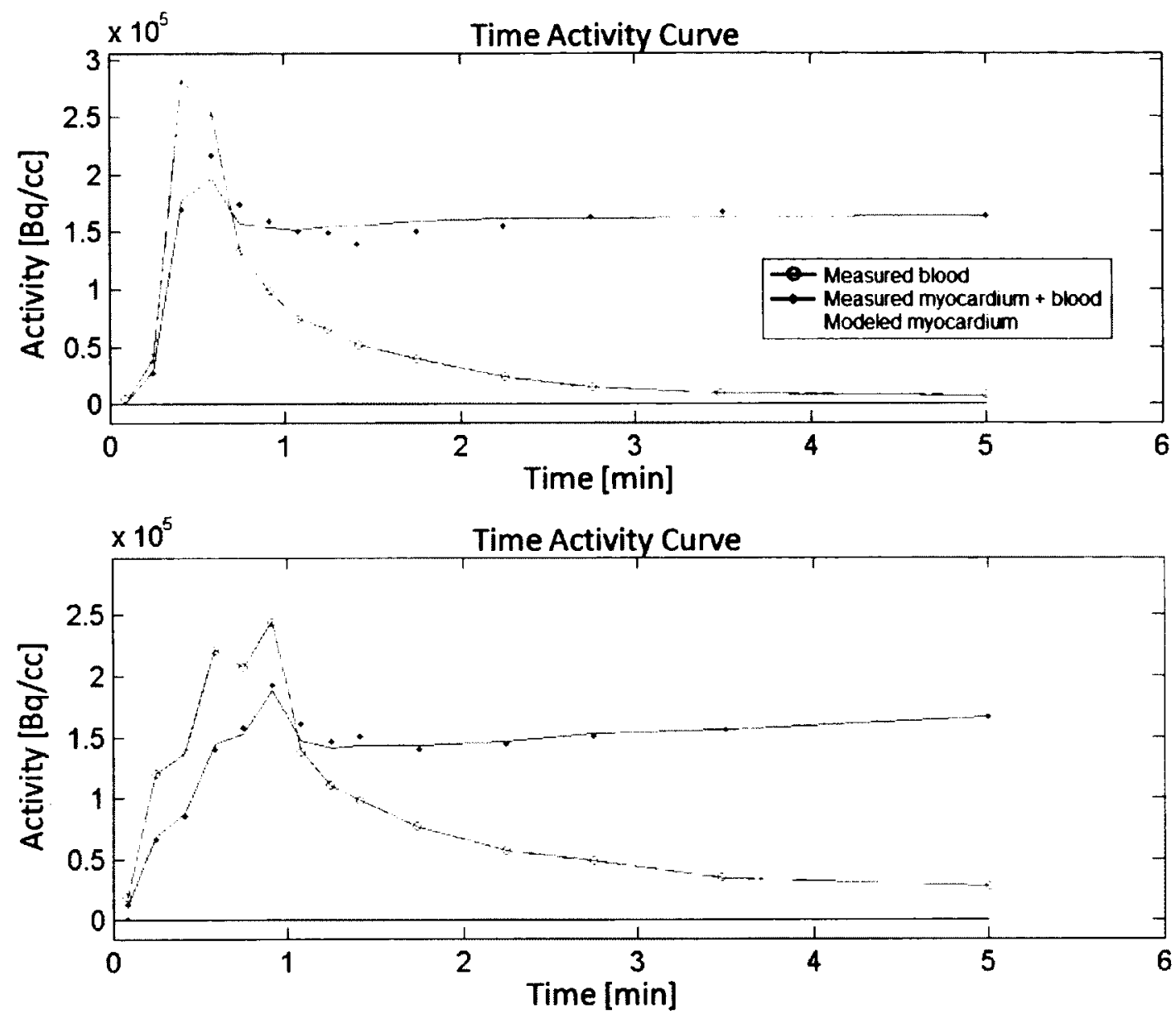

Figure 4.6: Time activity curves at stress for the same subject as in Fig. 4.5. These scans were excluded at the 1.5 IQR exclusion level for the clearance-time parameter. MBFs were 4.5 and $1.8 \mathrm{~mL} / \mathrm{min} / \mathrm{g}$ for scan 1 and 2 respectively.

A comparison of RPP values between the same-day and serial studies at rest showed a significant difference. Same-day RPP values were significantly more repeatable ( $p<$ 0.001). It is likely that the significant increase in time between scans, as opposed to 


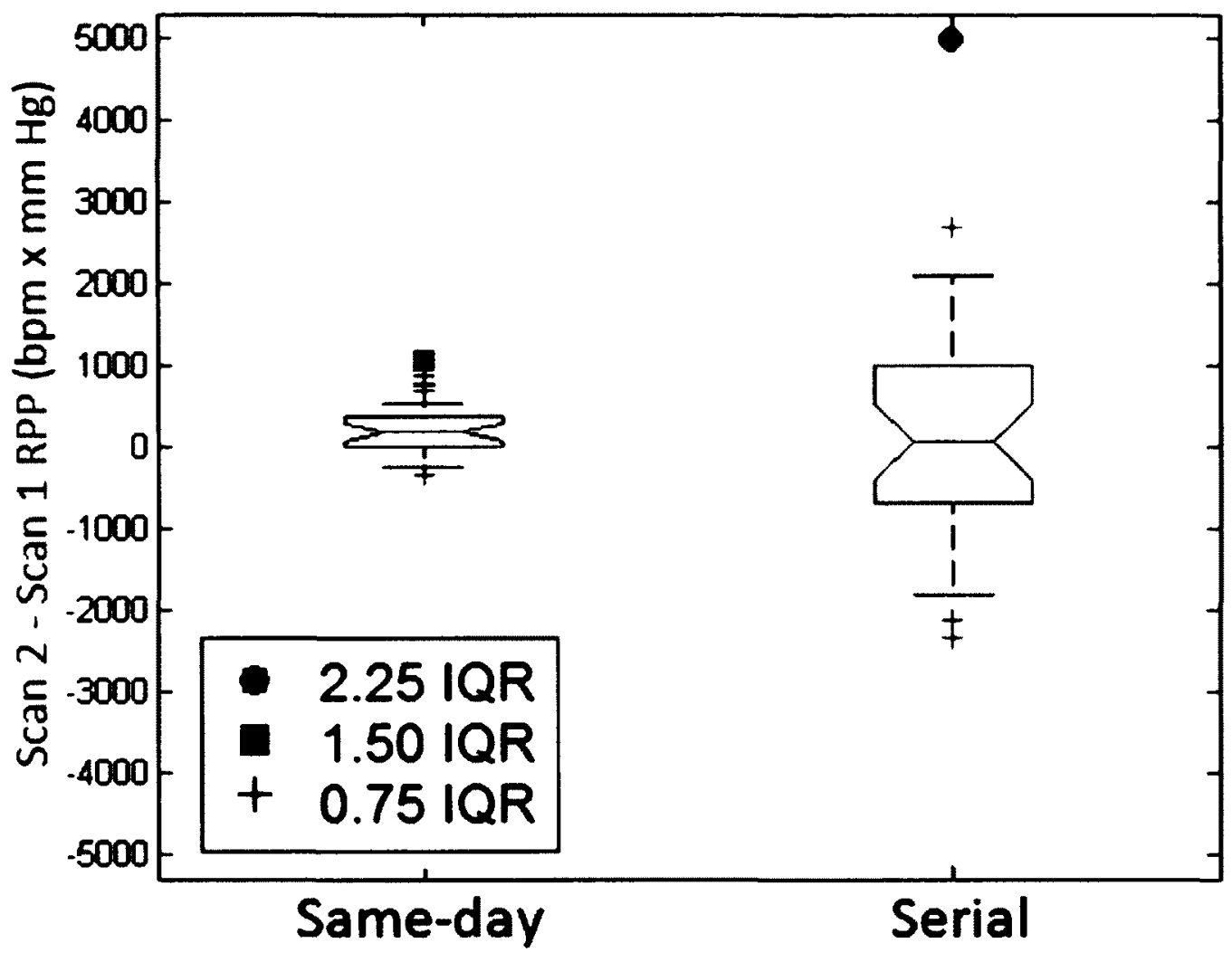

Figure 4.7: Distribution of rate-pressure-products box plot comparison between same-day and serial study groups. Outliers at each exclusion level are noted with a unique symbol.

changes in subject disease status, are the reason for the difference. Figure 4.7 shows box plots of the distribution of values.

One subject was excluded at 1.5 IQR and six subjects at 0.75 IQR. Rest MBF and RPP adjusted MBF for both same-day 1.5 and 0.75 IQR groups were not significantly different vs. the whole population or each other, for either scan 1 or 2 ( $p=N S$ ). None of the subjects in the same-day study would have been excluded by any of the serial study 
RPP exclusion parameters; the largest same-day delta-RPP was smaller than the 0.75 IQR inclusion limit in the serial study.

\subsection{Test-Retest Repeatability}

Table 4.1 shows the results of the present studies using the OSEM-6-LA-SOC protocol in comparison to previous papers that have reported rest MBF test-retest repeatability. Manabe et al (57), Kaufmann et al (55), and Wyss et al (56) all reported RPC values 10.19 $\mathrm{mL} / \mathrm{min} / \mathrm{g}$, which was $24 \%$ of the mean MBF, 0.17 which was $19 \%$ of the mean, and 0.26 , which was $21 \%$ of the mean, respectively) in same-day, young normal subjects, similar to present study results measured both in normal subjects and in CAD patients. Schindler et al (51) found consistent repeatability between same-day $(0.26,39 \%)$ and two- to three-week intervals $(0.26,40 \%)$ in both healthy volunteers and in CAD patients using cold-pressor testing. Jagathesan et al $(54)$ reported the most repeatable MBF in a long-term (24 week interval) study $(0.25,24 \%)$ in older CAD patients. El Fakhri et al (30) calculated a combined coefficient of reproducibility of $0.45 \mathrm{~mL} / \mathrm{min} / \mathrm{g}$ for rest and stress data, also similar to the present serial study findings. Same-day protocols were generally more repeatable than studies done over a number of weeks. However, none of the studies reported the effects of RPP adjustment in relation to MBF repeatability. The repeatability of resting ${ }^{82} \mathrm{Rb} \mathrm{MBF}$ in the same-day study using the OSEM-6-LA-SOC protocol is comparable to what has been reported in the literature. 
Table 4.1: Test-retest repeatability of rest MBF compared to published literature

\begin{tabular}{|c|c|c|c|c|c|c|c|}
\hline Study & Tracer & Rest MBF & RPC & $\%$ & $\begin{array}{c}\text { Rest MBF } \\
\text { RPP }\end{array}$ & $\begin{array}{l}\text { RPC } \\
\text { RPP }\end{array}$ & $\%$ \\
\hline Same-day ${ }^{a}$ & ${ }^{82} R b$ & $0.84 \pm 0.44$ & 0.21 & 25 & $0.86 \pm 0.31$ & 0.20 & 23 \\
\hline Serial $1.51 Q R^{b}$ & ${ }^{82} R b$ & $0.66 \pm 0.23$ & 0.26 & 40 & $0.67 \pm 0.21$ & 0.21 & 32 \\
\hline Serial $0.751 Q R^{c}$ & ${ }^{82} R b$ & $0.66 \pm 0.23$ & 0.27 & 41 & $0.68 \pm 0.23$ & 0.19 & 28 \\
\hline Manabe (57) & ${ }^{82} \mathrm{Rb}$ & $0.77 \pm 0.25$ & 0.19 & 24 & $0.99 \pm 0.29$ & 0.25 & 25 \\
\hline Sdringola (69) & ${ }^{82} \mathrm{Rb}$ & $0.72 \pm 0.17$ & 0.25 & 35 & $\mathrm{n} / \mathrm{a}$ & $n / a$ & $n / a$ \\
\hline El Fakhri (30) & ${ }^{82} \mathrm{Rb}$ & $1.13 \pm 0.19$ & $0.45^{d}$ & $\mathrm{n} / \mathrm{a}$ & $1.18 \pm 0.32$ & $\mathrm{n} / \mathrm{a}$ & $\mathrm{n} / \mathrm{a}$ \\
\hline Wyss (56) & $\mathrm{H}_{2}{ }^{15} \mathrm{O}$ & $1.22 \pm 0.16$ & 0.26 & 21 & $1.75 \pm 0.37$ & 0.58 & 37 \\
\hline Kaufmann (55) & $\mathrm{H}_{2}{ }^{15} \mathrm{O}$ & $0.89 \pm 0.14$ & 0.17 & 19 & $1.25 \pm 0.22$ & 0.28 & 22 \\
\hline Jagathesan (54) & $\mathrm{H}_{2}{ }^{15} \mathrm{O}$ & $1.03 \pm 0.19$ & 0.25 & 24 & $\mathrm{n} / \mathrm{a}$ & $\mathrm{n} / \mathrm{a}$ & $n / a$ \\
\hline Schindler $(51)^{e}$ & ${ }^{13} \mathrm{NH}_{3}$ & $0.67 \pm 0.19$ & 0.26 & 39 & $\mathrm{n} / \mathrm{a}$ & $\mathrm{n} / \mathrm{a}$ & $\mathrm{n} / \mathrm{a}$ \\
\hline Schindler $(51)^{f}$ & ${ }^{13} \mathrm{NH}_{3}$ & $0.65 \pm 0.16$ & 0.26 & 40 & $\mathrm{n} / \mathrm{a}$ & $\mathrm{n} / \mathrm{a}$ & $n / a$ \\
\hline Nagamachi (52) & ${ }^{13} \mathrm{NH}_{3}$ & $0.62 \pm 0.14$ & $0.25^{8}$ & 39 & $0.62 \pm 0.07$ & $0.17^{\mathrm{g}}$ & 27 \\
\hline \multicolumn{8}{|c|}{$\begin{array}{l}\text { a First study using LA input function, OSEM reconstruction, } 6 \text { minute scan time an } \\
\text { spillover correction. } \\
{ }^{b} \text { Second study using } 1.5 \text { IQR infusion profile and hemodynamic inclusion groups } \\
\text { c Second study using } 1.5 \text { IQR infusion profile } \& 0.75 \text { IQR hemodynamic inclusion g } \\
{ }^{d} \text { Combined rest and stress, }{ }^{\text {e }} \text { Same-day scan interval, }{ }^{f} 2-3 \text { week scan interval, } \\
{ }^{B} \text { Estimated from reported results } \\
\text { Myocardial blood flow (MBF) and repeatability coefficient (RPC) are in } \mathrm{mL} / \mathrm{min} / \mathrm{g} \\
\text { RPP =rate-pressure-product adjusted, } \% \text { is the RPC / mean MBF } \times 100 \%\end{array}$} \\
\hline
\end{tabular}

The RPCs of same-day rest MBF, with the RPP analysis shown in Figure 4.7, were not significantly affected by excluding RPP outliers. Repeatability of the same-day RPP adjusted rest MBF values were similar to the sub-group of serial study subjects who had repeatable infusion profiles and rest-stress hemodynamics.

Table 4.2 shows the stress MBF results of the present studies compared to published literature. Studies with same-day scan protocols reported more repeatable stress MBF 
Table 4.2: Test-retest repeatability of stress MBF compared to published literature

\begin{tabular}{|c|c|c|c|c|c|c|c|}
\hline Study & Tracer & Pop. & Recon & $\begin{array}{l}\text { Stress } \\
\text { Agent }\end{array}$ & Stress MBF & RPC & $\%$ \\
\hline Same-day ${ }^{a}$ & ${ }^{82} R b$ & Mixed & OSEM & Dipyridamole & $1.94 \pm 1.12$ & $n / a$ & $n / a$ \\
\hline Serial 1.5 IQR ${ }^{b}$ & ${ }^{82} R b$ & Mixed & OSEM & Dipyridamole & $1.66 \pm 0.60$ & 0.65 & 40 \\
\hline Serial $0.751 Q R^{c}$ & ${ }^{82} R b$ & Mixed & OSEM & Dipyridamole & $1.68 \pm 0.65$ & 0.53 & 32 \\
\hline Manabe (57) & ${ }^{82} \mathrm{Rb}$ & Normal & FBP & ATP & $3.35 \pm 1.37$ & 0.92 & 27 \\
\hline Sdringola (69) & ${ }^{82} \mathrm{Rb}$ & Normal & FBP & Dipyridamole & $2.90 \pm 0.50$ & 0.99 & 34 \\
\hline El Fakhri (30) & ${ }^{82} \mathrm{Rb}$ & Mixed & OSEM & Dipyridamole & $2.81 \pm 1.02$ & $0.45^{d}$ & $\mathrm{n} / \mathrm{a}$ \\
\hline Wyss (56) & $\mathrm{H}_{2}{ }^{15} \mathrm{O}$ & Normal & FBP & Adenosine & $5.13 \pm 0.74$ & 1.34 & 27 \\
\hline Kaufmann (55) & $\mathrm{H}_{2}{ }^{15} \mathrm{O}$ & Normal & FBP & Adenosine & $3.51 \pm 0.45$ & 0.90 & 25 \\
\hline Jagathesan (54) & $\mathrm{H}_{2}{ }^{15} \mathrm{O}$ & CAD & FBP & Dobutamine & $2.02 \pm 0.44$ & 0.58 & 28 \\
\hline Schindler $(51)^{\mathrm{e}}$ & ${ }^{13} \mathrm{NH}_{3}$ & Mixed & FBP & Cold Pressor & $0.88 \pm 0.21$ & 0.28 & 32 \\
\hline Schindler $(51)^{f}$ & ${ }^{13} \mathrm{NH}_{3}$ & Mixed & FBP & Cold Pressor & $0.85 \pm 0.19$ & 0.31 & 37 \\
\hline Nagamachi (52) & ${ }^{13} \mathrm{NH}_{3}$ & Normal & FBP & Adenosine & $2.00 \pm 0.39$ & $0.56^{\mathrm{g}}$ & 28 \\
\hline \multicolumn{8}{|c|}{$\begin{array}{l}{ }^{a} \text { First study using LA input function, OSEM reconstruction, } 6 \text { minute scan time and dual } \\
\text { spillover correction. ATP = Adensine triphosphate } \\
\text { b Second study using } 1.5 \text { IQR infusion profile and hemodynamic inclusion groups } \\
{ }^{c} \text { Second study using } 1.5 \text { IQR infusion profile } \& 0.75 \text { IQR hemodynamic inclusion groups } \\
{ }^{d} \text { Combined rest and stress, e Same-day scan interval, }{ }^{f} 2-3 \text { week scan interval } \\
\text { g Estimated from reported results }\end{array}$} \\
\hline
\end{tabular}

values (51) (55) (56) (57) than long-term studies (51) (52) (54) (59). The increase in repeatability from same-day to long-term studies (a few weeks to two months) is thought to be caused by differences in RPP rather than changes in disease status. Removing subjects with both different tracer infusion profiles and different RPPs both significantly lowered the repeatability at stress in the serial study.

Optimizing the repeatability of resting MBF should also have important effects on stress/rest MFR. While the same-day study was unable to incorporate a second stress 
scan with the standard administration of aminophylline (vasodilatation antidote), the single stress scan was paired with both rest scans to simulate single-session MFR measurements. Neglecting the added variability that might be expected from repeated stress scans, the RPC values measured in the present study (22-24\%) are much smaller than what has been reported previously in the literature as shown in Table 4.3. The variability associated with repeated stress scans is a significant factor, suggesting that the present study results may be interpreted as a lower bound on MFR repeatability due to subject and imaging variability. Overall, large differences in RPP $(>2,000 \mathrm{bpm} \times \mathrm{mm}$ $\mathrm{Hg})$

Table 4.3: Test-retest repeatability of myocardial flow reserve (MFR) compared to published literature

\begin{tabular}{|c|c|c|c|c|c|c|c|}
\hline Study & Tracer & MFR & RPC & $\%$ & MFR RPP & RPC RPP & $\%$ \\
\hline Same-day ${ }^{\circ}$ & ${ }^{82} R b$ & $2.38 \pm 0.96$ & 0.58 & 24 & $2.32 \pm 0.83$ & 0.52 & 22 \\
\hline Serial $1.51 Q R^{b}$ & ${ }^{82} R b$ & $2.66 \pm 0.86$ & 1.30 & 48 & $2.50 \pm 0.65$ & 1.10 & 45 \\
\hline Serial $0.75 \mathcal{I Q R}^{c}$ & ${ }^{82} R b$ & $2.64 \pm 0.82$ & 0.78 & 29 & $2.50 \pm 0.66$ & 0.95 & 37 \\
\hline Manabe (57) & ${ }^{82} \mathrm{Rb}$ & $4.47 \pm 1.47$ & 1.61 & 36 & $3.46 \pm 1.22$ & 1.20 & 34 \\
\hline Sdringola (69) & ${ }^{82} \mathrm{Rb}$ & $4.03 \pm 0.84$ & 1.53 & 38 & $\mathrm{n} / \mathrm{a}$ & $n / a$ & $n / a$ \\
\hline Wyss (56) & $\mathrm{H}_{2}{ }^{15} \mathrm{O}$ & $4.23 \pm 0.62$ & 1.44 & 35 & $3.06 \pm 0.84$ & 1.48 & 44 \\
\hline Kaufmann (55) & $\mathrm{H}_{2}{ }^{15} \mathrm{O}$ & $4.05 \pm 0.75$ & 1.32 & 33 & $2.90 \pm 0.70$ & 0.98 & 34 \\
\hline Jagathesan (54) & $\mathrm{H}_{2}{ }^{15} \mathrm{O}$ & $1.98 \pm 0.40$ & 0.64 & 33 & $\mathrm{n} / \mathrm{a}$ & $n / a$ & $n / a$ \\
\hline
\end{tabular}

\footnotetext{
${ }^{a}$ First study using a single stress scan with repeated rest scans to evaluate MFR

${ }^{b}$ Second study using 1.5 IQR infusion profile and hemodynamic inclusion groups

${ }^{c}$ Second study using 1.5 IQR infusion profile \& 0.75 IQR hemodynamic inclusion groups Myocardial flow reserve (MFR) is unit-less ratio of stress/rest MBF, repeatability coefficient $(\mathrm{RPC})$ is in $\mathrm{mL} / \mathrm{min} / \mathrm{g}, \mathrm{RPP}=$ rate-pressure-product adjusted, \% is the RPC / mean MFR $\times 100 \%$
} 
from scan to scan were associated with less repeatable rest MBF and MFR values and significantly less repeatable stress MBF values $(p<0.03)$.

Klein et al (50) determined that the intra-operator variability (CV) for quantification of absolute MBF using the FlowQuant program was approximately 6\%. Optimized testretest repeatability in the same-day study was $12 \%$ using the OSEM-6-LA-SOC-RPP protocol. Since all test and retest studies were processed by the same operator in this study, approximately half of the total variability may be attributed to operator interactions (required in $\sim 10 \%$ of cases), and the remaining half attributed to the true test-retest differences in measured MBF values. Lamoureux et al (77) showed that rest MBF repeatability in rat studies was influenced minimally by operator intervention.

Optimizing the repeatability of the same-day resting protocol had a significant effect on the repeatability of MFR. Figure 4.8 shows the repeatability of the same-day in comparison with serial repeatability. Flow reserve has been shown to be a predictor of adverse cardiac outcomes (78). If the repeatability of flow reserve is minimized through careful measurement and optimized protocols at both rest and stress, then more accurate risk stratification and appropriate decisions can be made regarding patient health and management of CAD. 
The test-retest repeatability results presented in this study may not be exactly the same as those obtained using other flow quantification programs. However, it was demonstrated recently (79) that very similar $\operatorname{MBF}(\Delta<4-12 \%)$ and flow reserve $(\Delta<8 \%)$ values are obtained using FlowQuant compared to two commercial software implementations of the same one-tissue-compartment model of rubidium kinetics (64), suggesting that the same physiologic and gender effects may be generalizable to MBF measurements made using the same or similar software programs. 


\section{Chapter 5: Conclusions}

Many factors affect the accurate measurement of quantitative MBF, more still for optimizing the test-retest repeatability. A protocol for achieving optimal test-retest repeatability in same-day ${ }^{82} \mathrm{Rb}$ PET scans was investigated under resting conditions, and the effects of many of the factors were studied. The resulting protocol with the best repeatability at rest was used subsequently to determine the test-retest repeatability at rest and stress in a group consisting of mostly CAD patients and the effects of two littlestudied factors were investigated: tracer infusion profile and intra-subject hemodynamic variability.

Same-day resting blood flow was measured with good repeatability in the LV myocardium using ${ }^{82} \mathrm{Rb}$ PET; $95 \%$ of same-day test-retest differences were within 0.20 $\mathrm{mL} / \mathrm{min} / \mathrm{g}$, which was $25 \%$ of the average rest MBF value. The optimal imaging protocol used OSEM iterative reconstruction, a six-minute scan time, an image-derived input function using a blood region located in the left atrium cavity, dual-spillover correction, and rate-pressure-product adjustment for differences in subject hemodynamics. There was no significant difference in repeatability between CAD patients and normal volunteers even though the RPC was $50 \%$ larger in CAD patients $(p=0.3)$. A larger population might confirm this difference in repeatability but it seems more likely that there is no RPC difference between normal volunteers and patients with stable CAD. Serial measurement of test-retest repeatability on different days produced a similar RPC 
of $0.19 \mathrm{~mL} / \mathrm{min} / \mathrm{g}$, which was $28 \%$ of the average rest MBF value, after subjects with heterogeneous hemodynamic conditions and scans with heterogeneous infusion profile curves were excluded. The values from both studies agreed with published literature.

In the same-day study, a single stress scan was used to evaluate the effect of resting flow values on MFR repeatability, removing a significant source of physiologic variability that may be expected from repeated stress measurements. The repeatability of serial stress MBF was measured with the protocol optimized for resting MBF and may not be the best to use for stress scans. However, when subjects with heterogeneous scan parameters, as described above, were removed from the study, the remaining subgroup had repeatability similar to published values. Repeatability of stress MBF measurements will be influenced by heterogeneity of the physiologic response to dipyridamole stress and amplified by decreased tracer extraction at higher flows. Differences in rate-pressure-products and infusion profiles had a much larger impact on stress flow than rest, suggesting that achieving similar vasodilation response to pharmaceuticals and identical infusion methods are critical in measuring the repeatability of stress MBF accurately. These effects should be investigated further to optimize the measurement of stress MBF and stress/rest flow reserve with ${ }^{82}$ Rb PET.

The best measure of MFR will be the result of optimized rest and stress scans. The repeatability results of serial MFR with hemodynamic and infusion profile sub-groups were comparable to published literature. However, the RPC is still relatively large to 
accurately determine absolute limits or make definitive diagnoses based on individual patient MFR values when compared to clinical reports on flow reserve and adverse outcomes (78). Lowering the RPC for MFR is therefore necessary, and future research should be directed towards this goal.

The improved image quality of OSEM reconstruction was able to provide more repeatable MBF results. This was expected as many studies had previously shown that improved iterative methods were superior to FBP. The underestimation of MBF by OSEM compared to FBP was only seen when spillover correction was applied. Although the correction was instrumental in achieving the optimal repeatability, the use of an LA blood input function may mitigate the effect of MBF underestimation by the SOC. If spillover from the myocardial walls is no longer a factor associated with the location of the input function, it may be much better to provide an elegant solution (LA input) than attempt to correct a poor one (LV wall motion and contamination of the blood pool). One shortcoming of this research is that all of the possible optimizing combinations were not investigated that could have provided answers to the issue above.

The benefits of shortening the scan length were shown in the same-day rest study. Reducing the possibility of subject motion, improving patient comfort and scanning experience, and the possibility of more patient throughput are superb compensation for the minute gain in counting statistics gained in the final four-minute time frame. With 
the fixed cost of the ${ }^{82} \mathrm{Rb}$ generator, each extra patient scanned reduces the cost per dose (80).

As a result of the serial rest-stress repeatability study, important clinical changes were made to imaging acquisition procedures to ensure that the initial dynamic PET data would be recorded. Even with the constant-activity infusion system there were a number of scans $(\sim 5 \%)$ that showed inconsistent blood input functions, and these had a detrimental effect on the repeatability of MBF quantification. Quality assurance of the infusion system is essential in delivering the proper dose as a single bolus, or constant infusion, so that accurate blood-to-myocardium modeling is achieved.

This research set out to determine a the best protocol for rest MBF regarding test-retest repeatability, the repeatability of MBF and MFR for serially imaged subjects, and the effect of heterogeneous infusion profiles and hemodynamics on test-retest repeatability. This work contributes to the knowledge of technical and clinical factors that affect test-retest repeatability at the $\mathrm{UOHI}$ and at other diagnostic centers that employ similar imaging and analysis protocols.

\subsection{Future Work}

Future areas of research include studying the effect of physiologic heart size on MBF and possible bias in measuring blood volumes as shown in the same-day study. To our knowledge this effect has not been reported previously in CAD patients, and may 
warrant further investigation. A study designed to systematically alter infusion profiles, and confirm the effects on test-retest repeatability of MBF measurements in same-day scans, would be a sound extension of the current work. Obtaining same-day stress scans to complete the protocol optimization, and confirm the best protocol for measuring the test-retest repeatability of MFR, would significantly add to the results found in this thesis.

In some subjects the pharmacological vasodilator may not have the desired maximal vasodilatation effect, e.g., in cases where subjects have consumed caffeine. It is also unknown to what extent differing responses to stress agents from scan to scan will affect test-retest repeatability. Investigating the effect that differences in the increased hemodynamic response of stress over rest may have on MBF repeatability could add to understanding the full effects of hemodynamics on quantitative MBF and test-retest repeatability. The results presented in this thesis help to improve understanding of the test-retest repeatability of ${ }^{82} \mathrm{Rb}$ PET and the factors that affect MBF and MFR measurements. This research has the potential to aid in the assessment and clinical interpretation of MBF over time with serial, or longitudinal, studies in patients and in animal models of disease and therapy. 


\section{Appendix A}

\section{Repeatability Coefficient (RPC)}

Bland and Altman introduced a graphical method to compare two measurement techniques (63).The difference between the measurements is plotted versus the mean of the measurements. The RPC is calculated as $1.96 \times$ the standard deviation of the differences and is a measure of the variation between the measurement techniques. The significance of 1.96 is that $95 \%$ of repeated measurements between the two techniques will be less than the RPC. In treating and diagnosing patients who have had repeated quantitative MBF analysis, it is necessary to know if a change in MBF is within the accepted variability. Patients whose repeated MBF values are greater than the RPC are likely to have experienced a progression, for better or worse, in disease status. 


\section{Appendix B}

\section{Box-Plot Analysis}

A box-plot is a non-parametric display of data distributions (81). This method of analysis was chosen for this research because a few study subjects had outlier values that were large compared to the mean population values, and the box-plot is robust against the effects of outliers. Outliers have the effect of skewing the measurement of mean and standard deviation and misrepresenting the sample distribution when the data are not normally distributed. Figure B.1 shows a box-plot of MBF values with the important statistics identified. The horizontal line in the center of the box is the sample median. The top and bottom of the box are the first and third, or upper and lower, quartiles of the data. This means that $25 \%$ of the data points are below the lower quartile, $25 \%$ of the data points are between the lower quartile and the median, and so on. The middle $50 \%$ of the data, between the upper and lower quartiles, is the interquartile range (IQR). The whisker above the box extends to the last data point that is less than the upper quartile value plus $1.5 \times$ IQR ( 1.5 is the standard box-plot whisker limit as it corresponds to two standard deviations for normally distributed data). Any data point, marked with a ' + ' symbol, that exceeds this limit is considered an 'outlier'. The angled sides of the box are called notches and represent $95 \%$ confidence intervals on the median. If the notches of two box-plots overlap, then their medians are not statistically different. 


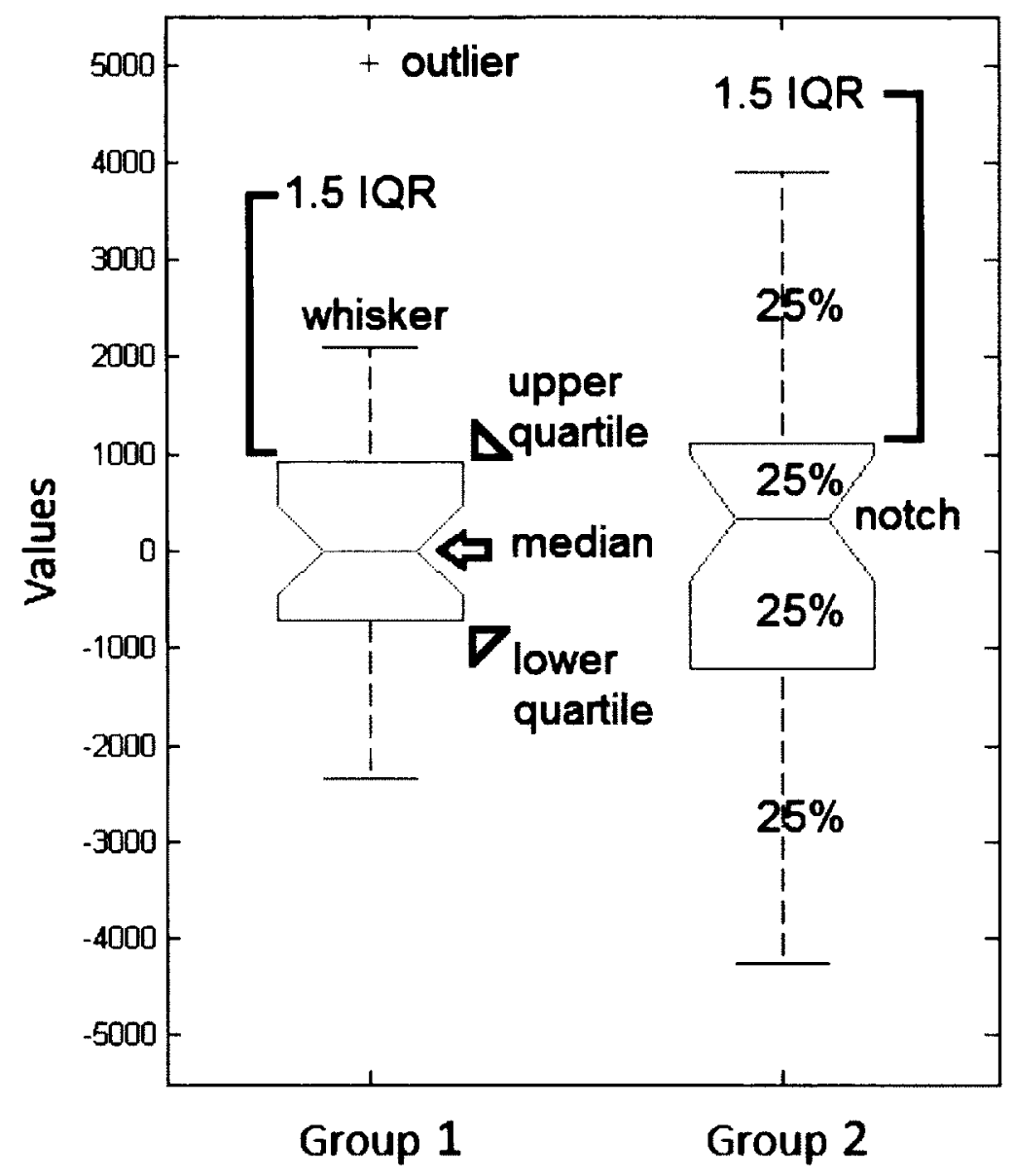

Figure B.1: A notched box-plot comparison between two groups. 


\section{Appendix C: Extended Data}

\section{Same Day Subjects}

\section{Population Statistics}

$\begin{array}{ccccrrr}\text { Subject } & \text { Gender } & \text { Age } & \text { Status } & \text { BMI } & \text { RPP1 } & \text { RPP2 } \\ 1 & \text { f } & 47 & & 23.9 & 5879 & 5829 \\ 2 & \text { m } & 42 & & 32.8 & 5891 & 6934 \\ 3 & \text { m } & 72 & \text { CAD } & 24.9 & 6235 & 6210 \\ 4 & \text { m } & 67 & \text { CAD } & 41.8 & 7696 & 8473 \\ 5 & \text { f } & 74 & \text { CAD } & 31.8 & 9203 & 9942 \\ 6 & \text { f } & 67 & \text { CAD } & 27.5 & 12667 & 12863 \\ 7 & \text { m } & 39 & & 27.7 & 5093 & 5205 \\ 8 & \text { f } & 68 & \text { CAD } & 33.9 & 7289 & 8030 \\ 9 & \text { f } & 60 & & 30.9 & 7704 & 7740 \\ 10 & \text { f } & 31 & & 21 & 5869 & 6269 \\ 11 & \text { m } & 51 & \text { CAD } & 30.4 & 6077 & 5966 \\ 12 & \text { m } & 68 & \text { CAD } & 31.3 & 6840 & 7719 \\ 13 & \text { f } & 58 & \text { CAD } & 41.5 & 9834 & 10074 \\ 14 & \text { f } & 65 & \text { CAD } & 27.9 & 8631 & 8941 \\ 15 & \text { m } & 70 & \text { CAD } & 29.8 & 6567 & 6325 \\ 16 & \text { f } & 48 & \text { CAD } & 52.9 & 11461 & 11448 \\ 17 & \text { m } & 46 & \text { CAD } & 39.9 & 4875 & 4922 \\ 18 & \text { m } & 56 & \text { CAD } & 36.3 & 9028 & 8888 \\ 19 & \text { m } & 27 & & 23.7 & 5562 & 5775 \\ 20 & \text { m } & 57 & \text { CAD } & 34.9 & 6682 & 6831 \\ 21 & \text { f } & 48 & \text { CAD } & 34.2 & 7952 & 8117 \\ 22 & \text { f } & 53 & \text { CAD } & 39.2 & 6832 & 6949 \\ 23 & \text { m } & 62 & \text { CAD } & 27.5 & 5154 & 5341 \\ 24 & \text { m } & 64 & \text { CAD } & 19.9 & 4600 & 4873 \\ 25 & \text { f } & 59 & \text { CAD } & 39.9 & 8738 & 8552 \\ 26 & \text { m } & 64 & \text { CAD } & 23.7 & 5607 & 6128 \\ 27 & \text { m } & 38 & & 29.3 & 5883 & 5528 \\ 28 & \text { m } & 61 & \text { CAD } & 31.8 & 5488 & 5830 \\ 29 & \text { m } & 65 & \text { CAD } & 28.7 & 6375 & 7293 \\ 30 & \text { m } & 43 & & 25.2 & 4818 & 4884 \\ 31 & \text { m } & 39 & & 25.1 & 9338 & 9321 \\ 32 & \text { m } & 76 & \text { CAD } & 31.9 & 9240 & 6900 \\ 33 & \text { m } & 45 & \text { CAD } & 33.7 & 6150 & 6355 \\ 34 & \text { m } & 53 & \text { CAD } & 27.7 & 6030 & 6180 \\ 35 & \text { m } & 62 & \text { CAD } & 35.3 & 7541 & 8236 \\ 36 & \text { m } & 53 & \text { CAD } & 33.7 & 14947 & 16019\end{array}$

061 = OSEM-6 min-scan 1, soc = spillover corrected 


\section{Left Ventricle ROI Arterial Input Function - Rest and Stress MBF}

$\begin{array}{ccccccc}\text { Subject } & \text { Rest } & \text { Rest } & \text { Rest } & \text { Rest } & \text { Stress } & \text { Stress } \\ & 061 & \text { o6 soc 1 } & 062 & \text { o6 soc 2 } & \text { o6 } & \text { o6 soc } \\ 1 & 0.70 & 0.59 & 0.62 & 0.64 & 2.77 & 2.61 \\ 2 & 0.70 & 0.56 & 0.83 & 0.60 & 2.78 & 2.74 \\ 3 & 0.71 & 0.53 & 0.68 & 0.50 & 1.78 & 1.57 \\ 4 & 0.88 & 0.77 & 1.10 & 0.96 & 2.30 & 2.12 \\ 5 & 1.47 & 1.17 & 1.20 & 1.00 & 1.81 & 1.53 \\ 6 & 1.62 & 1.44 & 2.26 & 1.66 & 3.87 & 3.26 \\ 7 & 0.65 & 0.61 & 0.62 & 0.51 & & \\ 8 & 0.73 & 0.74 & 1.03 & 1.04 & 2.30 & 2.20 \\ 9 & 0.81 & 0.73 & 0.93 & 0.91 & & \\ 10 & 0.96 & 0.95 & 1.03 & 0.90 & 3.62 & 3.68 \\ 11 & 0.77 & 0.55 & 0.63 & 0.46 & 1.47 & 1.11 \\ 12 & 0.90 & 0.88 & 0.95 & 0.78 & 0.64 & 0.69 \\ 13 & 1.10 & 0.93 & 0.92 & 0.77 & 1.80 & 1.85 \\ 14 & 1.40 & 1.21 & 1.01 & 0.88 & 3.83 & 3.16 \\ 15 & 1.20 & 1.14 & 1.18 & 1.30 & 1.97 & 1.74 \\ 16 & 1.02 & 0.94 & 0.73 & 0.68 & 1.38 & 1.23 \\ 17 & 0.55 & 0.48 & 0.53 & 0.46 & 1.53 & 1.50 \\ 18 & 0.82 & 0.79 & 0.99 & 0.79 & 1.05 & 1.05 \\ 19 & 0.77 & 0.71 & 0.69 & 0.62 & & \\ 20 & 0.70 & 0.57 & 0.50 & 0.54 & 1.73 & 1.67 \\ 21 & 1.12 & 1.15 & 1.35 & 1.24 & 4.02 & 3.91 \\ 22 & 0.77 & 0.80 & 0.61 & 0.59 & 2.04 & 2.06 \\ 23 & 0.65 & 0.59 & 0.53 & 0.52 & 1.66 & 1.64 \\ 24 & 0.70 & 0.60 & 0.74 & 0.63 & 0.97 & 0.95 \\ 25 & 1.15 & 1.00 & 0.96 & 0.85 & 1.40 & 1.23 \\ 26 & 0.67 & 0.58 & 0.84 & 0.65 & 1.05 & 1.48 \\ 27 & 0.68 & 0.54 & 0.59 & 0.47 & 2.34 & 2.19 \\ 28 & 0.73 & 0.59 & 0.67 & 0.63 & 1.63 & 1.59 \\ 29 & 0.63 & 0.57 & 0.88 & 0.70 & 1.83 & 1.81 \\ 30 & 0.56 & 0.48 & 0.51 & 0.45 & & \\ 31 & 1.01 & 0.99 & 0.87 & 0.84 & 4.29 & 3.67 \\ 32 & 0.73 & 0.55 & 0.54 & 0.41 & 1.43 & 1.34 \\ 33 & 0.74 & 0.55 & 1.04 & 0.75 & 1.83 & 1.77 \\ 34 & 0.68 & 0.46 & 0.61 & 0.53 & 1.15 & 1.06 \\ 35 & 0.57 & 0.54 & 0.59 & 0.54 & 1.15 & 1.19 \\ 36 & 1.02 & 1.07 & 1.23 & 1.24 & 1.89 & 1.67\end{array}$




\section{Left Atrium ROI Arterial Input Function - Rest and Stress MBF}

\begin{tabular}{cccccc} 
Subject & Rest & \multicolumn{1}{l}{ Rest } & \multicolumn{1}{l}{ Rest } & \multicolumn{1}{l}{ Rest } & \multicolumn{1}{l}{ Stress } \\
& o6 1 & o6 soc 1 & \multicolumn{1}{l}{ o6 2 } & \multicolumn{1}{l}{ o6 soc 2 } & \multicolumn{1}{l}{ o6 soc } \\
1 & 0.61 & 0.60 & 0.69 & 0.69 & 2.58 \\
2 & 0.59 & 0.55 & 0.65 & 0.70 & 2.77 \\
3 & 0.59 & 0.57 & 0.55 & 0.52 & 1.42 \\
4 & 0.63 & 0.62 & 0.81 & 0.79 & 1.85 \\
5 & 1.44 & 1.37 & 1.38 & 1.27 & 2.04 \\
6 & 2.66 & 2.37 & 2.78 & 2.52 & 5.47 \\
7 & 0.68 & 0.66 & 0.62 & 0.62 & \\
8 & 0.84 & 0.80 & 1.19 & 1.16 & 2.26 \\
9 & 0.78 & 0.77 & 0.96 & 0.95 & \\
10 & 1.34 & 1.28 & 1.36 & 1.35 & 3.86 \\
11 & 0.47 & 0.47 & 0.46 & 0.46 & 0.94 \\
12 & 0.64 & 0.65 & 0.58 & 0.59 & 0.78 \\
13 & 0.76 & 0.75 & 0.77 & 0.77 & 1.61 \\
14 & 1.51 & 1.41 & 1.33 & 1.23 & 3.26 \\
15 & 1.30 & 1.29 & 1.17 & 1.20 & 2.07 \\
16 & 1.00 & 0.98 & 0.93 & 0.91 & 1.45 \\
17 & 0.44 & 0.44 & 0.46 & 0.46 & 1.19 \\
18 & 0.78 & 0.79 & 0.80 & 0.79 & 0.73 \\
19 & 1.09 & 1.03 & 0.94 & 0.87 & \\
20 & 0.61 & 0.60 & 0.53 & 0.50 & 1.94 \\
21 & 1.62 & 1.50 & 1.60 & 1.52 & 4.65 \\
22 & 0.74 & 0.74 & 0.74 & 0.73 & 1.68 \\
23 & 0.83 & 0.82 & 0.71 & 0.68 & 1.99 \\
24 & 0.63 & 0.63 & 0.63 & 0.62 & 1.00 \\
25 & 0.91 & 0.88 & 0.85 & 0.84 & 0.94 \\
26 & 0.72 & 0.77 & 0.88 & 0.76 & 1.17 \\
27 & 0.50 & 0.50 & 0.51 & 0.51 & 2.35 \\
28 & 0.51 & 0.51 & 0.54 & 0.55 & 1.86 \\
29 & 0.59 & 0.60 & 0.68 & 0.60 & 1.48 \\
30 & 0.46 & 0.46 & 0.45 & 0.44 & \\
31 & 1.08 & 1.06 & 1.22 & 1.15 & 3.07 \\
32 & 0.47 & 0.46 & 0.41 & 0.40 & 1.04 \\
33 & 0.55 & 0.55 & 0.60 & 0.60 & 1.34 \\
34 & 0.72 & 0.55 & 0.61 & 0.61 & 1.26 \\
35 & 0.53 & 0.57 & 0.56 & 0.60 & 1.17 \\
36 & 0.73 & 0.74 & 0.75 & 0.75 & 0.98
\end{tabular}




\section{Serial Subjects}

\section{Population Statistics}

\begin{tabular}{ccccccrrr} 
Subject & Gender & Age & Status & BMI & Rest & Stress & Rest & \multicolumn{1}{c}{ Stress } \\
& & & & & RPP 1 & RPP 1 & RPP 2 & RPP 2 \\
1 & m & 52 & CAD & 32.0 & 5141 & 7178 & 5805 & 7800 \\
2 & m & 69 & CAD & 33.2 & 8736 & 11325 & 7619 & 12008 \\
3 & m & 42 & & 32.8 & 5891 & 9900 & 6934 & 8944 \\
4 & m & 72 & CAD & 25.0 & 6235 & 7560 & 7018 & 8804 \\
5 & f & 65 & CAD & 20.0 & 7677 & 7695 & 6144 & 10419 \\
6 & m & 51 & CAD & 30.0 & 6077 & 7446 & 5565 & 7000 \\
7 & m & 54 & CAD & 25.0 & 5639 & 6264 & 6831 & 9401 \\
8 & m & 47 & CAD & 25.0 & 8514 & 14027 & 6698 & 10057 \\
9 & m & 56 & CAD & 40.0 & 5051 & 7420 & 4947 & 8184 \\
10 & m & 75 & CAD & 25.0 & 5483 & 9438 & 5429 & 6678 \\
11 & f & 72 & CAD & 26.0 & 8316 & 9108 & 9669 & 10010 \\
12 & m & 53 & CAD & 29.0 & 6993 & 9996 & 12012 & 13905 \\
13 & m & 47 & CAD & 34.0 & 4940 & 7107 & 5002 & 7519 \\
14 & f & 50 & & 20.4 & 6129 & 8475 & 5673 & 9794 \\
15 & m & 48 & CAD & 25.0 & 6105 & 12204 & 5985 & 10500 \\
16 & f & 72 & CAD & 25.0 & 6683 & 8175 & 6797 & 8463 \\
17 & f & 63 & CAD & 34.9 & 8651 & 11232 & 6514 & 6970 \\
18 & m & 74 & CAD & 33.0 & 8543 & 10586 & 9932 & 11160 \\
19 & f & 71 & CAD & 31.0 & 6826 & 11424 & 6947 & 8856 \\
20 & f & 68 & CAD & 33.7 & 7735 & 11544 & 8482 & 10425 \\
21 & m & 65 & CAD & 28.7 & 5389 & 8632 & 7293 & 10384 \\
22 & m & 76 & CAD & 31.9 & 7784 & 11097 & 6900 & 9300 \\
23 & m & 34 & CAD & 19.0 & 6392 & 9984 & 5735 & 8463 \\
24 & m & 75 & CAD & 24.0 & 9621 & 10738 & 11653 & 12212 \\
25 & m & 61 & CAD & 35.0 & 5738 & 7056 & 5076 & 6900 \\
26 & m & 56 & CAD & 25.0 & 7051 & 7828 & 5513 & 6512 \\
27 & m & 53 & CAD & 27.7 & 6030 & 6204 & 6180 & 6588 \\
28 & f & 42 & CAD & 27.0 & 6592 & 8670 & 5311 & 7760 \\
29 & m & 65 & CAD & 31.0 & 6953 & 10507 & 6250 & 9450 \\
30 & f & 50 & & 19.5 & 8836 & 9234 & 6555 & 8970 \\
31 & m & 66 & CAD & 27.0 & 7493 & 8866 & 9599 & 9860 \\
32 & f & 80 & CAD & 31.2 & 8085 & 8888 & 10769 & 8544 \\
& & & & & & & & \\
& & & & &
\end{tabular}




\section{Rest and Stress MBF}

$\begin{array}{ccccc}\text { Subject } & \text { Rest } & \text { Rest } & \text { Stress } & \begin{array}{c}\text { Stress } \\ \end{array} \\ \text { MBF } & \text { MBF } & \text { MBF } & \text { MBF } \\ 1 & 0.48 & 0.40 & 2.10 & 1.84 \\ 2 & 0.80 & 0.56 & 2.30 & 2.11 \\ 3 & 0.56 & 0.69 & 2.16 & 2.79 \\ 4 & 0.58 & 0.64 & 1.45 & 1.26 \\ 5 & 0.84 & 0.75 & 1.83 & 2.44 \\ 6 & 0.47 & 0.49 & 0.96 & 0.72 \\ 7 & 0.42 & 0.43 & 1.74 & 1.42 \\ 8 & 1.27 & 0.82 & 4.46 & 1.85 \\ 9 & 0.43 & 0.41 & 1.40 & 1.15 \\ 10 & 0.47 & 0.64 & 1.47 & 0.70 \\ 11 & 1.10 & 0.76 & 2.38 & 1.30 \\ 12 & 0.55 & 0.68 & 0.94 & 1.39 \\ 13 & 0.39 & 0.28 & 1.27 & 1.07 \\ 14 & 0.78 & 0.89 & 2.38 & 3.50 \\ 15 & 0.57 & 0.53 & 0.66 & 0.70 \\ 16 & 0.71 & 0.89 & 1.38 & 2.45 \\ 17 & 1.23 & 0.66 & 3.09 & 1.75 \\ 18 & 0.50 & 0.77 & 0.92 & 1.40 \\ 19 & 0.97 & 1.06 & 1.49 & 1.60 \\ 20 & 0.70 & 0.84 & 2.15 & 2.29 \\ 21 & 0.60 & 0.60 & 2.02 & 2.20 \\ 22 & 0.46 & 0.40 & 0.99 & 1.05 \\ 23 & 1.12 & 0.88 & 3.28 & 3.12 \\ 24 & 0.96 & 1.05 & 1.81 & 1.86 \\ 25 & 0.35 & 0.36 & 0.49 & 0.63 \\ 26 & 0.51 & 0.45 & 1.31 & 0.98 \\ 27 & 0.58 & 0.58 & 1.19 & 1.16 \\ 28 & 1.12 & 0.90 & 2.40 & 1.99 \\ 29 & 0.51 & 0.46 & 1.40 & 1.26 \\ 30 & 1.58 & 1.37 & 4.67 & 3.01 \\ 31 & 0.61 & 0.84 & 0.93 & 1.84 \\ 32 & 0.75 & 0.87 & 1.10 & 0.92\end{array}$




\section{References}

1. Efseaff, M., Klein, R., Ziadi, M., Beanlands, R., \& deKemp, R. Short-term repeatability of resting myocardial blood flow measurements using rubidium-82 PET imaging. I Nucl Cardiol. 2012 [in press].

2. Efseaff, M., Klein, R., Beanlands, R., \& deKemp, R. Short-term repeatability of absolute myocardial blood flow using rubidium-82 PET. J Nucl Med. 2012, 53 (Supplement 1):1768. (abstract)

3. Heart \& Stroke Foundation of Canada. Statistics. [Online] 2012. [Cited: June 18, 2012.] http://www.heartandstroke.com/site/c.iklQLCMWJtE/b.3483991/k.34A8/Statistics.htm.

4. National Heart Lung and Blood Institute. What is Coronary Heart Disease? [Online] 2012. [Cited: June 18, 2012.] http://www.nhlbi.nih.gov/health/health-topics/topics/cad/.

5. Cerqueira, M., Weissman, N., Dilsizian, V., Jacobs, A., Kaul, S., Laskey, W., Warren, K., Pennel, D., Rumberger, J. Ryan, T., \& Verani, M. Standardized Myocardial Segmentation and Nomenclature for Tomographic Imaging of the Heart: A Statement for Healthcare Professionals From the Cardiac Imaging Committee of the Council on Clinical Cardiology of the American Heart Association. Circulation. 2002, Issue 105, pp. 539-542.

6. American Society of Nuclear Cardiology. Pharmacologic and Exercise Stress Tests [Online] 2012. [Cited: Sept. 15, 2012] http://www.asnc.org/media/PDFs/PPStressTests081511.pdf

7. Czernin, J., Muller, P., Chan, S., Bunken, R., Porenta, G., Krivokapich, J., Chen, K., Chan, A., Phelps, M., \& Schelbert, H. Influence of Age and Hemodynamics on Myocardial Blood Flow and Flow Reserve. Circulation. 1993, Issue 88, pp. 62-69.

8. Levin, C. Basic Physics of Radionuclide Imaging. In Emission Tomography: The Fundamentals of PET and SPECT. M. Wernick \& J. Aarsvold [Eds]. San Diego : Elsevier Academic Press, 2004, pp. 53-88.

9. Cherry, S., Sorensen, J., \& Phelps, M. Physics in Nuclear Medicine. 3rd edition. Philadelphia : Saunders, 2003, pp. 325-61.

10. Baily, D., Karp, J., \& Surti, S. Physics and Instrumentation in PET. In Positron Emission Tomography. D. Baily, D. Townsend, P. Valk, \& M. Maisey [Eds]. Singapore : Springer-Verlag, 2005, pp. 14-40.

11. Wilkinson III, F. Scintillators. In Emission Tomography: The Fundamentals of PET and SPECT. M. Wernick \& J. Aarsvold [Eds]. San Diego : Elsevier Academic Press, 2004, pp. 229-253. 
12. Turkington, T. PET Physics and PET Instrumentation. In Principles and Practice of PET and PET/CT. R. Wahl [Ed]. $2^{\text {nd }}$ edition. Philadelphia : Lippincot Williams \& Wilkins, 2009, pp. 47-57.

13. Di Carli, M., \& Lipton, M. Cardiac PET and PET/CT Imaging. New York: Springer Science + Business Media, LLC, 2007, pp. 3-18.

14. Casey, M., Gadagkar, H., \& Newport, D. A component based method for normalization in volume PET. Procedings of the 3rd International Meeting on Fully Three-Dimensional Reconstruction in Radiology and Nuclear Medicine. 1995, pp. 67-71.

15. Lewellen, T. \& Karp, J. PET systems. In Emission Tomography: The Fundamentals of PET and SPECT. M. Wernick \& J. Aarsvold [Eds]. San Diego : Elsevier Academic Press, 2004, pp. 179-192.

16. Meikle, S., \& Badawi, R. Quantitative Techniques in PET. In Positron Emission Tomography. D. Baily, D. Townsend, P. Valk, \& M. Maisey [Eds]. Singapore : Springer-Verlag London Limited, 2005, pp. 93-126.

17. Watson, C., Hayden, C., Casey, M., Hamill, J., \& Bendriem, B. Prompt gamma correction for improved quantification in 82-Rb PET. I Nucl Med. 2008, 49 (Supplement 1), p. 64. (abstract)

18. Watson, C., Townsend, D., \& Bendriem, B. PET/CT Systems. In Emission Tomography: The Fundamentals of PET and SPECT. M. Wernick \& J. Aarsvold [Eds]. San Diego: Elsevier Inc, 2004, pp. 195-212.

19. Cherry, S., Sorenson, J., \& Phelps, M. Physics in Nuclear Medicine. 3rd edition. Philadelphia : Saunders, 2003, pp. 165-182.

20. Cherry, S., Sorenson, J., \& Phelps, M. Physics in Nuclear Medicine. 3rd edition. Philadelphia : Saunders, 2003, pp. 273-298.

21. GE Healthecare. Discovery PET/CT 690 Data Sheet. Mississauga, Ontario: General Electric Company, 2010.

22. Bateman, T., Heller, G., McGhie, I., Friedman, J., Case, J., Bryngelson, J., Hertenstein, G., Moutray, K., Reid, K., Cullom, S. Diagnostic accuray of rest/stress ECG gated Rb-82 myocardial perfusion PET; Comparison with ECG-gated Tc-99m sestamibi SPECT. J Nucl Cardiol. 2006, 13, pp. 24-33.

23. Epstein, N., Benelfassi, A., Beanlands, R., \& deKemp, R. A 82Rb infusion system for quantitative perfusion imaging with 3D PET. Applied Radiation and Isotopes. 2004, 60, pp. 921 927.

24. Ziadi, M., \& Beanlands, R. The clinical utility of assessing myocardial blood flow using positron emission tomography. I Nucl Cardiol. 2010, 17, pp. 571-581. 
25. Ziadi, M., deKemp, R., Williams, K., Guo, A., Renaud, J., \& Chow, B. Does Quantification of Myocardial Flow Reserve Using Rubidium-82 Positron Emission Tomography Facilitate Detection of Multivessel C6ronary Artery Disease? J Nucl Cardiol. 2012, 19(4), pp. 670-680.

26. Parkash, R., deKemp, R., Ruddy, T., Kitsikis, A., Hart, R., \& Beauchesne, L. Potential utility of rubidium 82 PET quantification in patients with 3-vessel coronary artery disease. J Nucl Cardiol. 2004,11 , pp. 440-449.

27. Ziadi, M., deKemp, R., Williams, K., Guo, A., Chow, B., \& Renaud, J. Imparied Myocardial Flow Reserve on Rubidium-82 Positron Emission Tomography Imaging Predicts Adverse Outcomes in Patients Assessed for Myocardial Ischemia. J Am Coll Cardiol. 2011, 58, pp. 740-748.

28. Kajander, S., Joutsiniemi, E., Saraste, M., Pietilaa, M., Ukkonen, H., Saraste, A., Sipila, H., Teraas, M., Maaki, M., Airaksninen, J., Hartiala, J., Knuuti, J. Clinical Value of Absolute Quantification of Myocardial Perfusion with ${ }^{15} \mathrm{O}$-water in Coronary Artery Disease. Circulation. 2011, 4, pp. 678-684.

29. Yoshinaga, K., Katoh, C., Manabe, O., Klein, R., Naya, M., Sakakibara, M., Yamada, S., deKemp, R., Tsutsui, H., Tamaki, N. Incremental Diagnostic Value of Regional Myocardial Blood Flow Quantification Over Relative Perfusion Imaging With Generator-Produced Rubidium-82 PET. Circulation Journal. 2011, 75, pp. 2628-2634.

30. El Fakhri, G., Kardan, A., Sitek, A., Dorbala, S., Abi-Hatem, N., Lahoud, Y., Fischman, A., Coughlan, M., Yasuda, T., Di Carli, M. Reproducibility and accuracy of quantitative myocardial blood flow assessment with ${ }^{82} \mathrm{Rb}$ PET: Comparison with ${ }^{13} \mathrm{~N}$-ammonia PET. I NuCl Med. 2009, 50 , pp. 1062-1071.

31. Lautamaaki, R., George, R., Kitagawa, K., Higuchi, T., Merrill, J., \& Voicu, C. Rubidium-82 PET$\mathrm{CT}$ for quantitative assessment of myocardial blood flow: validation in a canine model of coronary artery stenosis. Eur J Nucl Med Mol Imaging. 2009, 36, pp. 576-586.

32. Muzik, O., Duvernoy, C., Beanlands, R., Sawada, S., Dayanikli, F., Wolfe Jr., E., Schwaiger, M. Assessment of Diagnostic Performance of Quantitative Flow Measurements in Normal Subjects and Patients with Angiographically Documented Coronary Artery Disease by Means of Nitrogen13 Ammonia and Positron Emission Tomography. J Am Coll Cardiol. 1998, 31, pp. 534-540.

33. Koeppe, R. Data Analysis and Image Processing. In Principles and Practice of PET and PET/CT. R. Wahl [Ed]. 2nd edition. Philadelphia: Lippincott Williams \& Wilkins, 2009, pp. 69-105.

34. Renkin, E. Transport of potassium-42 from blood to tissue isolated mammalian skeletal muscles. Am J Physiol. 1959, 197, pp. 1205-1210. 
35. Crone, $C$. Permeability of capillaries in various organs as determined by use of the indicator diffusion method. Acta Physiol Scand. 1963, 8, pp. 292-305.

36. Schelbert, HR. In Cardiac Imaging: A Companion to Braunwald's Heart Disease. M. Marcus, H. Schelbert, D. Skorton, \& G. Wolf [Eds]. Philadelphia: W. B. Saunders Company, 1991.

37. Golanoski, L., deKemp, R., Beanlands, R., \& Ruddy, T. Variance and covariance of $82 R b$ kinetic parameters: Computer simulations and dynamic PET studies. Proceedings of the 22nd Annual International Conference of the IEEE EMBS. 2000, Vol. 2, pp. 1096-1099.

38. Bergmann, S., Fox, K., Rand, A., McElvany, K., Welch, M., \& Markham, J. Quantification of regional myocardial blood flow in vivo with $\mathrm{H}_{2}{ }^{15} \mathrm{O}$. Circulation. 1984, 70, pp. 724-733.

39. DeGrado, T., Hanson, M., Turkington, T., Delong, D., Brezinski, D., \& Vallee, J. Estimation of myocardial blood flow for longitudinal sudies with ${ }^{13} \mathrm{~N}$-labeled ammonia and positron emission tomography. J Nucl Cardiol. 1996, 3, pp. 494-507.

40. Finn, R., \& Schlyer, D. Production of Radionuclides for PET. In Principles and Practice of PET and PET/CT. R. Wahl [Ed]. 2nd edition. Philadelphia: Lippincott Williams \& Wilkins, 2009, pp. 115.

41. Thompson, C. In Principles and Practice of Positron Emission Tomography. J. Buchanan [Ed]. Philadelphia : Lippincott Williams \& Wilkins, 2002, pp. 48-64.

42. deKemp, R., Yoshinaga, K., \& Beanlands, R. Will 3-Dimensional PET-CT Enable the Routine Quantification of Myocardial Blood Flow? J Nucl Cardiol. 2007, 14, pp. 380-397.

43. Klein, R., Beanlands, R., \& deKemp, R. Review Article--Quantification of myocardial blood flow and flow reserve: Technical aspects. Journal of Nuclear Cardiology. 2010, 17, pp. 555-570.

44. Hall, J., \& Guyton, A. Textbook of medical physiology. St. Louis: Elsevier Saunders, 2006. 07216-0240-1.

45. Nestrov, S., Han, C., Maki, M., Kajander, S., Naum, A., Helenius, H., Lisinen, I., Ukkonen, H., Pietilaa, M., Joutsiniemi, E., Knuuti, J. Myocardial perfusion quantification with ${ }^{15} \mathrm{O}$-labeled water PET: High reproducibility of the new cardiac analysis software Carimas (TM). Eur J NuCl Med Mol Imaging. 2009, 36, pp. 1594-1602.

46. PMOD Technologies. [Online] http://www.pmod.com. [Cited: June 18, 2012]

47. Cedars-Sinai. Quantitative PET (QPET). [Online] http://www.cedarssinai.edu/Patients/Programs-and-Services/Medicine-Department/Artificial-Intelligence-inMedicine-AIM/Projects/Quantitative-PET-QPET.aspx. [Cited: June 18, 2012] 
48. Slomka, P., Alexanderson, E., Jacome, R., Jimenez, M., Romero, E., Meave, A., Le Meunier, L., Dalhbom, M., Berman, D., Germano, G., Schelbert, H. Comparison of Clinical Tools for Measurements of Regional Stress and Rest Myocardial Blood Flow Assessed with ${ }^{13} \mathrm{~N}$-Ammonia PET/CT. The Journal of Nuclear Medicine. 2012, Vol. 53, 2, pp. 171-181.

49. Sunderland, J. Comparison of Rb-82-based quantitative myocardial blood flow measurements across three analysis platforms. J Nucl Med. 2012, 53 (Supplement 1):1799. (abstract)

50. Klein, R., Renaud, J., Ziadi, M., Thorn, S., Adler, A., Beanlands, R., deKemp, R. Intra- and interoperator repeatability of myocardial blood flow and myocardial flow reserve measurements using rubidium-82 PET and a highly automated analysis system. J Nucl Cardiol. 2010, 17(4), pp. 600-616.

51. Schindler, T., Zhang, X., Prior, J., Dadenas, J., Dahlbom, M., \& Sayre, J. Assessment of intraand interobserver reproducibility of rest and cold pressor test-stimulated myocardial blood flow with ${ }^{13} \mathrm{~N}$-ammonia and PET. Eur J Nucl Med Mol Imaging. 2007, 34, pp. 1178-1188.

52. Nagamachi, S., Czernin, J., Kim, A., Sun, K., Bottcher, M., Phelps, M., \& Schelbert, H. Reproducibility of Measurements of Regional Resting and Hyperemic Myocardial Blood Flow Assessed with PET. J Nucl Med. 1996, 37(10), pp. 1626-1631.

53. Sawada, S., Muzik, O., Beanlands, R., Wolfe, E., Hutchins, G., \& Schwaiger, M. Interobserver and interstudy variability of myocardial blood flow and flow reserve measurements with nitrogen 13 ammonia-labeled positron emission tomography. J Nucl Cardiol. 1995, 2(5), pp. $413-$ 422.

54. Jagathesan, R., Kaufmann, P., Rosen, S., Rimoldi, O., Turkeimer, F., \& Foale, R. Assessment of the Long-Term Reproducibility of Baseline and Dobutamine-Induced Myocardial Blood Flow in Patients with Stable Coronary Artery Disease. J Nucl Med. 2005, 46, pp. 212-219.

55. Kaufmann, P., Gnecchi-Ruscone, T., Yap, J., Rimoldi, O., \& Camici, P. Assessment of the reproducibility of baseline and hyperemic myocardial blood flow measurements with ${ }^{15} \mathrm{O}$ labeled water and PET. I Nucl Med. 1999, 40, pp. 1848-1856.

56. Wyss, C., Koepfli, P., Mikolajczyk, K., Burger, C., von Schulthess, G., \& Kaufmann, P. Bicycle Exercise Stress in PET for Assessment of Coronary Flow Reserve: Repeatability and Comparison with Adenosine Stress. J Nucl Med. 2003, 44, pp. 146-154.

57. Manabe, O., Yoshinaga, K., Katoh, C., Naya, M., deKemp, R., \& Tamaki, N. Repeatability of Rest and Hyperemic Myocardial Blood Flow Measurements with ${ }^{82} \mathrm{Rb}$ Dynamic PET. J Nucl Med. 2009, 50, pp. 68-71. 
58. deKemp, R., Klein, R., Lortie, M., \& Beanlands, R. Constant-Activity-Rate Infusions for Myocardial Blood Flow Quantification with ${ }^{82} \mathrm{Rb}$ and $3 \mathrm{D}$ PET. IEEE Nuclear Science Symposium Conference Record. 2006, Vol. 6, pp. 3519-3521. [abstract]

59. Klein, R., Adler, A., Beanlands, R., \& deKemp, R. Precision-controlled elution of a ${ }^{82} \mathrm{Sr} /{ }^{82} \mathrm{Rb}$ generator for cardiac perfusion imaging with positron emission tomography. Phys Med Biol. 52, 2007, pp. 659-673.

60. Ruby-Fill (TM) Product Monograph. Rubidium (82Rb) Generator Diagnostic Radiopharmaceutical (Myocardial Imaging). Jubilant DraxImage: Kirkland, QC. [Cited: June, 2012].

61. Hove, J., lida, H., Kofoed, K., Freiburg, J., Holm, S., \& Kelbaek, H. Left atrial versus left ventricular input function for quantification of the myocardial blood flow with nitrogen-13 ammonia and positron emission tomography. Eur J Nucl Med Mol Imaging. 2004, 31, pp. 71-76.

62. Katoh, C., Morita, K., Shiga, T., Kubo, N., Nakada, K., \& Tamaki, N. Improvement of Algorithm for Quantification of Regional Myocardial Blood Flow Using ${ }^{15} \mathrm{O}-$ Water with PET. J Nucl Med. 2004, Vol. 45, 11, pp. 1908-1916.

63. Altman, D. \& Bland, J. Measurement in medicine: the analysis of method comparison studies. Statistitian. 1983, 32, pp. 307-317.

64. Lortie, M., Beanlands, R., Yoshinaga, K., Klein, R., DaSilva, J., \& deKemp, R. Quantification of myocardial blood flow with ${ }^{82} \mathrm{Rb}$ dynamic PET imaging. Eur J Nucl Med Mol Imaging. 2007, 34 , pp. 1765-1774.

65. Klein, R., Katoh, C., Yoshinaga, K., Tamaki, N., Adler, A., Beanlands, R., deKemp, R. Evaluation of Myocardium to Blood Pool Spillover Correction in Quantification Myocardial Blood Flow with 82Rb PET. Montreal, Quebec, Canada: World Molecular Imaging Congress, September 24, 2009. [abstract]

66. Hutchins, G., Caraher, J., \& Raylman, R. A Region of Interest Strategy for Minimizing Resolution Distotions in Quantitative Myocardial PET Studies. I Nucl Med. 1992, 3, pp. $1243-$ 1250.

67. Madsen, M. A simplified formulation of the gamma variate function. Phys Med Biol. 1992, Vol. 37, 7, pp. 1597-1600.

68. Thompson, H., Starmer, C., Whalen, R., \& McIntosh, H. Inticator Transit Time Considered as a Gamma Variate. Circulation Research. 1964, 14, pp. 502-515. 
69. Sdringola, S., Johnson, N., Kirkeeide, R., Cid, E., \& Gould, L. Impact of Unexpected Factors on Quantitative Myocardial Perfusion and Coronary Flow Reserve in Young, Asymptomatic Volunteers. JACC: Cardiovascular Imaging, 4(4), 2011, pp. 402-412.

70. Bouchareb, Y., Thielemans, K., Spinks, T., Rimoldi, O., \& Camici, P. Comparison of analytic and iterative reconstruction methods for quantitative cardiac PET studies in 3D using Oxygen-15 water scans. IEEE Nuclear Science Symposium Conference Record. 2005, Vol. 4, pp. 2120-2123.

71. Chen, G., Branch, K., Alessio, A., Pham, P., Tabibiazar, R., Kinahan, P., Caldwell, J. Effect of Reconstruction Algorithms on Myocardial Blood Flow Measurement with ${ }^{13} \mathrm{~N}$-Ammonia PET. J Nucl Med. 2007, 48, pp. 1259-1265.

72. Hove, J., Rasmussen, R., Freiberg, J., Holm, S., Kelbaek, H., \& Kofoed, K. Clinical evaluation of iterative reconstruction (ordered-subset expectation maximization) in dynamic positron emission tomography: Quantitative effects on kinetic modeling with $\mathrm{N}-13$ ammonia in healthy subjects. J Nucl Cardiol. 2008, Vol. 15, 4, pp. 530-534.

73. Boellaard, R., van Lingen, A., \& Lammertsma, A. Experimental and Clinical Evaluation of Iterative Reconstruction (OSEM) in Dynamic PET: Quantitative Characteristics and Effects on Kinetic Modeling. J Nucl Med. 2001, 48, pp. 808-817.

74. Lubberink, M., Boellaard, R., van der Weerdt, A., Visser, F., \& Lammertsma, A. Quantitative Comparison of Analytic and Iterative Reconstruction Methods in 2- and 3-Dimensional Dynamic Cardiac ${ }^{18}$ F-FDG PET. J Nucl Med. 2004, Vol. 45, 12, pp. 2008-2015.

75. Katoh, C., Yoshinaga, K., Klein, R., Kasi, K., Tomiyama, Y., Manabe, O., Quantification of regional myocardial blood flow estimation with three-dimensional dynamic rubidium-82 PET and modified spillover correction model. J Nucl Cardiol. 2012, 19(4), pp. 763-774.

76. Heyndrickx, G., Millard, R., McRitchie, R., Maroko, P., \& Vatner, S. Regional myocardial functional and electrophysical alterations after brief coronary artery occlusion in dogs. $J$ Clin Invest. 1975, 56(4), pp. 978-985.

77. Lamoureux, M., Thorn, S., Dumouchel, T., Renaud, J., Klein, R., Mason, S., Lortie, M., Dasilva, J., Beanlands, R., deKemp, R. Uniformity and repeatability of normal resting myocardial blood flow in rats using ${ }^{13} \mathrm{~N}$-ammonia and small animal PET. Nucl Med Commun. June 10, 2012. [Epub ahead of print].

78. Murthy, V., Masanao, N., Foster, C., Hainer, J., Gaber, M., Di Carli, G., Blankstein, R., Dorbala, S., Sitek, A., Pencina, M., Di Carli, M. Improved Cardiac Risk Assessment With Noninvasive Measures of Coronary Flow Reserve. Circulation. 2011, 124, pp. 2215-2224. 
79. deKemp, R., Slomka, P., Declerck, J., Pan, X., Klein, R., Nakazato, R., Germano, G., Tonge, C., Beanlands, R., Berman, D. Reproducible imaging of myocardial flow reserve with rubidium-82 PET - a multicentre multi-vendor comparison study. European Heart J. 2011, 13 (suppl): A76 (abstract)

80. Gould, K., Goldstein, R., Mullani, N., Kirkeeide, R., Wong, W., Tewson, T., Berridge, M., Bolomey, L., Hartz, R., Smalling, R., Fuentes, F., Nishikawa, A. Noninvasive Assessment of Coronary Stenosis by Myocardial Perfusion Imaging During Pharmacological Coronary Vasodilation. VII. Clinical Feasibility of Positron Cardiac Imaging Without a Cyclotron Using Generator-Produced Rubidium-82. J Amer Coll Cardiol. 1986, Vol. 7, 4, pp. 775-789.

81. Massart, D., Smeyers-Verbeke, J., Capron, X., \& Schlesler, K. Practical Data Handling: Visual Presentation of Data by Means of Box Plots. LCGC Europe. 2005, 18(4), pp. 215-218. 